SOLUTION OF THE ANTENNA PLACEMENT PROBLEM

BY MEANS OF GLOBAL OPTIMIZATION TECHNIQUES

A THESIS SUBMITTED TO

THE GRADUATE SCHOOL OF NATURAL AND APPLIED SCIENCES

OF

MIDDLE EAST TECHNICAL UNIVERSITY

BY

MUSTAFA URAL

IN PARTIAL FULFILLMENT OF THE REQUIREMENTS

FOR

THE DEGREE OF MASTER OF SCIENCE

IN

ELECTRICAL AND ELECTRONICS ENGINEERING

OCTOBER 2010 
Approval of the thesis:

\section{SOLUTION OF THE ANTENNA PLACEMENT PROBLEM BY MEANS OF GLOBAL OPTIMIZATION TECHNIQUES}

submitted by MUSTAFA URAL in partial fulfillment of the requirements for the degree of Master of Science in Electrical and Electronics Engineering Department, Middle East Technical University by,

Prof. Dr. Canan Özgen

Dean, Graduate School of Natural and Applied Sciences

Prof. Dr. İsmet Erkmen

Head of Department, Electrical and Electronics Engineering

Prof. Dr. Mustafa Kuzuoğlu

Supervisor, Electrical and Electronics Engineering Dept., METU

\section{Examining Committee Members:}

Prof. Dr. Gönül Turhan Sayan

Electrical and Electronics Engineering Dept., METU

Prof. Dr. Mustafa Kuzuoğlu

Electrical and Electronics Engineering Dept., METU

Prof. Dr. Gülbin Dural

Electrical and Electronics Engineering Dept., METU

Assist. Prof. Dr. Lale Hayırlığlu Alatan

Electrical and Electronics Engineering Dept., METU

Assist. Prof. Dr. Asım Egemen Yılmaz

Electronics Engineering Dept., Ankara University

Date: $25 / 10 / 2010$ 
I hereby declare that all information in this document has been obtained and presented in accordance with academic rules and ethical conduct. I also declare that, as required by these rules and conduct, I have fully cited and referenced all material and results that are not original to this work.

Name, Last name : MUSTAFA URAL

Signature 


\begin{abstract}
SOLUTION OF THE ANTENNA PLACEMENT PROBLEM BY MEANS OF GLOBAL OPTIMIZATION TECHNIQUES

\author{
Ural, Mustafa \\ M.Sc., Department of Electrical and Electronics Engineering \\ Supervisor: Prof. Dr. Mustafa Kuzuoğlu
}

October 2010, 113 pages

In this thesis work, minimization of platform-based coupling between the antennas of two VHF radios on an aircraft platform and two HF radios on a ship platform is aimed. For this purpose; an optimal antenna placement, which yields minimum average coupling between the antennas over the whole frequency band of operation is determined for each platform. Two important global optimization techniques, namely Genetic Algorithm Optimization and Particle Swarm Optimization, are used in determination of these optimal antenna placements. Aircraft \& ship platforms and antennas placed on them are modeled based on their real electrical and physical properties in CST - MWS (Microwave Studio) simulation tool. For each platform, antenna placements and coupling results determined by two different optimization techniques and performances of these optimization techniques are compared with each other. At the end of this thesis work; for each platform, far-field radiation pattern performances of the antennas at their optimal places are analyzed in terms of directivity and coverage.

Keywords: Genetic Algorithm Optimization, Particle Swarm Optimization, Antenna Placement Analysis 


\title{
ÖZ
}

\section{ANTEN YERLEŞTIRME PROBLEMININ TÜMEL OPTIMMIZASYON YÖNTEMLERİ İLE ÇÖZÜMLENMESİ}

\author{
Ural, Mustafa \\ Yüksek Lisans, Elektrik ve Elektronik Mühendisliği Bölümü \\ Tez Yöneticisi: Prof. Dr. Mustafa Kuzuoğlu
}

Ekim 2010, 113 sayfa

$\mathrm{Bu}$ çalışmada, uçak platformuna yerleştirilecek iki VHF telsizin ve gemi platformuna yerleştirilecek iki HF telsizin antenlerinin, platform bazında birbirleri arasındaki bağlaşımının en aza indirgenmesi amaçlanmıştır. Bu amaçla, her iki platform için, antenler arası bağlaşım seviyelerinin çalışma bantları boyunca alınan ortalamalarının en az olduğu anten yerleşimi tespit edilmiştir. Bu optimal yerleşimin tespitinde, iki önemli tümel optimizasyon yöntemi olan Genetik Algoritma Optimizasyonu ve Parçacık Sürüsü Optimizasyonu kullanılmıştır. Uçak, gemi platformları ve bu platformlara ait antenler, platformların ve antenlerin gerçek fiziksel ve elektriksel özellikleri temel alınarak CST - MWS (Microwave Studio) simülasyon aracında modellenmiştir. Her iki platform için farklı optimizasyon yöntemleri kullanılarak tespit edilen anten yerleşimleri, bağlaşım sonuçları ve bu iki optimizasyon yönteminin performansları birbirleri ile karşılaştırılmıştır. En nihayetinde, her iki platform için de ortalama bağlaşımın en az olduğu anten yerleşiminde, antenlerin uzak alan 1şıma örüntüsü, kapsama ve yönlülük değerleri açısından incelenmiştir.

Anahtar Kelimeler: Genetik Algoritma Optimizasyonu, Parçacık Sürüsü Optimizasyonu, Anten Yerleşim Analizi 
To My Lonely and Beautiful Country 


\section{ACKNOWLEDGEMENTS}

I would like to express my greatest gratitude to my supervisor Prof. Dr. Mustafa Kuzuoğlu for his guidance, support, insight and credence throughout this thesis work.

I appreciate Can Bayseferoğulları for his ideas and contributions to this thesis work and I would like to thank Gönenç Afacan for spending his time for this thesis work.

I would like to acknowledge ASELSAN Inc. for providing the necessary platform for my research and computational activities.

I would also like to thank TÜBİTAK for supporting financially this thesis work.

My deepest and dearest gratitude goes to my mother Emriye Ural, my father Ahmet Ural and Buket Özen for their endless encouragement and love. 


\section{TABLE OF CONTENTS}

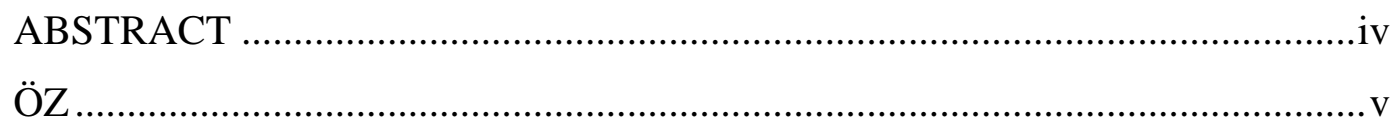

ACKNOWLEDGEMENTS ...................................................................................

TABLE OF CONTENTS .......................................................................... viii

LIST OF TABLES ....................................................................................

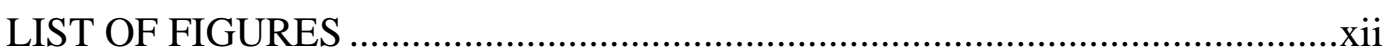

\section{CHAPTERS}

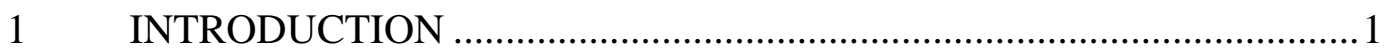

2 GLOBAL OPTIMIZATION TECHNIQUES: GENETIC ALGORITHM OPTIMIZATION AND PARTICLE SWARM OPTIMIZATION .........................6

$2.1 \quad$ Genetic Algorithm Optimization ...............................................10

2.1.1 Theory of Operation ........................................................ 11

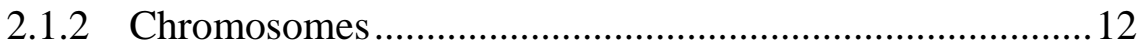

2.1.3 Encoding of Chromosomes (Phenotype-to-Genotype Encoding) .......................................................................... 16

2.1.4 Initial Population ..........................................................19

2.1.5 Selection, Crossover and Mutation ...................................20

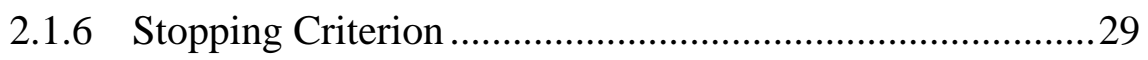

$2.2 \quad$ Particle Swarm Optimization......................................................29

2.2.1 Theory of Operation ........................................................... 30

2.2.2 Particles, Velocity \& Position Vectors................................32

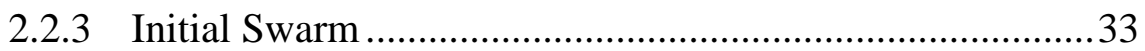

2.2.4 Updating velocity \& position vectors.................................34

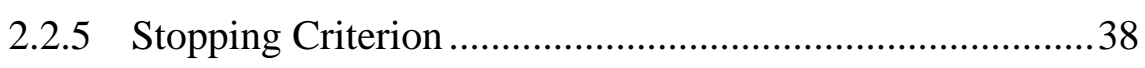


4 MODELING OF AIRCRAFT, SHIP AND THEIR ANTENNAS .............41

4.1 Modeling of Aircraft Platform and Its Antennas ...........................41

4.2 Modeling of Ship Platform and Its Antennas ..............................44

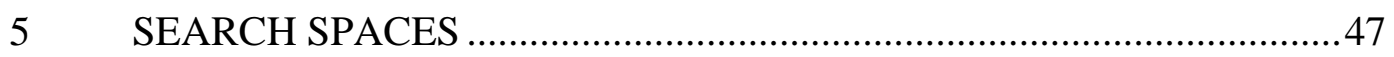

5.1 Search Spaces of VHF Antennas on Aircraft Platform .................47

5.2 Search Spaces of HF Antennas on Aircraft Platform .....................51

6 OPTIMIZATION OF ANTENNA PLACEMENTS …............................52

6.1 Optimization of Antenna Placement on Aircraft ............................54

6.1.1 Optimization of Antenna Placement on Aircraft by Genetic Algorithm Optimization ...................................................54

6.1.2 Optimization of Antenna Placement on Aircraft by Particle Swarm Optimization.........................................................62

6.2 Optimization of Antenna Placement on Ship ..............................66

6.2.1 Optimization of Antenna Placement on Ship by Genetic Algorithm Optimization

6.2.2 Optimization of Antenna Placement on Ship by Particle

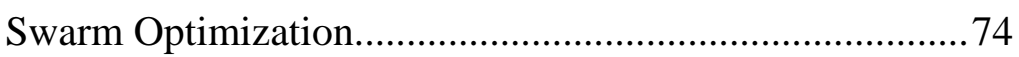

7 FAR-FIELD RADIATION PATTERN PERFORMANCES OF

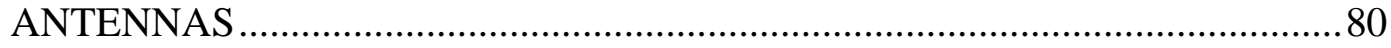

7.1 Far-field Radiation Pattern Performances of Aircraft Antennas ...81

7.1.1 Far-field Radiation Pattern Analyses for Aircraft Antennas at $30 \mathrm{MHz}$

7.1.2 Far-field Radiation Pattern Analyses for Aircraft Antennas at $40 \mathrm{MHz}$

7.1.3 Far-field Radiation Pattern Analyses for Aircraft Antennas at $50 \mathrm{MHz}$

7.1.4 Far-field Radiation Pattern Analyses for Aircraft Antennas at $60 \mathrm{MHz}$ 
7.1.5 Far-field Radiation Pattern Analyses for Aircraft Antennas

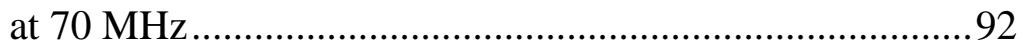

7.1.6 Far-field Radiation Pattern Analyses for Aircraft Antennas

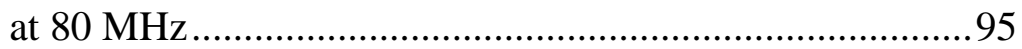

7.2 Far-field Radiation Pattern Performances of Ship Antennas.........98

7.2.1 Far-field Radiation Pattern Analyses for Ship Antennas at $10 \mathrm{MHz}$ .99

7.2.2 Far-field Radiation Pattern Analyses for Ship Antennas at $20 \mathrm{MHz}$ 101

7.2.3 Far-field Radiation Pattern Analyses for Ship Antennas at

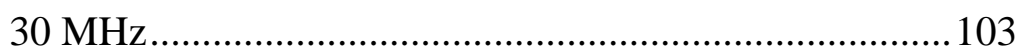

8 CONCLUSIONS AND FUTURE WORK ............................................ 107

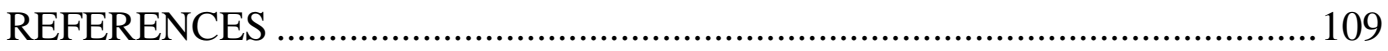




\section{LIST OF TABLES}

\section{TABLES}

Table 2.1 Phenotype-to-genotype encoding of $f(x, y)$......................................18

Table 6.1 Antenna placement results on aircraft obtained by genetic algorithm

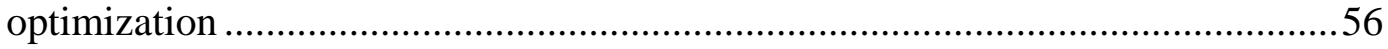

Table 6.2 Antenna placement results on aircraft obtained by particle swarm optimization

Table 6.3 Antenna placement results on ship obtained by genetic algorithm optimization

Table 6.4 Antenna placement results on ship obtained by particle swarm optimization . .76

Table 7.1 Results of the radiation pattern analyses of aircraft antennas .97

Table 7.2 Results of the radiation pattern analyses of ship antennas 106 


\section{LIST OF FIGURES}

\section{FIGURES}

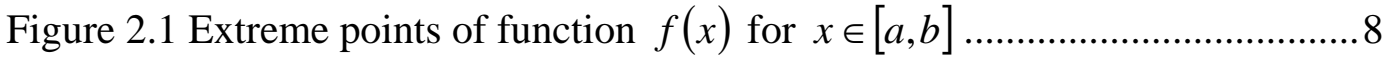

Figure 2.2 Flowchart of elitist genetic algorithm optimization ...........................13

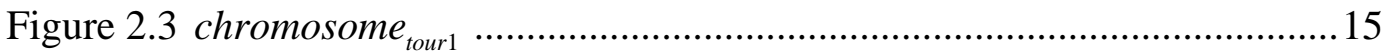

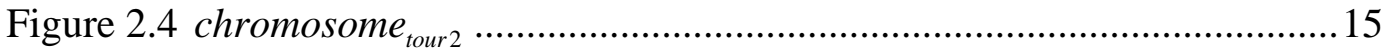

Figure 2.5 Phenotype-to-genotype encoding of $f(x, y)$.....................................17

Figure 2.6 Latin hypercube distribution (on the left) and uniform random

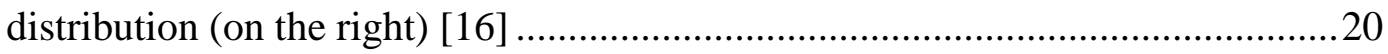

Figure 2.7 Tournament selection technique....................................................22

Figure 2.8 Single-point crossover technique .....................................................2 24

Figure 2.9 Two-point crossover technique ………..............................................24

Figure 2.10 Uniform random crossover technique .............................................25

Figure 2.11 Mutation operation in one-dimensional search space .......................26

Figure 2.12 Mutation by using flipping technique ............................................22

Figure 2.13 Mutation by using interchanging technique ...................................22

Figure 2.14 Mutation by using reversing technique ............................................28

Figure 2.15 Elitism, crossover and mutation in genetic algorithm [14] ................28

Figure 2.16 Flowchart of a basic particle swarm optimization .............................31

Figure 2.17 Position update mechanism of particle swarm optimization...............36

Figure 4.1 Dimensions of aircraft model .............................................................42

Figure 4.2 Dimensions of VHF antennas ..............................................................43

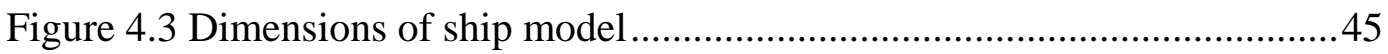

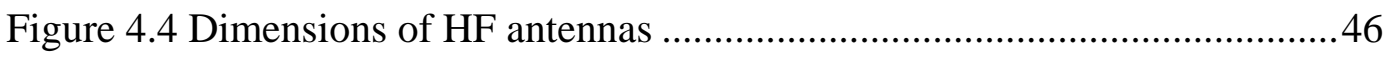


Figure 5.1 Two $\lambda / 4$ monopole antennas mounted on metallic circular cylinder of radius $10 \lambda[30]$

Figure 5.2 Coupling between monopole antennas vs. angular separation [30] .....49

Figure 5.3 The search space of VHF upper antenna...........................................50

Figure 5.4 The search space of VHF lower antenna............................................50

Figure 5.5 Coordinates of search spaces of HF fore and HF aft antennas..............51

Figure 6.1 Example showing how the simulation results are introduced as optimization parameters

Figure 6.2 Progress of the optimization run with an initial population generated by uniform random distribution technique and with a mutation rate of $60 \%$

Figure 6.3 Progress of the optimization run with an initial population generated by uniform random distribution technique and with a mutation rate of $30 \%$

Figure 6.4 Progress of the optimization run with an initial population generated by uniform random distribution technique and with a mutation rate of $1 \%$ .58

Figure 6.5 Progress of the optimization run with an initial population generated by Latin hypercube distribution technique and with a mutation rate of $60 \%$.......58 Figure 6.6 Progress of the optimization run with an initial population generated by Latin hypercube distribution technique and with a mutation rate of $30 \%$ \%......59 Figure 6.7 Progress of the optimization run with an initial population generated by Latin hypercube distribution technique and with a mutation rate of $1 \%$ .59

Figure 6.8 The placement yielding minimum final average coupling between VHF antennas (genetic algorithm optimization)

Figure 6.9 Progress of the optimization run with an initial swarm generated by uniform random distribution technique

Figure 6.10 Progress of the optimization run with an initial swarm generated by Latin hypercube distribution technique

Figure 6.11 The placement yielding minimum final average coupling between VHF antennas (particle swarm optimization)

Figure 6.12 Progress of the optimization run with an initial population generated by uniform random distribution technique and with a mutation rate of $60 \%$ 
Figure 6.13 Progress of the optimization run with an initial population generated by uniform random distribution technique and with a mutation rate of $30 \%$.......70 Figure 6.14 Progress of the optimization run with an initial population generated by uniform random distribution technique and with a mutation rate of $1 \%$.........70 Figure 6.15 Progress of the optimization run with an initial population generated by Latin hypercube distribution technique and with a mutation rate of $60 \%$.......71 Figure 6.16 Progress of the optimization run with an initial population generated by Latin hypercube distribution technique and with a mutation rate of $30 \%$.......71 Figure 6.17 Progress of the optimization run with an initial population generated by Latin hypercube distribution technique and with a mutation rate of $1 \%$.........72 Figure 6.18 The placement yielding minimum final average coupling between HF antennas (genetic algorithm optimization) .73

Figure 6.19 Progress of the optimization run with an initial swarm generated by uniform random distribution technique .77

Figure 6.20 Progress of the optimization run with an initial swarm generated by Latin hypercube distribution technique

Figure 6.21 The placement yielding minimum final average coupling between HF antennas (particle swarm optimization) .78

Figure 7.1 Far-field radiation patterns of monopole antenna at $30 \mathrm{MHz}$ .83

Figure 7.2 Far-field radiation pattern of VHF upper antenna at $30 \mathrm{MHz}$ .84

Figure 7.3 Far-field radiation pattern of VHF lower antenna at $30 \mathrm{MHz}$ .84

Figure 7.4 Far-field radiation patterns of monopole antenna at $40 \mathrm{MHz}$ .85

Figure 7.5 Far-field radiation pattern of VHF upper antenna at $40 \mathrm{MHz}$ .86

Figure 7.6 Far-field radiation pattern of VHF lower antenna at $40 \mathrm{MHz}$..............87

Figure 7.7 Far-field radiation patterns of monopole antenna at $50 \mathrm{MHz}$..............88

Figure 7.8 Far-field radiation pattern of VHF upper antenna at $50 \mathrm{MHz}$..............89

Figure 7.9 Far-field radiation pattern of VHF lower antenna at $50 \mathrm{MHz}$..............89

Figure 7.10 Far-field radiation patterns of monopole antenna at $60 \mathrm{MHz}$...........90

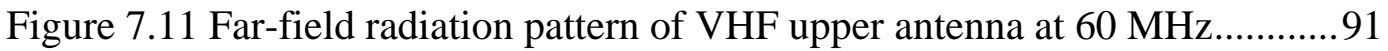

Figure 7.12 Far-field radiation pattern of VHF lower antenna at $60 \mathrm{MHz}$ .92 
Figure 7.13 Far-field radiation patterns of monopole antenna at $70 \mathrm{MHz}$...........93

Figure 7.14 Far-field radiation pattern of VHF upper antenna at $70 \mathrm{MHz}$............94

Figure 7.15 Far-field radiation pattern of VHF lower antenna at $70 \mathrm{MHz}$............94

Figure 7.16 Far-field radiation patterns of monopole antenna at $80 \mathrm{MHz}$...........95

Figure 7.17 Far-field radiation pattern of VHF upper antenna at $80 \mathrm{MHz}$............96

Figure 7.18 Far-field radiation pattern of VHF lower antenna at $80 \mathrm{MHz}$............97

Figure 7.19 Far-field radiation patterns of monopole antenna at $10 \mathrm{MHz}$..........100

Figure 7.20 Far-field radiation patterns of HF fore and aft antennas at $10 \mathrm{MHz} 101$

Figure 7.21 Far-field radiation patterns of monopole antenna at $20 \mathrm{MHz}$..........102

Figure 7.22 Far-field radiation patterns of HF fore and aft antennas at $20 \mathrm{MHz} 103$

Figure 7.23 Far-field radiation patterns of monopole antenna at $30 \mathrm{MHz}$.......... 104

Figure 7.24 Far-field radiation patterns of HF fore and aft antennas at $30 \mathrm{MHz} 105$ 


\section{CHAPTER 1}

\section{INTRODUCTION}

Communication requirements of today's modern aircraft and ship platforms are broad and diverse [1]. For that reason, they are equipped with various systems each of which takes a particular role in communication. These systems have similar RF characteristics and have many antennas operating at the same time in overlapping frequency bands. In such a case; there is a potential risk of platformbased system-to-system electromagnetic interference because of antenna-toantenna coupling. System-to-system interference may degrade the operational performance of the systems or damage the sensitive RF circuitry of these systems. One of the important steps followed in the design stages of aircraft and ship platforms before appealing filtering, blanking and etc., is determining an optimal antenna placement yielding minimum coupling between antennas that operate at the same time in overlapping frequency bands.

Antenna-to-antenna coupling can be calculated by various electromagnetic analysis tools easily. In spite of this simplicity, the geometrical structures of the platforms change and become more complex gradually, which make it difficult to determine an optimal antenna placement by trial and error or based on past experiences. For this reason, determining the optimal antenna placement can be considered as an "optimization problem" and this problem can be solved by global optimization techniques effectively. 
In electromagnetics, two important global optimization techniques, namely genetic algorithm optimization and particle swarm optimization are mostly used for the optimization of antenna, antenna array and RF component design, and optimization of radar cross section problems. Besides, there are studies in which genetic algorithm optimization is also used for the optimization of antenna placement. On the other hand, there is no considerable study in the literature in which the particle swarm optimization is used for the optimization of antenna placement problem.

In the study conducted by Kooper and Wood, genetic algorithm optimization is used for minimization of antenna-to-antenna coupling between VHF antennas on Boeing 747-200 aircraft [2]. Rather than a real aircraft model, a simple and smaller right circular cylinder is used for modeling the whole fuselage of the aircraft where antennas are mounted. By using Uniform Theory of Diffraction, coupling between the antennas and radiation pattern of the antennas are formulated analytically. The fitness function (function to be optimized) includes the coupling between the antennas and the scaled radiation patterns of the antennas as polar plots at certain frequencies. At the end, optimal antenna placements yielding minimum coupling and best two-dimensional radiation pattern performance are determined at distinct frequencies.

In another study performed by Aydemir, Günel and Üstüner optimization of coupling between two VHF-UHF antennas of F-4 Phantom aircraft by genetic algorithm optimization is aimed [3]. Aircraft is modeled based on its real physical properties. The fitness function includes coupling between the antennas calculated by Method of Moments method at three distinct frequencies. Different from the study of Kooper and Wood, radiation pattern performances of the antennas are not considered in the fitness function. Finally, optimal antenna placement yielding minimum coupling between the antennas at three distinct frequencies is determined. 
In the study of Suh, Lee, and Kim genetic algorithm is used for minimization of coupling between two ship board antennas [4]. Rather than a realistic ship model, a smaller and simpler ship model is used in the analyses. By using the combination of Method of Moments and Green's Function method, coupling between the antennas is calculated at a single frequency. One antenna is kept stationary and, position and angle of other antenna are changed during optimization process. At the end, optimal antenna placement yielding minimum coupling at a single frequency is obtained.

Moreover, genetic algorithm optimization is used by Barney, Knapil and Haupt in order to optimize the radiation pattern performance of a monopole antenna on a ship platform [5]. Rather than a realistic ship model, a very simple model constructed from basic geometric structures is used. In the analyses, minimum value of the directivity of the monopole antenna at a certain cross section and at a single frequency is tried to be maximized.

In this thesis work:

- Coupling between the antennas of two VHF radios on an aircraft platform and two HF radios on a ship platform is minimized by determining an optimal antenna placement, which yields minimum average coupling between the antennas over the whole frequency band of operation for each platform.

- Genetic Algorithm Optimization and Particle Swarm Optimization are used in determination of these optimal antenna placements. 
- Transient Solver of CST - MWS, which is based on Finite Integration Technique, is used in optimization of coupling between the antennas and in analyses of far-field ration patterns of the antennas.

- Aircraft and ship platforms are modeled based on the real physical dimensions and geometrical structures of F-4 Aircraft in Turkish Air Force Inventory and Patrol Boat in Turkish Naval Force Inventory respectively.

- For each platform, optimal antenna placements and coupling results determined by two different optimization techniques and performances of these optimization techniques are compared with each other.

- At the end of this thesis work; for each platform, far-field radiation pattern performances of the antennas at their optimal places are analyzed in terms of directivity and coverage.

Excluding introduction and conclusion parts, this thesis work consists of six chapters, each of which follows one another in a continual manner. In Chapter 2; first, "optimization” concept and terminology are introduced; then, features of local and global optimization techniques are given and compared with each other. Finally, detailed theory of two global optimization techniques used in this thesis work, namely "genetic algorithm optimization" and "particle swarm optimization" are explained. In Chapter 3, simulation tool, Computer Simulation Technology Microwave Studio (CST - MWS), used for the simulations of antenna placement and far-field radiation pattern analysis is briefly introduced. Chapter 4 details the steps followed in modeling of the platforms and their antennas in CST - MWS. In Chapter 5, the search spaces of antennas on the platforms are explained and illustrated in details. In Chapter 6, optimal antenna placements and coupling results determined by two different optimization techniques and performances of these optimization techniques are compared with each other for each platform. In 
Chapter 7, far-field radiation pattern performances of the antennas located at their optimal placements are analyzed in terms of directivity and coverage. Finally; in the last chapter, all conclusions obtained in this thesis work are summarized and further studies which can improve or extend the accomplishments of this thesis work are discussed. 


\title{
CHAPTER 2
}

\section{GLOBAL OPTIMIZATION TECHNIQUES: GENETIC ALGORITHM OPTIMIZATION AND PARTICLE SWARM OPTIMIZATION}

\begin{abstract}
Optimization simply means finding a "better" solution to a problem. It is the process of adjusting inputs of a phenomenon to obtain a desirable output. Most of the real-life problems are optimization problems, indeed. Scientists, engineers, manufacturers, financiers and even sociologists consult with different types of optimization techniques in order to obtain efficient solutions to their problems.
\end{abstract}

In mathematics, optimization deals with seeking for the minima or maxima of a function within a search space. In order to gain insight about the optimization concept in mathematics; first \& second derivatives test, which is a famous and a simple optimization technique used in mathematics, can be examined. In this test, the object is to determine the extreme points (minima and maxima) of a function over its search space. This simple optimization problem can be represented with an example; where the sample function, $f(x)$, to be optimized is depicted in Figure 2.1. In this example; the function $f(x)$ is assumed to be a one-variable, real valued, continuous and differentiable function, whose minima and maxima will be determined in the interval $x \in[a, b]$. Here, $f(x)$ is called "the objective or fitness or cost function”. It is important to note that, fitness is something to be maximized and cost is something to be minimized. In optimization terminology, 
both "to maximize the fitness" and "to minimize the cost" wordings refer to the same meaning which is "to optimize the objective function" [6].

In this example, $[a, b]$ is called "the search space". Solution to an optimization problem is searched over the search space. Boundaries of the search space are directly related with bounds on variables of the optimization problem. If there is no specification for the variables then the problem is called "unconstrained" optimization problem. For example, an unconstrained minimization problem can be defined as:

minimize $20+x^{2}-10 \cos (2 \pi x)$

On the other hand, if the variables have equality and/or inequality constraints on them, then the problem becomes a "constrained" optimization problem. For example; the previous unconstrained optimization problem can be transformed to a constrained one by introducing an inequality constraint on the variable such as:

minimize $20+x^{2}-10 \cos (2 \pi x)$, where $0 \leq x^{2}<1$

Since the minima and maxima of $f(x)$ will be determined in the interval $x \in[a, b]$, this problem is a constrained optimization problem.

During the first \& second derivatives test; initially, first derivative of the function is set to zero and extreme points satisfying this equation are extracted. Secondly, values of second derivative of $f(x)$ at those extreme points are checked whether they are greater or smaller than zero. If the value of second derivative is greater than zero at an extreme point, then that extreme point is a "minimum", and conversely if the value of second derivative is less than zero at an extreme point, then that extreme point is a "maximum". If the value of $f(x)$ at a maximum is 
greater than the values of $f(x)$ at other maxima, then this maximum is called "the global maximum" and each of the other maxima is called "the local maximum". Similar relationship also exists between "local minimum" and "global minimum". Namely, if the value of $f(x)$ at a minimum is less than the values of $f(x)$ at other minima, then this minimum is called "global minimum" and each of the other minima is called "the local minimum" (Figure 2.1).

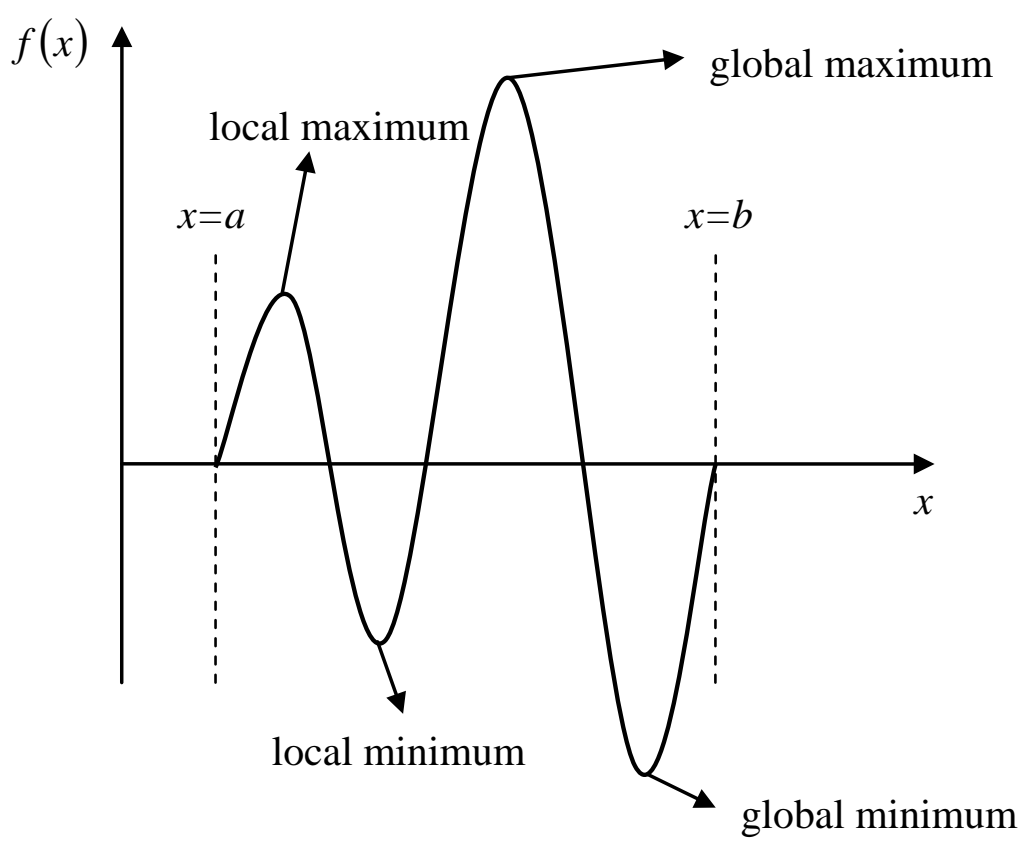

Figure 2.1 Extreme points of function $f(x)$ for $x \in[a, b]$

As in the example above, there may be both local and global solutions to an optimization problem. Similarly, optimization techniques can also be classified as local and global optimization techniques depending on their search strategies for extreme points. 
Local optimization techniques start searching an extreme point with an initial solution which is chosen randomly or systematically, and iteratively generate new solutions "within the neighborhood” of previous solutions. "A single” neighboring solution is generated at each iteration by "neighbor function" where the search strategy of the optimization is embedded in [7]. This optimization process continues until stopping criterion is reached.

Well-known local optimization techniques, which are also provided by CSTMWS, are the Quasi Newton, the Powell and the Nelder-Mead Simplex optimization techniques. The Quasi Newton optimizer requires approximated gradient information and the Powell optimizer requires partial derivative information to determine a search direction and to generate new solution at each iteration [8]. The Nelder-Mead Simplex optimizer does not need any gradient information to determine a search direction. Instead it uses a geometrical structure called "simplex" which adapts itself to the neighborhood, and focuses iteratively on to the extreme point [8], [9]. In addition to techniques specified above, there are many other local optimization techniques applied for the solution of optimization problems.

Local optimization techniques do not guarantee to find the global optimum. These techniques may be effective and computationally compact for smooth problems where global solution is not the prime concern [9]. However; these techniques generally are very sensitive to the choice of the initial point in the search space. If the initial point is not chosen close to the extreme point and the objective function is not plain, then even convergence to a local extreme point will not be easy.

On the other hand, global optimization techniques are preferable when the objective function is complex (not easily differentiable and/or having multiple extreme points) and pre-estimation of initial solution can not be easily made over a wide search space [8]. These techniques seek for the "best" solution to a 
problem among all possible good solutions. They generally follow populationbased search strategy. Therefore, they start searching by generating an initial population rather than a single solution as in the case of local optimization [10]. Then, they iteratively generate new populations by making use of the fitness values of the elements of previous populations. Elements of the populations are generated not only within the neighborhood of the previous solution, but also among whole search space. At each iteration, elements of the population move toward more optimal solutions and optimization stops when a stopping criterion is achieved like it happens in local optimization [11].

Global optimization techniques are classified into many classes according to their methods of operation. Throughout this thesis work, two of the well-known natureinspired (also called bio-inspired) global optimization techniques, "genetic algorithm optimization” and "particle swarm optimization”, were used in order to minimize antenna-to-antenna coupling on aircraft and ship. Both of the techniques are population-based optimization algorithms representing the processes in nature which are successful at optimizing natural phenomena [11]. These optimization techniques are explained in detail in Sections 2.1 and 2.2 respectively.

Besides local and global optimization, optimization techniques can be categorized into many branches like unconstrained and constrained optimization (Equations 2.1 and 2.2), single and multi variable optimization, single and multi objective optimization and etc. Nevertheless, none of these branches are mutually exclusive.

\subsection{Genetic Algorithm Optimization}

Nature's perfect balance and strong capability of optimization of natural phenomena have been source of inspiration for scientists in solving hard technical 
optimization problems. Thus, they developed many natural (nature-inspired) optimization techniques which imitate the optimization processes in nature.

One of the famous natural optimization techniques is the genetic algorithm optimization (which is also called "genetic algorithm" in short). The genetic algorithm is a member of the larger class of "evolutionary algorithms" and it searches for global optimum by using techniques derived from principles of genetics, natural selection and evolution [11]. The genetic algorithm was developed in 1975 by John Holland who is an American scientist and Professor of Psychology and Professor of Electrical Engineering and Computer Science at the University of Michigan, Ann Arbor. In his book "Adaptation in natural and artificial systems”, he described how to apply the principles of natural selection and evolution, theory of Charles Darwin, and the principles of genetics to real-life optimization problems [12]. Holland's idea has been further developed and popularized by one of his students, David Goldberg, who used genetic algorithm to solve a pipeline optimization problem in 1989 [13]. Nowadays; thanks to the computer technology, genetic algorithm stands as a powerful tool for solving global optimization problems. This algorithm can be used for the solution of optimization problems that are not suitable for standard optimization algorithms, including problems in which the objective function is discontinuous, nondifferentiable, stochastic, or highly nonlinear [14].

\subsubsection{Theory of Operation}

Individuals and operators used for searching the global optimum in genetic algorithm are analogous with individuals and operators in genetics [11]. Since the genetic algorithm is a population based global search algorithm, it starts searching the global optimum by generating an initial population which is composed of many individuals called "chromosomes". Each chromosome is considered as a candidate for the optimal solution. Then, the algorithm evaluates the "fitness" of 
each chromosome and "selects" the fitter ones by a special selection strategy to use them in creation of the next generation. A part of the selected chromosomes is directly transferred to the next generation without any change. These chromosomes are called "elite" chromosomes. The other part of the selected individuals is first used in "crossover" (mating) and then in "mutation" operations for creation of new individuals for the next generation. According to Holland; individuals of the newer generations, generated by utilizing specific rules of genetics like “elitism”, “crossover” and "mutation” on current population, are supposed to be fitter than their parents [12]. At the end, fitness of each individual in the new generation is evaluated and stopping criterion is checked. If stopping criterion is met algorithm stops. If not, then the algorithm continues to "select" individuals to use them as parents for the next generation. Flowchart of elitist genetic algorithm optimization is shown in Figure 2.2.

\subsubsection{Chromosomes}

Basic elements of the genetic algorithm are individuals called "chromosomes". Populations are composed of these basic elements. Each chromosome is a solution to the optimization problem. Main aim of the algorithm is to find a chromosome (solution) which optimizes the objective (fitness or cost) function. If the objective function has $n_{\mathrm{var}}$ variables (if optimization problem is $n_{\mathrm{var}}$ - dimensional) then the chromosome is defined as an array of $n_{\text {var }}$ variables. For example; for an optimization problem with an objective function $f$ having two variables of $x$ and $y$ ( $n_{\mathrm{var}}=2$ ), belonging to set of real numbers, the chromosome can be defined as a $1 \times 2$ row vector such as [11]:

chromosome $=[x, y]$ where $x, y \in \mathfrak{R}$ 


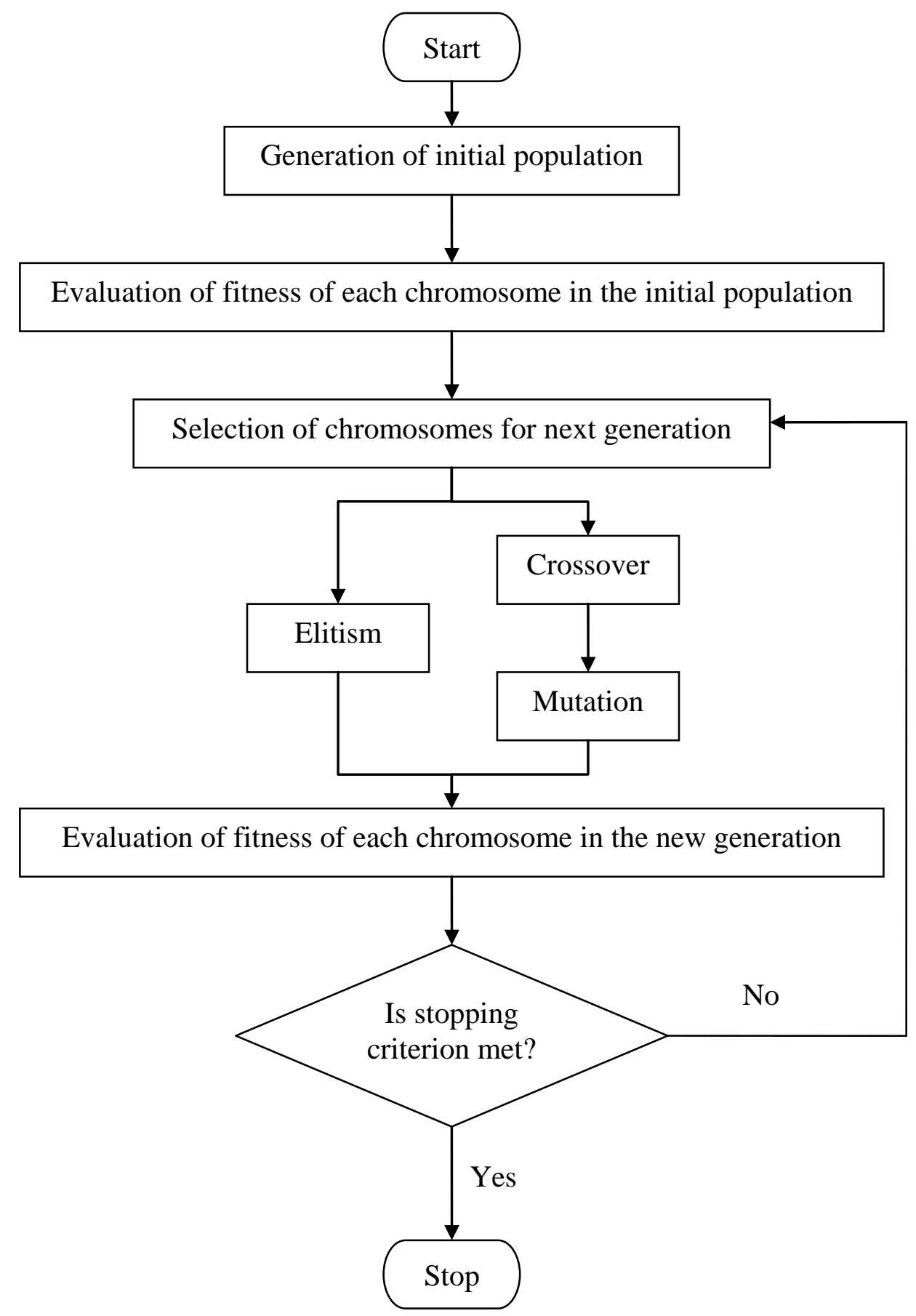

Figure 2.2 Flowchart of elitist genetic algorithm optimization 
Fitness value of the chromosome is determined by evaluating it in objective (fitness) function.

fitness of chromosome $=f($ chromosome $)=f(x, y)$

In most of the optimization problems and in the antenna placement problem solved by genetic algorithm throughout this thesis; variables of chromosomes, and hence the search space are defined as real numbers. However; according to type of optimization problem; these variables can also be characters, descriptive words and etc. For example, in order to solve Traveling Salesman Problem, which is a famous optimization problem in computer science, by genetic algorithm; variables are defined as an array of city names. In this problem, the aim is to find the shortest possible tour of $n$ cities, that salesman visits each city no more than once [6]. If this problem is adapted to Turkey for cities İstanbul, Ankara, İzmir and Adana $(n=4)$, then the optimization variables can be defined such as:

chromosome $_{\text {tour } 1}=[$ Ankara, İstanbul, İzmir, Adana $]$

chromosome $_{\text {tour } 2}=[$ Ankara, İzmir, İstanbul, Adana $]$

chromosome $_{\text {tour } 1}$ and chromosome $e_{\text {tour } 2}$ are depicted in Figure 2.3 and Figure 2.4. 


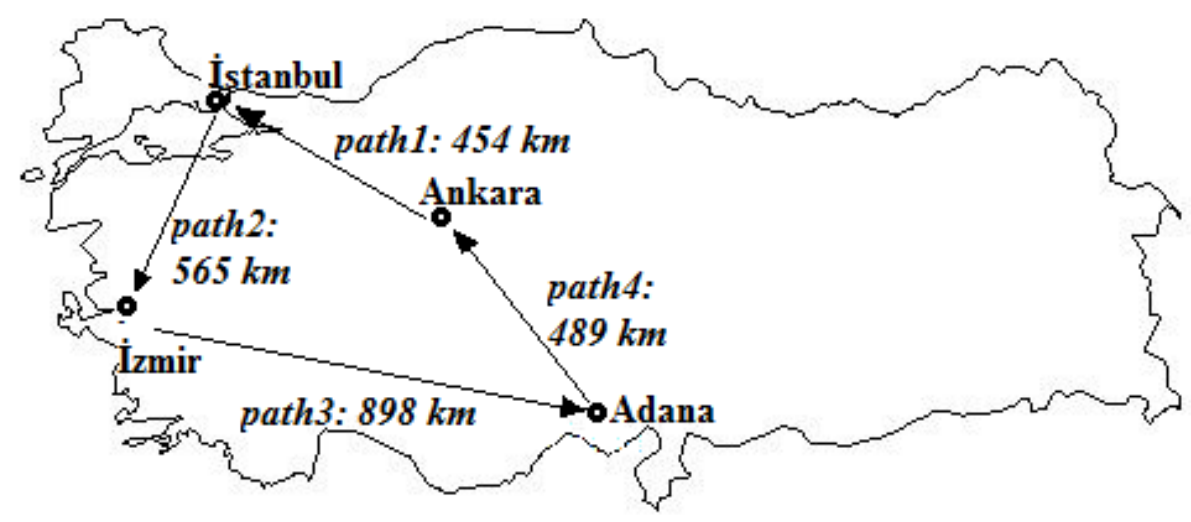

Figure 2.3 chromosome $_{\text {tour } 1}$

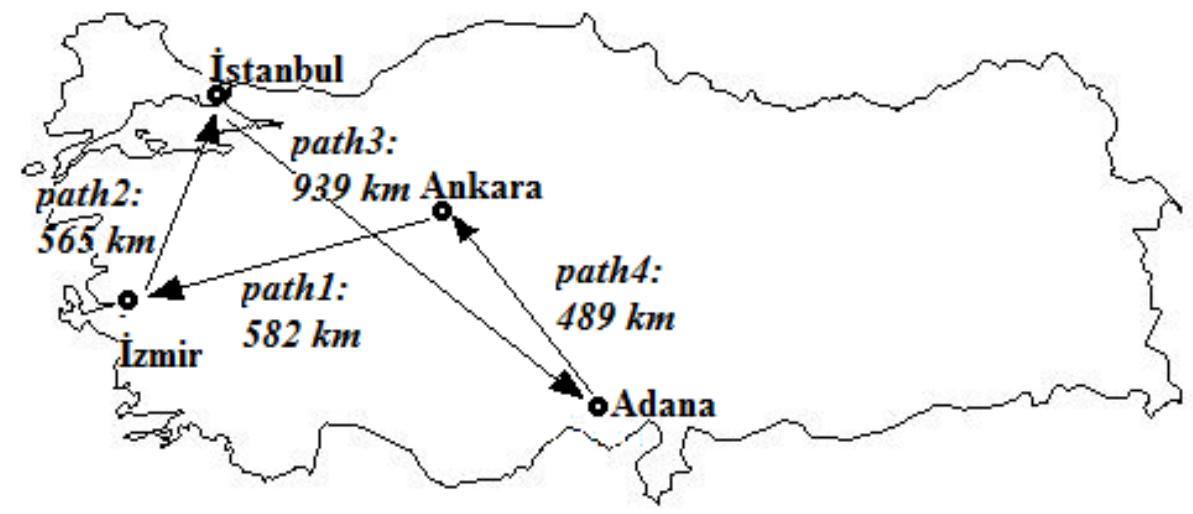

Figure 2.4 chromosome $_{\text {tour } 2}$

According to paths given in Figure 2.3 and Figure 2.4, fitness values of chromosome $_{\text {tour } 1}$ and chromosome tour $2_{2}$ are evaluated as:

$$
\begin{array}{rl}
{\text { fitness of } \text { chromosome }_{\text {tour } 1}} & =454 \mathrm{~km}+565 \mathrm{~km}+898 \mathrm{~km}+489 \mathrm{~km} \\
& =2406 \mathrm{~km} \\
{\text { fitness of } \text { chromosome }_{\text {tour } 2}} & 582 \mathrm{~km}+565 \mathrm{~km}+939 \mathrm{~km}+489 \mathrm{~km} \\
& =2575 \mathrm{~km}
\end{array}
$$


It is important to note that since this is a minimization problem, chromosome tour 1 is fitter than chromosome ${ }_{\text {tour } 2}$.

\subsubsection{Encoding of Chromosomes (Phenotype-to-Genotype Encoding)}

In genetic algorithm terminology; a chromosome is also called "phenotype" and the search space is also called "phenome" [15]. Selection operation is done based on fitness values of phenotypes. However; in order to do crossover and mutation operations on phenotypes, they should be encoded by binary arrays. The genetic representation of a phenotype formed after encoding is called "genotype". In binary encoding of a phenotype to a genotype, each variable of the phenotype is assigned to a unique bit string of a certain length called "gene". The bounds (constraints) of variables also apply to genes. The space in between lower and upper bounds of variables are divided into intervals that are represented genetically by gene's bit string. If the length of bit string of a gene is $n_{b i t s}$, then space in between lower and upper bounds of a variable can be divided into $2^{n_{\text {bis }}}-1$ intervals each of whose size is $\frac{\text { (upper bound }- \text { lower bound })}{2^{n_{\text {bis }}}-1}[6]$.

For example, for the constrained optimization problem given in Equation 2.9, space in between lower and upper bounds of each variable can be divided into 3 intervals if length of bit string of a gene, $n_{b i t s}$, is chosen as 2. Phenotype-togenotype encoding of the search space of this optimization problem for $n_{b i t s}=2$ is depicted in Figure 2.5.

minimize $f(x, y)$ where $x, y \in \mathfrak{R}$ and $a \leq x \leq b, c \leq y \leq d$ 


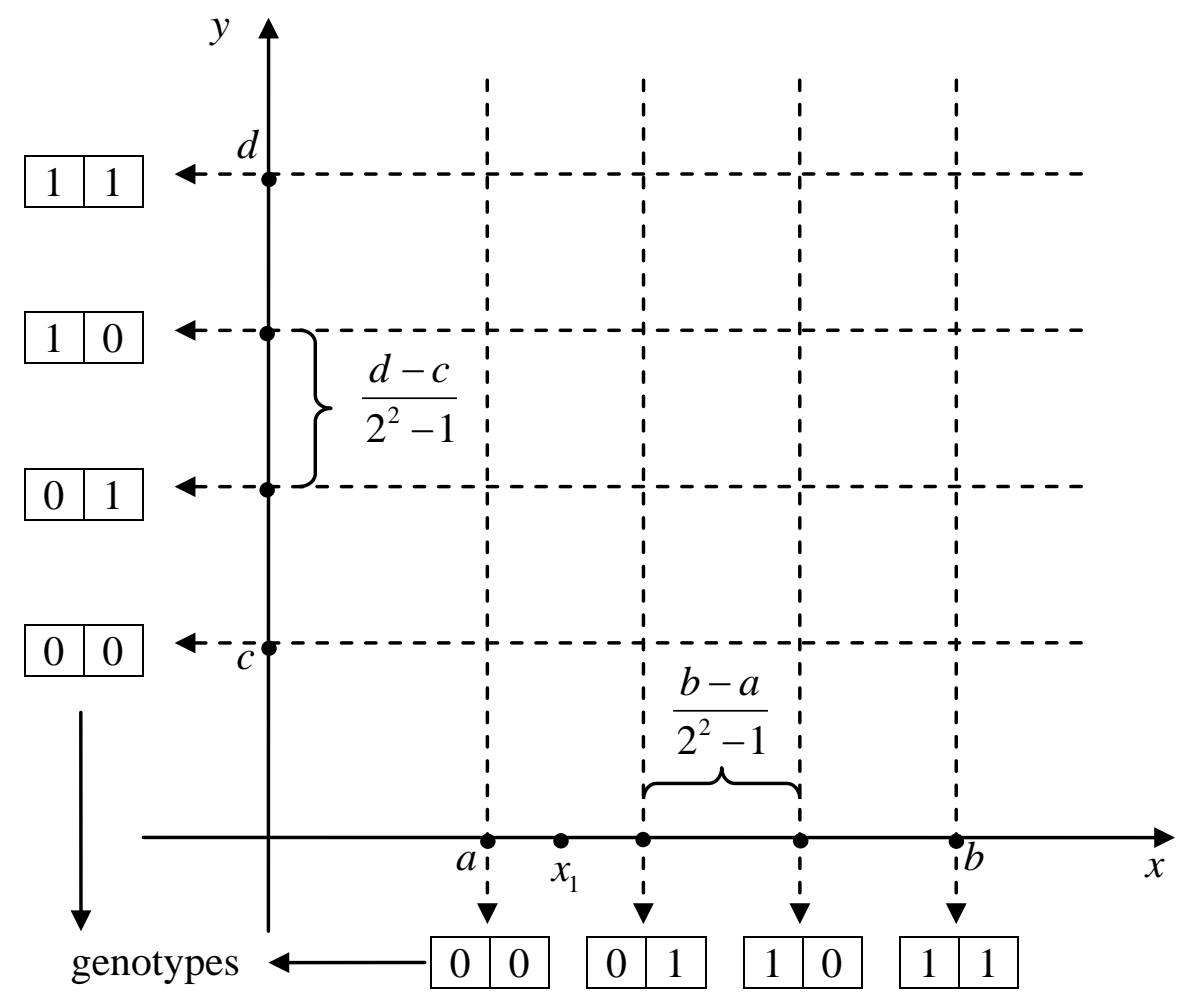

Figure 2.5 Phenotype-to-genotype encoding of $f(x, y)$

Genes which, in pair, form genotypes and corresponding variables which, in pair, form phenotypes are listed in Table 2.1.

For instance, according to this table:

- $\quad$ chromosome $(a, d)$ is genetically represented by chromosome $(\underbrace{00}_{\text {gene-x }}, \underbrace{11}_{\text {gene-y }})$,

- $\quad \operatorname{chromosome}(b, d)$ is genetically represented by chromosome $(\underbrace{11}_{\text {gene-x }}, \underbrace{11}_{\text {gene- } y})$ and etc. 
Table 2.1 Phenotype-to-genotype encoding of $f(x, y)$

\begin{tabular}{|l|c|l|c|}
\hline $\begin{array}{c}\text { variable- } x \\
\text { (phenotype) }\end{array}$ & $\begin{array}{c}\text { gene- } x \\
\text { (genotype) }\end{array}$ & $\begin{array}{c}\text { variable- } y \\
\text { (phenotype) }\end{array}$ & $\begin{array}{c}\text { gene- } y \\
\text { (genotype) }\end{array}$ \\
\hline$a$ & 00 & $c$ & 00 \\
\hline$a+\frac{b-a}{2^{2}-1}$ & 01 & $c+\frac{d-c}{2^{2}-1}$ & 01 \\
\hline$a+2 \times\left(\frac{b-a}{2^{2}-1}\right)$ & 10 & $c+2 \times\left(\frac{d-c}{2^{2}-1}\right)$ & 10 \\
\hline$a+3 \times\left(\frac{b-a}{2^{2}-1}\right)=b$ & 11 & $c+3 \times\left(\frac{d-c}{2^{2}-1}\right)=d$ & 11 \\
\hline
\end{tabular}

In this example, each continuous variable space (i.e. $a \leq x \leq b$ and $c \leq y \leq d$ ) is encoded by $2^{n_{\text {bis }}}=2^{2}=4$ discrete genes. Therefore, two-variable continuous search space is represented genetically by sixteen $\left(2^{n_{b i s}} \times 2^{n_{b i s}}=4 \times 4=16\right)$ discrete genotypes. Since the continuous search space is converted into an equivalent discrete space, there may occur "encoding error" (or discretization error) while encoding a phenotype to a genotype. For example, according to Figure 2.5, any $x$-value $\left(x_{1}\right)$ falling between $a$ and $a+\frac{b-a}{2^{2}-1}$, firstly is assigned to $a$ (the lowest value of that interval) or to $a+\frac{b-a}{2^{2}-1}$ (the highest value of that interval) and then encoded to its corresponding gene according to Table 2.1. Encoding error for $x_{1}$ is evaluated as $\left|x_{1}-a\right|$ or $\left|x_{1}-a+\frac{b-a}{2^{2}-1}\right|$. It is important to note that, if the length of bit string of a gene, $n_{b i t s}$, is increased, the space in between lower and upper bounds of variables will be represented by more genes. 
Moreover, increasing the number of bits of a gene would increase the number of intervals and reduce the size of intervals and hence "encoding error" [11].

\subsubsection{Initial Population}

Initial population is created so that all possible solutions in the search space are presented in. Except cases where it is possible to make a strong initial estimation for the location of optimal solution over the search space, initial population is created "randomly" [6]. Throughout this thesis work, two different random creation functions which are based on "uniform random distribution" and "Latin hypercube distribution” are used for the generation of initial population for genetic algorithm optimization problems. In Latin hypercube distribution technique; if the number of individuals in the population is $n_{\text {pop }}$ and the number of variables is $n_{\text {var }}$, then the search space is divided into $n_{\text {var }}$-dimensional, $\left(n_{p o p}\right)^{n_{\mathrm{var}}}$ identical sections. For instance; for two-dimensional search space case,

search space is divided into $\left(n_{\text {pop }}\right)^{2}$ identical sections which are in the form of squares. These square sections form rows and columns, and individuals are created such that there is only one individual in each row and each column. Similar procedure applies for search spaces having dimensions more than two. On the other hand, in uniform random distribution technique, there is no division of search space into intervals. In this distribution technique, the only criterion is that; each point in the search space has same probability to be chosen as a solution [8]. An example showing the differences between two distribution techniques are shown in Figure 2.6. 

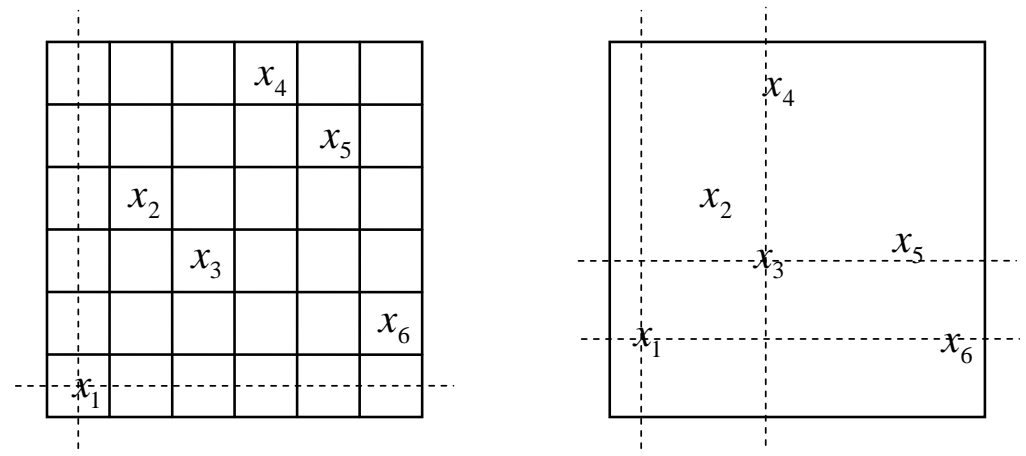

Figure 2.6 Latin hypercube distribution (on the left) and uniform random distribution (on the right) [16]

Size of the population also is an important parameter to sample the solution space effectively. If the size of the population increases, it becomes easier to explore whole search space and to converge to the optimal solution. However; time required to converge to the optimal point, memory used, cost of genetic algorithm also increase with the size of the population. Population size of around 100 individuals is generally used in genetic algorithms, but this size can be changed according to time, cost and memory limit of the algorithm [6].

\subsubsection{Selection, Crossover and Mutation}

As previously stated in the beginning of this section; after creation of initial population, the genetic algorithm uses three main types of operations (selection, crossover and mutation) at each iteration in order to create the next generation from the current population. Creation of initial population, selection of individuals, crossover and mutation operations all have "randomness" property where the power of genetic algorithm in finding global optimum comes from. 


\subsubsection{Selection}

Selection is a process of choosing a group of individuals from current population in order to use them as "parents" for the next generation. This operation is a random process which selects individuals based on their fitness values. According to Holland [12], who used "survival of the fittest" principle of Darwin as a baseline of genetic algorithm, parents which have higher fitness values will produce fitter children; and these children will evolve to better states through iterations. Therefore, Holland states that the next generation should be composed of the fitter parents and their children. Moreover, the parents who have lower fitness values should be discarded from the population. As a result; in selection operation, fitter individuals have higher chance to be selected as parents for the next generations.

According to selection strategy used throughout this thesis, half of the population which is composed of the fittest individuals is "directly transferred" to the next generation as parents. This operation is called "elitism" and used in order to prevent loss of fitter individuals (loss of healthy data) during crossover and mutation operations. The individuals in the other half, which will be involved in crossover and mutation operations, are selected by "tournament selection technique”. According to this technique, a small group of chromosomes is randomly selected from the current population and a tournament is made among that group. The chromosome with the highest fitness value wins the tournament at that group and selected as a parent. Tournament selections continue until desired number of parents is created [11]. Figure 2.7 depicts an example showing the tournament selection technique. According to Figure 2.7; at each tournament, three individuals are picked from the current population of eight individuals out and fitness values of these individuals are compared with each other. Among three individuals, the one with the highest fitness value is the winner of that tournament 
and is selected as a parent. Since the current population is composed of eight individuals, total number of eight tournaments is done in order to select eight parents.

The other well-known selection techniques are "roulette-wheel selection”, "rank selection" and "random selection". Roulette wheel selection technique selects individuals based upon their fitness values relative to the fitness of the other individuals in the population. Rank selection technique sorts the individuals according to their fitness values and selects them based on their rank in the population [6]. Random selection technique is very easy to implement since it just randomly selects the individuals from the current population.

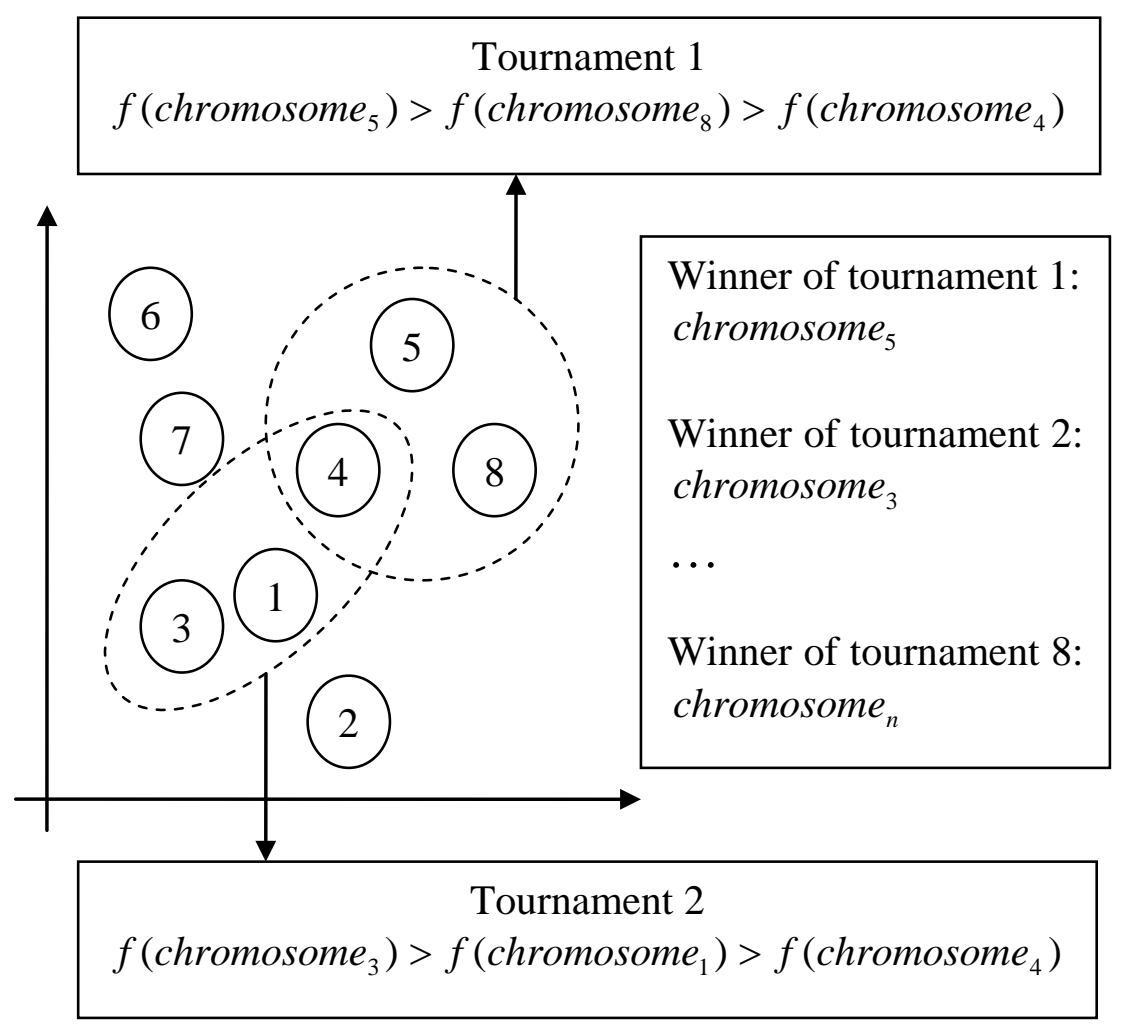

Figure 2.7 Tournament selection technique 


\subsubsection{Crossover}

After fitter individuals are selected; a part of them, excluding elite members, are mated to create children (new individuals) for the next generation. The fraction of selected individuals to which crossover will be applied is determined by "crossover rate" or "crossover probability". If crossover rate is too high, fitter parents may be lost during crossover operation. On the other hand, if crossover rate is too low, sufficient number of new offspring can not be produced so that convergence to the optimal solution may be slow [11]. Typically crossover probability between 0.6 and 0.8 is found to work best in most optimization problems [17]. In CST- MWS crossover rate is adopted according to the structure of individuals at each iteration.

Three well-known crossover techniques are "single-point crossover", "two-point crossover”, “uniform random crossover”. Generally, two individuals are mated in each of these crossover techniques. According to the single and two-point crossover techniques, a part of genetic information (binary code) of the first individual (genotype) is transferred to the second individual and in a like manner a part of genetic information of the second individual is transferred to the first individual. In single-point crossover technique, binary chromosomes are split into two arrays at the same crossover points and arrays after these points are exchanged. In two-point crossover technique, two crossover points are determined and the array between these points is exchanged between two parents. Examples showing the bit transfer mechanisms of these two techniques are depicted in Figure 2.8 and Figure 2.9 [6]. 


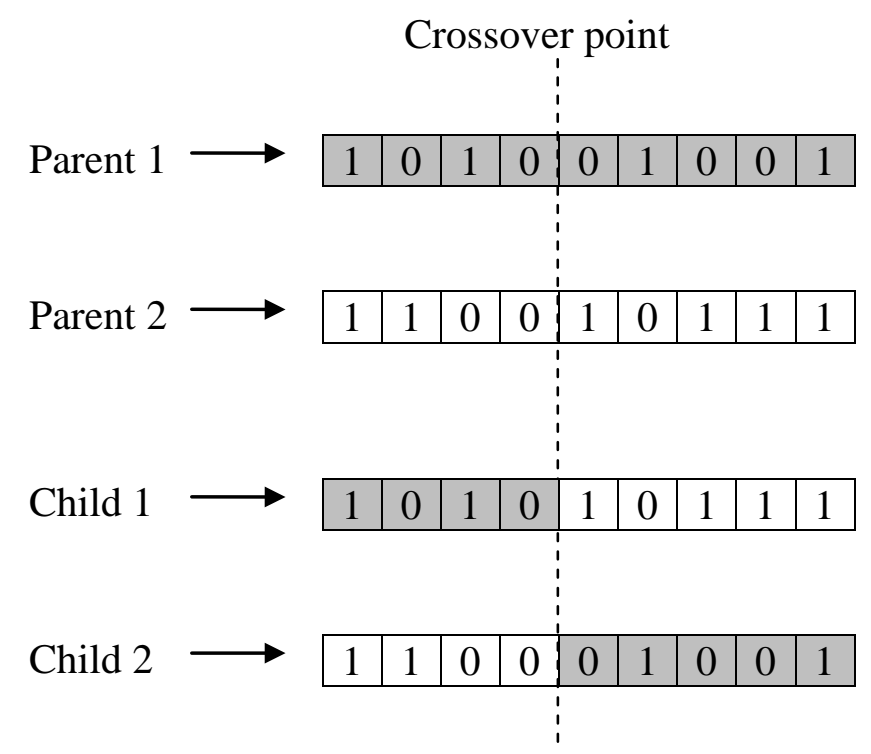

Figure 2.8 Single-point crossover technique

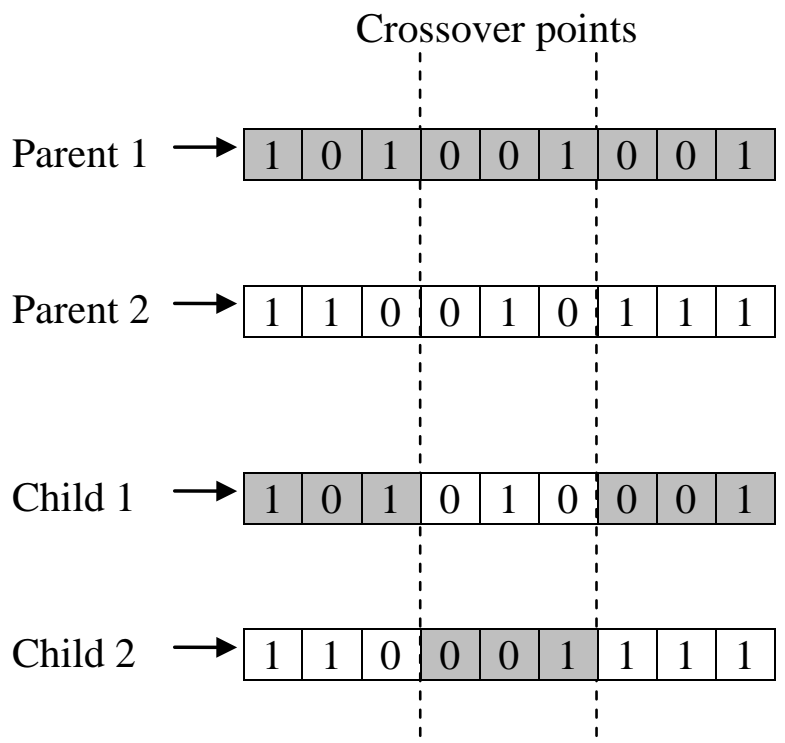

Figure 2.9 Two-point crossover technique 
Uniform random crossover technique, which is used throughout this thesis, creates offspring by interchanging the bits of the parents using a randomly generated binary "crossover mask" of the same length with the parents. This mask determines the bits which will be interchanged between parents and is created randomly such that the bits of the parents generally have a probability of 0.5 to be interchanged. In crossover mask, "ones” mean that the corresponding bits will be interchanged between parents and "zeros" mean that bits will not be interchanged. After interchanging operation is completed, two new children are generated from two parents. An example showing the interchange mechanism of this technique is depicted in Figure 2.10.

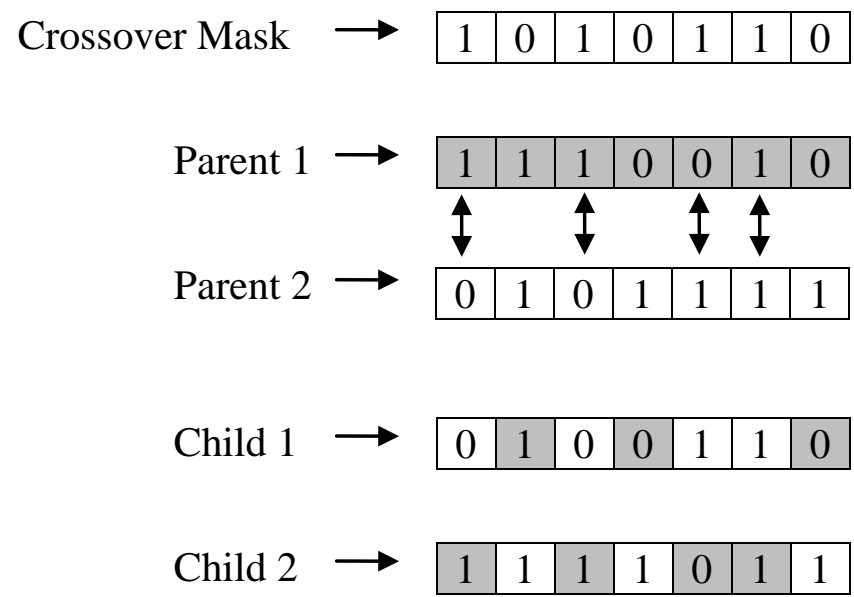

Figure 2.10 Uniform random crossover technique

\subsubsection{Mutation}

Mutation operation is as important as crossover operation for genetic algorithm. After crossover operation, there is a possibility that the individuals get stuck in a local optimum point. In order to prevent the algorithm from pre-mature 
converging to a local optimum before exploring the entire search space, mutation operation is done over a fraction of individuals in the population [6]. In mutation operation, a part of the binary chromosome is altered randomly so that a new chromosome with a totally different genetic structure is created. By this way, diversity in the population is maintained and new solutions that are not present in the genetic makeup of the population after crossover are explored. A graphical example showing how mutation works for one-dimensional problem space is given in Figure 2.11.

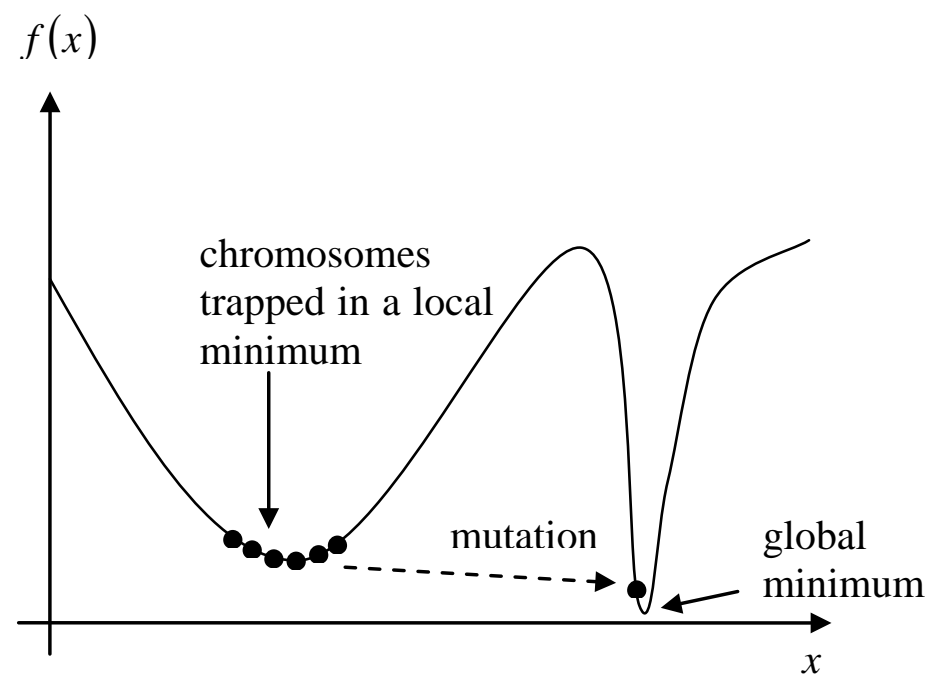

Figure 2.11 Mutation operation in one-dimensional search space

In CST - MWS, mutation is performed with a predefined mutation rate on the individuals who have very similar genetic characteristics. Mutation rate specifies the probability that a mutation occurs on one of the similar individuals.

Mutation can be performed in many ways by using such as "flipping", “interchanging” and "reversing” techniques [6]. All three techniques use similar 
mutation procedures to create a new child from a parent. In flipping technique, a "mutation mask" is generated and bits of the individual are changed according to that mask. In mutation mask, “ones” mean that the corresponding bits will be reversed and "zeros" mean that those bits will not be changed. In interchanging technique, two bits in the binary individual are chosen randomly and interchanged. In this technique, if the bits chosen are same then the individual remains unchanged. Finally, in reversing technique; a reversing point is chosen and the bits next to that point are reversed. Examples showing these three mutation techniques are depicted in Figure 2.12, Figure 2.13 and Figure 2.14.

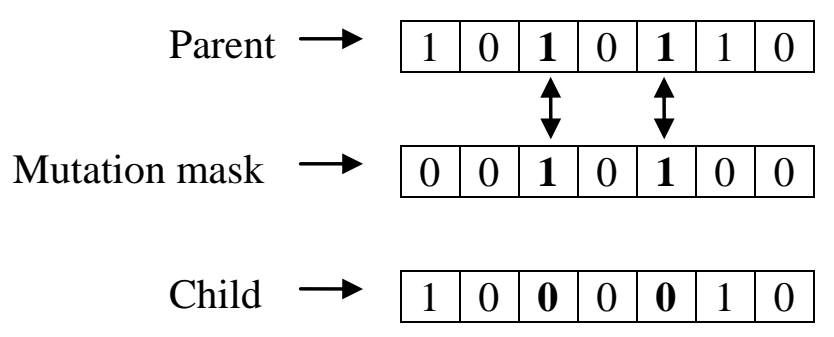

Figure 2.12 Mutation by using flipping technique

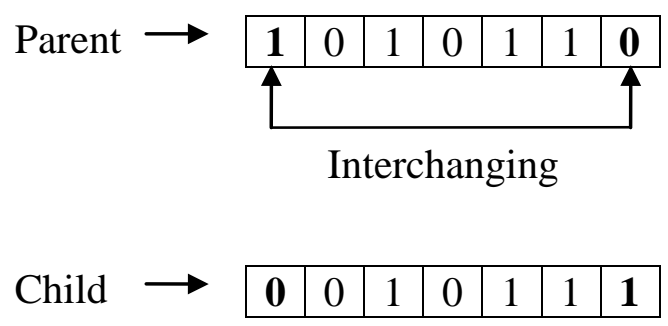

Figure 2.13 Mutation by using interchanging technique 


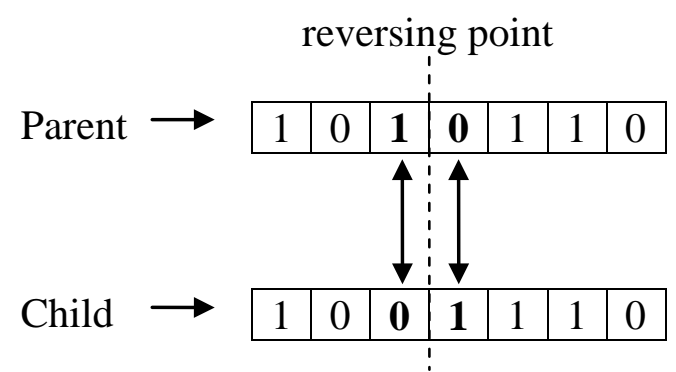

Figure 2.14 Mutation by using reversing technique

Elitism, crossover and mutation operations are illustrated in Figure 2.15.

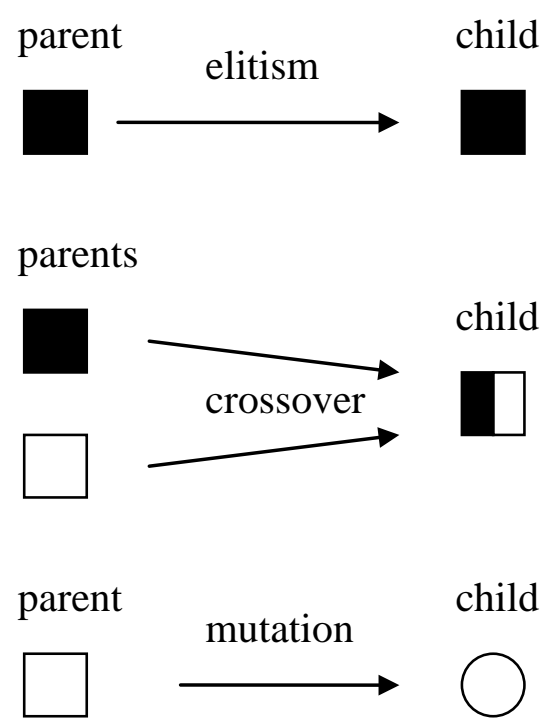

Figure 2.15 Elitism, crossover and mutation in genetic algorithm [14] 


\subsubsection{Stopping Criterion}

At each iteration; after a new generation is created, stopping criterion of the algorithm is checked. If stopping criterion is met, algorithm stops. If not, then the algorithm continues to "select" individuals for the creation of the next generation. In CST - MWS, the algorithm stops when the fitness value of the best individual in the population reaches the limit specified as a target in the beginning or maximum number of iterations is reached [8].

\subsection{Particle Swarm Optimization}

The other famous global optimization technique used throughout this thesis work is the particle swarm optimization which is inspired by social behavior of "swarms" in the nature. The swarms like bird flock and fish school are very successful in finding food in nature [18]. They are in continuous interaction with each other while searching for food in a large area. Each bird or fish tells the location and quantity of the food that it found to the others. Thus, by taking into account the location and quantity data from each member of the swarm, whole swarm tends towards the optimum location where maximum amount of food is present. Particle swarm optimization technique searches for the global optimum by using the techniques derived from the social interaction in the swarms while searching for food.

The particle swarm optimization technique was first introduced in 1995 by James Kennedy, who is a social psychologist; and by Russell Eberhart, who is an electrical engineer. In their articles "Particle Swarm Optimization” and "A new optimizer using particle swarm theory”, they described the stages of development of their technique from a social study of swarms to an optimizer [18], [19]. They also discussed the basic concepts of this technique and the results obtained from 
applications upon which their technique performed successfully. Particle swarm optimization technique has been further developed by many researchers and has undergone many changes since its introduction in 1995. According to Kennedy and Eberhart, particle swarm optimization can be used in solution of nonlinear global optimization problems effectively. Moreover, it has a very simple concept and can be implemented by using primitive mathematical operators which make the technique computationally inexpensive in terms of both memory and speed requirements [18], [19].

\subsubsection{Theory of Operation}

Particle swarm optimization, like genetic algorithm, is a population based global search technique. It starts searching the global optimum by generating an initial swarm which is composed of many individuals called "particles" and converge to the global optimum iteratively. Each particle in the swarm is considered as a potential solution for the optimization problem. In contrast to what happens in genetic algorithm, the particles are not encoded by binary arrays. Instead, they are represented by "position" and "velocity" vectors. Particles iteratively change their positions (fly) among the search space and seek for the optimum solution. At each iteration, position of the particle is updated according to its velocity vector. Besides; at each iteration, the velocity vector of a particle is updated according to "previous best position of that particle" and "previous best position of the swarm”. By this way, particles are in continuous interaction with each other and determine their movements according to their best and swarm's best performance. At the end of each iteration, fitness values of particles are evaluated and stopping criterion is checked. If stopping criterion is met optimization process stops. If not; first, the velocity vectors of the particles and then, the position vectors of them are updated. Movement of the particles among the search space is continued until stopping criterion is met. Flowchart of a basic particle swarm optimization is shown in Figure 2.16. 


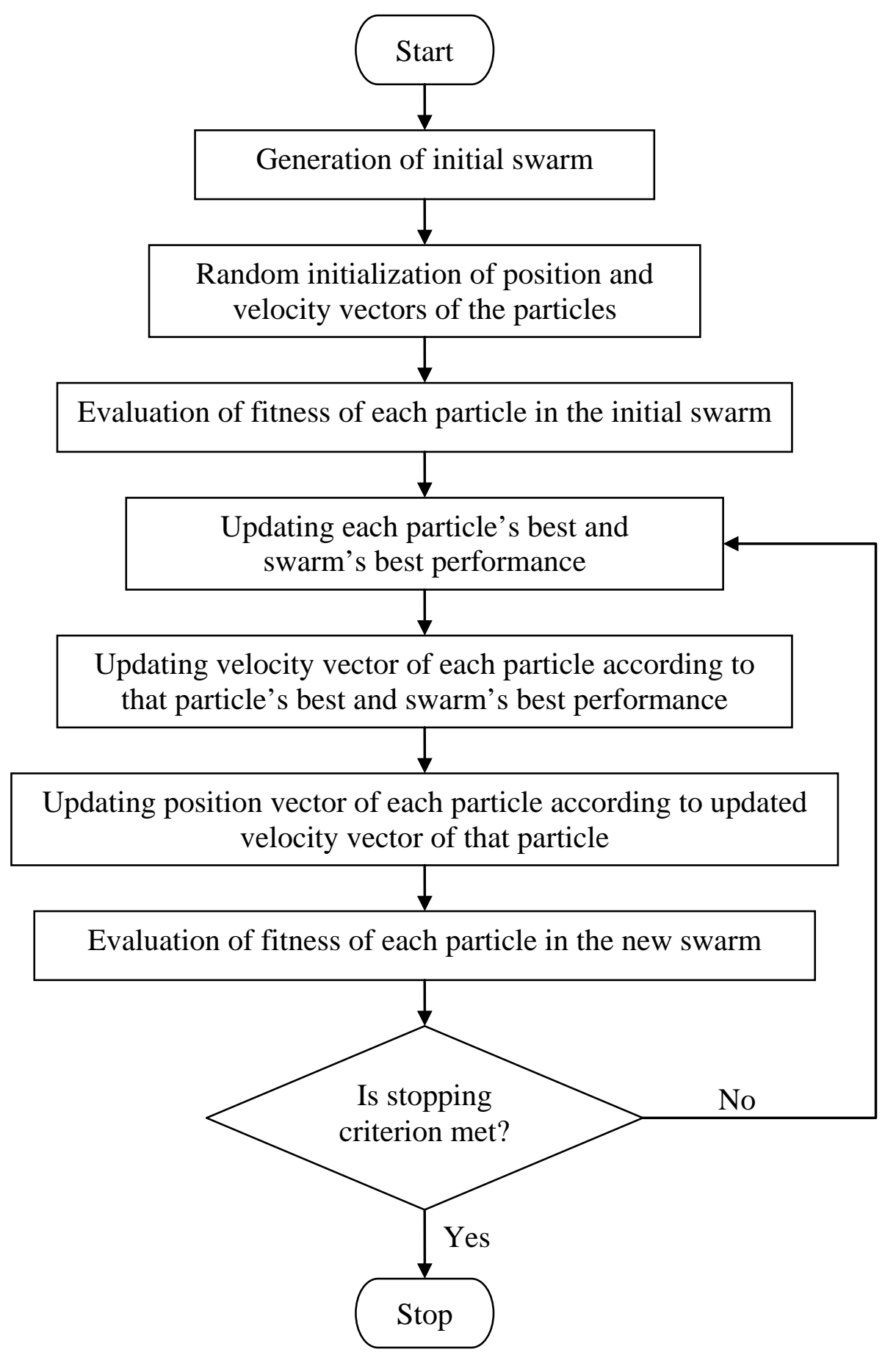

Figure 2.16 Flowchart of a basic particle swarm optimization 


\subsubsection{Particles, Velocity \& Position Vectors}

Basic elements of the particle swarm optimization are individuals called "particles”. Particles are potential solutions to the optimization problem and change their positions on the search space iteration by iteration in order to find a solution which optimizes the objective function. Unlike the genetic algorithm, particle swarm optimization has no genetic operators like crossover and mutation. Therefore, the particles do not need to be encoded into a special format.

Instead, each particle is represented by a "position" and a "velocity" vector at any iteration. These vectors are defined with respect to origin of the search space. If the objective function has $n_{\mathrm{var}}$ variables $\left(x_{1}, x_{2}, \ldots, x_{n_{\mathrm{var}}}\right)$ and the swarm has $n_{p o p}$

particles; then, the position and velocity vectors of $i^{\text {th }}$ particle at the $k^{\text {th }}$ iteration can be defined as:

position vector: $\vec{x}_{i}^{k}=\hat{a}_{x_{1}} x_{i, 1}^{k}+\hat{a}_{x_{2}} x_{i, 2}^{k}+\ldots+\hat{a}_{x_{n_{\text {var }}}} x_{i, n_{\text {var }}}^{k}$

velocity vector: $\vec{v}_{i}^{k}=\hat{a}_{x_{1}} v_{i, 1}^{k}+\hat{a}_{x_{2}} v_{i, 2}^{k}+\ldots+\hat{a}_{x_{n_{\text {var }}}} v_{i, n_{\text {var }}}^{k}$

where,

$k$ stands for the iteration number,

$i$ stands for the rank of the particle in the swarm and $i=1,2,3, \ldots, n_{\text {pop }}$

$\hat{a}_{x_{1}}, \hat{a}_{x_{2}}, \ldots, \hat{a}_{x_{n_{\text {var }}}}$ are unit vectors in the direction of variable axes. 
Fitness of a particle at the $k^{\text {th }}$ iteration is determined by evaluating its value in the objective function at its current position.

Fitness value of $i^{\text {th }}$ particle $=f\left(\vec{x}_{i}^{k}\right)=f\left(x_{i, 1}, x_{i, 2}, \ldots, x_{i, n_{\mathrm{var}}}\right)$

In most of the optimization problems and in the antenna placement problem solved by particle swarm optimization throughout this thesis; variables of particles, and hence the search space are defined as real numbers. However; according to the type optimization problem; these variables can also be characters, descriptive words and etc. In previous section, there is an example in where genetic algorithm is used for the solution of Traveling Salesman Problem. In this example, variables are city names therefore; chromosomes are defined as arrays of city names. Similarly, this problem can be solved by particle swarm optimization by defining the particles as arrays of city names.

\subsubsection{Initial Swarm}

In particle swarm optimization, initial swarm is created so that whole search space is sampled as uniformly as possible. Unless a strong initial estimation for the location of optimal solution over the search space can be made, position and velocity vectors of initial swarm are created "randomly". Throughout this thesis work, two different random creation functions are used for the generation of initial swarm for particle swarm optimization problems. These functions are based on "uniform random distribution” and "Latin hypercube distribution” techniques, which are explained in details in the previous section.

Size of the swarm also is an important parameter for sampling the solution space effectively. It is determined according to the dimension and difficulty of the 
problem. Swarm size in the range 20 to 50 particles are generally used in particle swarm optimization problems [20]

\subsubsection{Updating velocity \& position vectors}

In particle swarm optimization, each particle changes its position on the search space according to:

- its current position (inertial part)

- its previous best performance (cognitive part)

- previous best performance of the whole swarm (social part) [21], [22], [23].

At each iteration, velocity and position vectors of a particle are updated according to Equation 2.13 and Equation 2.14 respectively.

$\vec{v}_{i}^{k+1}=\underbrace{\omega \vec{v}_{i}^{k}}_{\begin{array}{c}\text { inertial } \\ \text { part }\end{array}}+\underbrace{c_{1} \operatorname{rand}_{1}{ }^{k}\left(\vec{p}_{i, \text { best }}^{k}-\vec{x}_{i}^{k}\right)}_{\begin{array}{c}\text { cognitive } \\ \text { part }\end{array}}+\underbrace{c_{2} \text { rand }_{2}{ }^{k}\left(\vec{g}_{\text {best }}^{k}-\vec{x}_{i}^{k}\right)}_{\begin{array}{c}\text { social } \\ \text { part }\end{array}}$

$\vec{x}_{i}^{k+1}=\vec{x}_{i}^{k}+\vec{v}_{i}^{k+1}$

Right side of Equation 2.13 consists of three parts. First part, which is called the “inertial part” [20], is the contribution of $i^{\text {th }}$ particle's previous velocity $\left(\vec{v}_{i}^{k}\right)$ to its next velocity $\left(\vec{v}_{i}^{k+1}\right)$. In this part, $\omega$ is the inertia weight which helps the particle to keep its inertia while updating its velocity vector. $\omega$ can be a positive constant number. In original particle swarm optimization which was introduced firstly in 1995, $\omega$ was set to be 1 [18]. Also, according to Shi and Eberhart [22], positive value of $\omega$ can change linearly in time. 
The second part; which is called the "cognitive part", is the contribution of $i^{\text {th }}$ particle's previous best experience and the third part; which is called the "social part", is the contribution of swarm's best experience in updating the current velocity vector of $i^{\text {th }}$ particle. According to Shi and Eberhart [22], the second part represents the private thinking of the particle itself and the third part represents the social interaction and collaboration among the particles.

The previous best position of the $i^{\text {th }}$ particle (up to $k^{\text {th }}$ iteration) is recorded and represented as vector $\vec{p}_{i, b e s t}^{k}$. The position of the particle in the whole swarm which has the best fitness value (up to $k^{\text {th }}$ iteration) is recorded and represented as vector $\vec{g}_{\text {best }}^{k} \cdot c_{1}$ and $c_{2}$ are positive constant numbers which are called "self confidence" and "swarm confidence" respectively [23]. $\operatorname{rand}_{1}{ }^{k}$ and $\operatorname{rand}_{2}{ }^{k}$ are random numbers uniformly distributed in the interval [0, 1]. These random numbers add stochasticity, which is an important property of particle swarm optimization algorithm in finding global optimum, to the vectors.

In Equation 2.13, $\left(\vec{p}_{i, \text { best }}^{k}-\vec{x}_{i}^{k}\right)$ is the difference between particle’s previous best position vector and particle's current position vector. Similarly, $\left(\vec{g}_{\text {best }}^{k}-\vec{x}_{i}^{k}\right)$ is the difference between swarm's previous best position vector and particle's current position vector. These difference vectors are weighted by $c_{1}$ rand $_{1}{ }^{k}$ and $c_{2}$ rand $_{2}{ }^{k}$ respectively and added to the current velocity vector of the particle in order to update it. At the end, updated velocity vector in Equation 2.13, is added to current position vector and the current position vector is updated by this manner (Equation 2.14). This process is repeated for each particle at each iteration until the stopping criterion is met. An example, showing position update mechanism of particle swarm optimization algorithm (Equation 2.13 and 2.14) for twodimensional ( $x$ and $y$ variables) search space, is illustrated in Figure 2.17. 


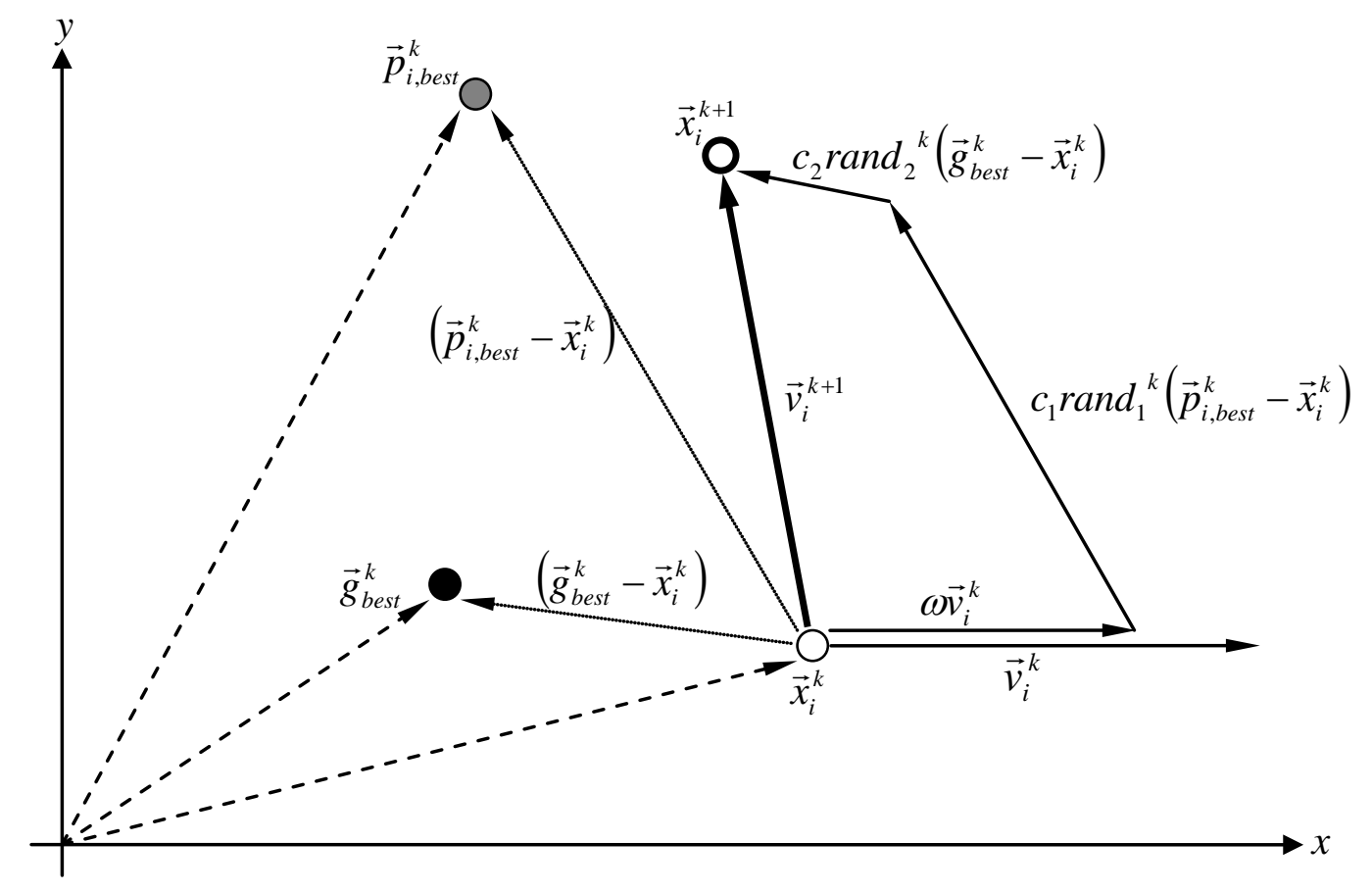

Figure 2.17 Position update mechanism of particle swarm optimization

In Equation 2.13, without the first part (while $\omega=0$ ) the velocity vector of a particle will be updated only by particle's previous best position and swarm's best position. In this case, the particle having the current best fitness value will not move since $\left(\vec{p}_{i, \text { best }}^{k}-\vec{x}_{i}^{k}\right)$ and $\left(\vec{g}_{i, \text { best }}^{k}-\vec{x}_{i}^{k}\right)$ will be zero. With no inertial component, other particles move fast toward this current best position unless a better position is found on the way. Therefore, search space shrinks fast through the iterations and algorithm resembles a local search algorithm in which there is a possibility of premature convergence to a local optimum [22]. On the other hand, it is obvious that, without the second and the third parts and with a constant $\omega$, the velocity vector of a particle will not be updated and the particle will move on a straight line with its initial speed until it hits the boundary of the search space. Due to the type of the problem inertia weight, $\omega$ : 
- may be a constant number

- may decrease from a high value to a low value in time

- may be chosen randomly from an interval in time

- may be adopted by an external controller in time [20], [23].

$c_{1}$ and $c_{2}$ are the parameters that determine the magnitude of random forces in the direction of particle's and swarm's previous best position vectors [20]. Magnitudes of $c_{1}$ and $c_{2}$ substantially affect the magnitude and direction of the velocity vector. They are usually chosen equal to each other and range from 0 to 4 [23]. In earlier particle swarm optimization researches, $c_{1}$ and $c_{2}$ were chosen to be 2 but in recent works, a usual choice for $c_{1}$ and $c_{2}$ is 1.494 [20], [23].

The magnitude of the velocity vector, $\left|\vec{v}_{i}^{k}\right|$, is bounded by $v_{\max }$ in order to damp the movement of the particle over the search space. Just as inertia weight, $v_{\max }$ may be a constant number or may differ due to the type of the problem. If $v_{\max }$ is chosen too large, movements of particles at each iteration will be too large and particles will search for the solution far away from the global optima. Moreover, if $v_{\max }$ is chosen too small, then there is a risk of the algorithm getting stuck in a local optima. The same as above also happens when $c_{1}$ and $c_{2}$ are chosen too large or too small [21].

Due to the optimization parameters used or due to the location of the global minimum, there is a possibility of some of the particles moving outside the search space, when their position vectors are updated. For example, if the location of the global optimum is near to the boundary of the search space and/or $v_{\max }$ is chosen too large, this problem may arise. In order to prevent particles to search for the global optimum outside the search space, various "boundary conditions" are used. The boundary conditions currently used in literature are absorbing, reflecting, 
invisible and damping boundary conditions [24]. These boundary conditions have different features to redirect the particles outside into the search space of interest and their performances are highly dependant on the type of the problem, size of the search space and location of the global optimum.

It is important to note that if $\vec{g}_{\text {best }}^{k}$ is defined not for the whole swarm but for the particles in the neighborhood, then the algorithm is called the "partial" particle swarm optimization algorithm.

In CST - MWS, $v_{\max }$ is chosen as a constant number according to the size of the search space. The other terms like $\omega, c_{1}$ and $c_{2}$ are chosen quasi-randomly from predefined ranges during the optimization process.

\subsubsection{Stopping Criterion}

At each iteration; after a new swarm is created, stopping criterion of the algorithm is checked. If stopping criterion is met algorithm stops. If not, algorithm continues updating each particle's velocity and position vectors. In CST - MWS the algorithm stops when the fitness value of the best individual in the population reaches the limit specified as a target in the beginning or maximum number of iterations is achieved [8]. 


\section{CHAPTER 3}

\section{SIMULATION TOOL}

Throughout this thesis, Computer Simulation Technology - Microwave Studio (CST - MWS), which is a simulation module of CST Studio Suite, is used for the simulations of antenna placement and far-field radiation pattern analysis. CST Studio Suite is the main simulation software product of CST Company, which was founded in 1992 in Darmstadt, Germany, and which develops and markets software tools for the simulation of electromagnetic problems in all frequency bands [25].

CST - MWS is a powerful three-dimensional electromagnetic simulation tool for the fast and accurate solutions of high frequency problems. It provides a solid modeling tool in where the components can be modeled with their real electrical and physical characteristics. It has ability to solve different types of electromagnetic problems by variety of different solvers operating in time and frequency domains. These solvers are Transient Solver, Frequency Domain Solver, Integral Equation Solver, Eigenmode Solver [26].

In this thesis study Transient Solver of CST - MWS, which is adequate for antenna placement and far-field radiation pattern analysis, is used. Main advantage of Transient Solver is that the entire broadband frequency behavior of the simulated problem can be obtained from only one calculation run. By this way broadband coupling between the antennas of aircraft and ship platforms were obtained from one single calculation run during optimization processes. 
Transient Solver of CST - MWS is based on Finite Integration Technique developed by Weiland in 1976/1977 [27]. This numerical method provides a universal spatial discretization scheme for Maxwell's equations in their "integral form” [28].

In order to solve these equations for the unknown electric and magnetic parameters numerically, a finite calculation domain (with boundary conditions) enclosing the problem space is defined and divided into orthogonal hexahedral grid cells. After that, Maxwell's equations in their integral form are discretized spatially for each of the hexahedral cells. For the whole problem space, discretized Maxwell's equations are arranged together and written in matrix form. Finally, this matrix is solved for the unknown electric and magnetic parameters according to boundary conditions. In the light of these solved parameters coupling between two antennas and far-field radiation pattern of an antenna can be calculated [28]. 


\title{
CHAPTER 4
}

\section{MODELING OF AIRCRAFT, SHIP AND THEIR ANTENNAS}

\begin{abstract}
Aircraft \& ship platforms and antennas placed on them are modeled in CST which provides a three-dimensional solid modeling tool in where structures can be modeled based on their real electrical and physical properties. This chapter details, firstly the modeling of aircraft platform and its VHF antennas and secondly the modeling of ship platform and its HF antennas.
\end{abstract}

\subsection{Modeling of Aircraft Platform and Its Antennas}

Aircraft platform and VHF antennas placed on it are modeled as follows:

- Both aircraft platform and its antennas are modeled in a problem space defined as "normal” which has:

o No electrical conductivity, $\sigma=0$ Siemens / meter

o Permittivity of free space, $\varepsilon_{0} \cong 8.85 \times 10^{-12}$ Farads / meter

o Permeability of free space, $\mu_{0}=4 \pi \times 10^{-7}$ Henrys / meter

- Problem space in which the aircraft and its antennas are modeled, is divided into about 300,000 hexahedral grid cells having smallest and largest side length of about $1.25 \mathrm{~cm}$ and $30 \mathrm{~cm}$ respectively. 
- Aircraft is modeled by primitive geometric structures in order to reduce simulation complexity and simulation time, based on the real physical dimensions and geometrical structure of F-4 Aircraft in Turkish Air Force Inventory.

- Aircraft has an overall length of $19.5 \mathrm{~m}$, wingspan of $12 \mathrm{~m}$ and fuselage width of $3 \mathrm{~m}$ (Figure 4.1).

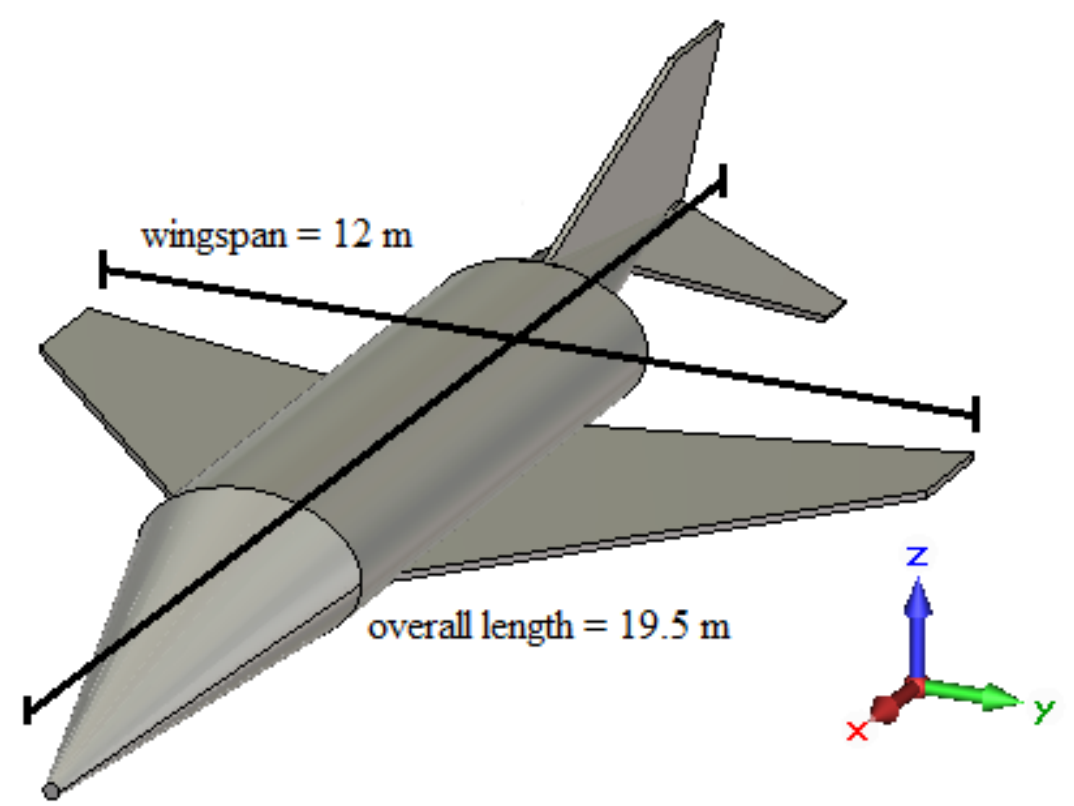

Figure 4.1 Dimensions of aircraft model

- VHF antennas are modeled as $\lambda / 4$ monopole antennas operating in $30-$ $88 \mathrm{MHz}$ frequency band. They have length of $1 \mathrm{~m}$ and radius of $0.625 \mathrm{~cm}$ (Figure 4.2). 
- VHF antennas are connected to aircraft and excited by discrete ports having length of $1.25 \mathrm{~cm}$ and impedance of $50 \Omega$. Discrete ports are considered to be lumped circuit elements with an internal resistor and a current source in parallel. These ports consist of a lumped element in the middle and two perfectly electric conducting wires connecting the port to the antenna (Figure 4.2) [29].

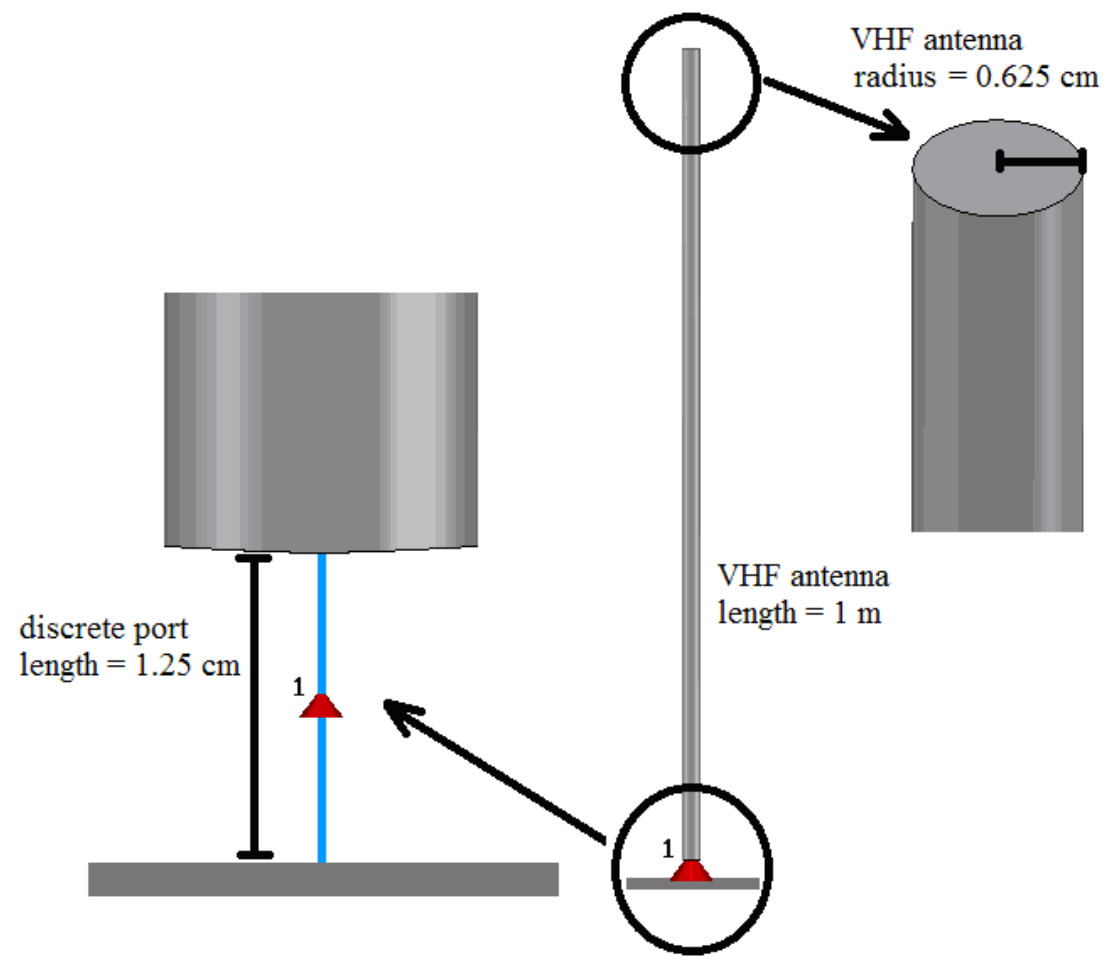

Figure 4.2 Dimensions of VHF antennas

- Since the aircraft platform and VHF antennas are metallic structures having high electrical conductivity, they are modeled with PEC (Perfect Electric Conductor) materials. PEC material, which is thought to exhibit infinite conductivity, does not exist in real life but it is an idealization. 
Using PEC material helps the simulations run faster than the simulations in where "real" materials with finite conductivity are used [26].

- Boundary of the problem space is defined as "open space” which operates as a free space. Electromagnetic waves can pass this boundary with minimal reflections.

- $\quad x-z$ plane is used as a symmetry plane since aircraft and VHF antennas are oriented symmetrically with respect to this plane. By symmetry property of problem, simulation time is reduced to its half.

\subsection{Modeling of Ship Platform and Its Antennas}

Ship platform and HF antennas placed on it are modeled as follows:

- Both ship platform and its antennas are modeled in a problem space defined as "normal” which operates as free space.

- Problem space in where the ship and its antennas are modeled is divided into about 200,000 hexahedral grid cells having smallest and largest side length of about $6 \mathrm{~cm}$ and $1 \mathrm{~m}$ respectively.

- Like the aircraft, ship is modeled by primitive geometric structures in order to reduce simulation complexity and simulation time, based on the real physical dimensions and geometrical structure of Patrol Boats in Turkish Naval Force Inventory.

- Ship has an overall length of $60 \mathrm{~m}$, width of $8 \mathrm{~m}$ and height (from sea level) of 15.5 m (Figure 4.3). 


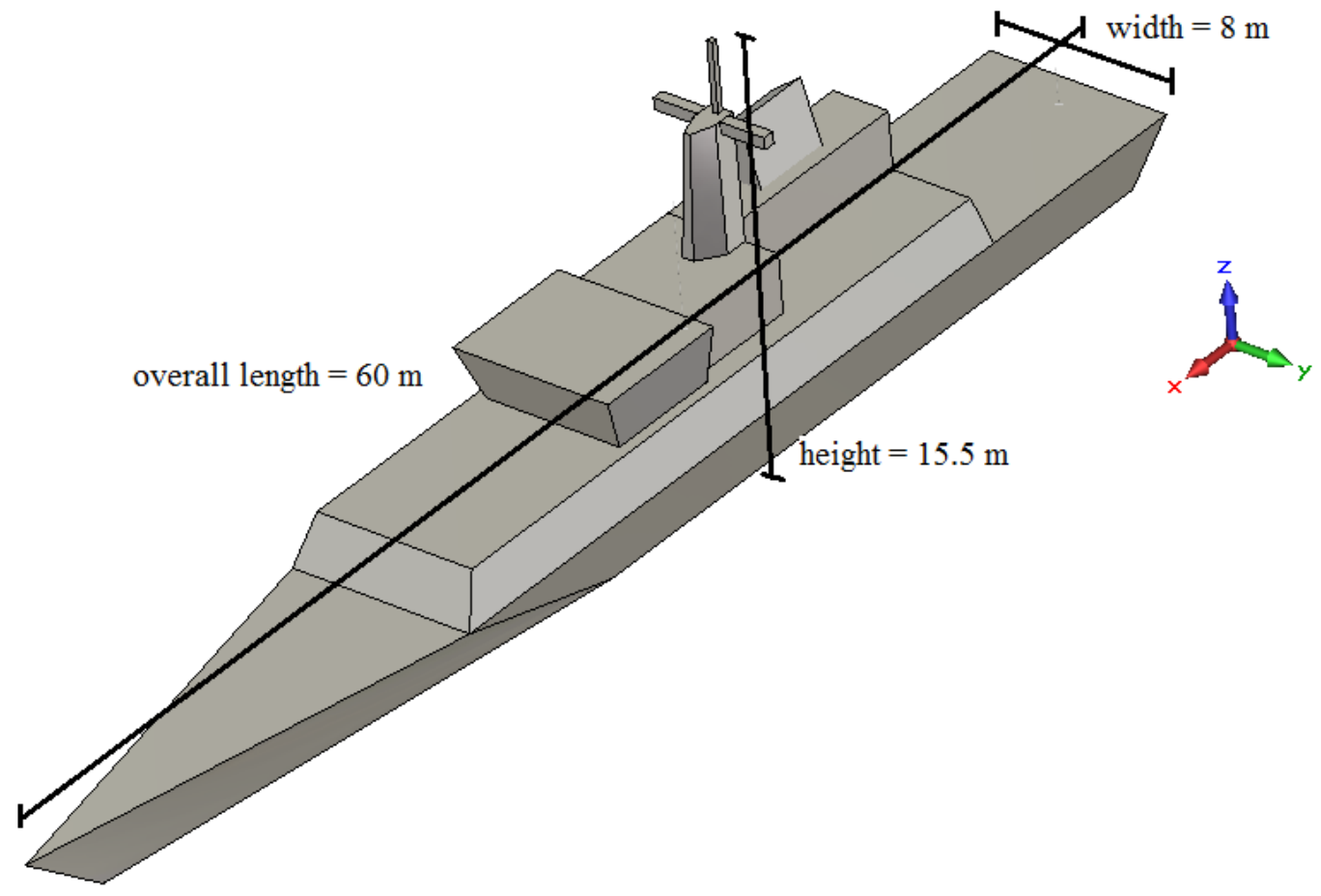

Figure 4.3 Dimensions of ship model

- HF antennas are modeled as $\lambda / 4$ monopole antennas operating in $2-30$ $\mathrm{MHz}$ frequency band. They have length of $8 \mathrm{~m}$ and radius of $2 \mathrm{~cm}$ (Figure 4.4).

- HF antennas are connected to ship and excited by discrete ports having length of $6 \mathrm{~cm}$ and impedance of $50 \Omega$ (Figure 4.4). 


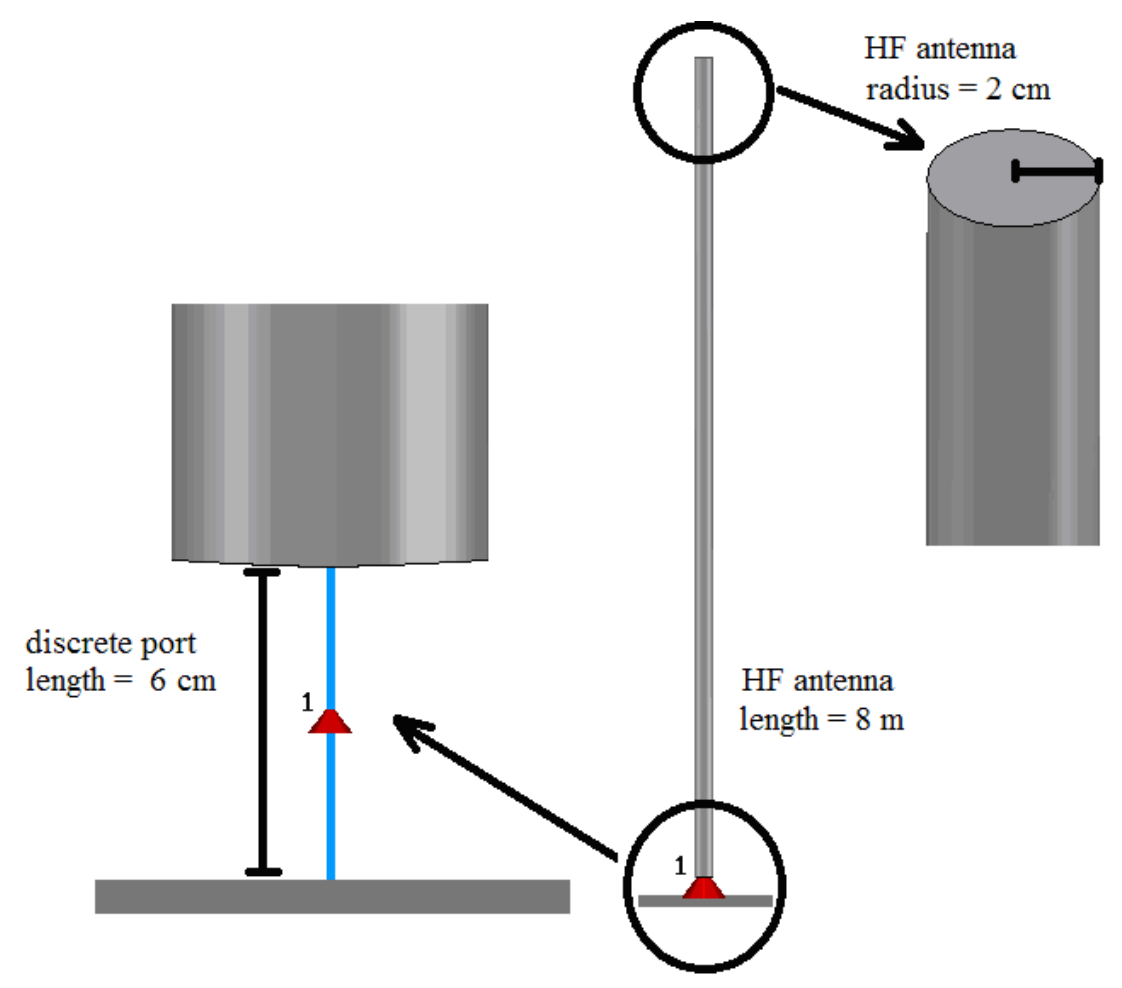

Figure 4.4 Dimensions of HF antennas

- Since the ship platform and HF antennas are metallic structures having high electrical conductivity, they are modeled with PEC (Perfect Electric Conductor) materials.

- Boundary of the problem space is defined as "open space” which operates as a free space. The boundary surface below the ship ( $z=0$ surface) is modeled with a material having conductivity of 5 Siemens / meter which has equal conductivity with sea water.

- Ship platform has a non-symmetric geometry with respect to $x-z$ plane because of the funnel on the right side of the weather deck. Unfortunately, $x-z$ plane is not used as a symmetry plane in contrast to what happened in aircraft case. 


\section{CHAPTER 5}

\section{SEARCH SPACES}

In general, there are finite and specific spaces on the platforms for the antennas to be mounted because of the structural and functional constraints. These constraints and "real-life applicability" concept are taken into account while defining the search spaces of antennas on aircraft and ship models in the simulations. In this chapter, the search spaces of VHF antennas on aircraft platform and the search spaces of HF antennas on ship platform are explained and illustrated in detail.

\subsection{Search Spaces of VHF Antennas on Aircraft Platform}

Three-dimensional Cartesian coordinate system is used in order to define the orientations of geometries with respect to origin. According to this system, the surface between fuselage and rear wings of the aircraft is assumed to be " $x=0 \mathrm{~m}$ surface” which is illustrated in Figure 5.3 and Figure 5.4. One of the VHF antennas is aimed to be placed on the upper fuselage, in the direction of $z$-axis. This antenna is named as "VHF upper antenna". The other VHF antenna is aimed to be placed on the lower fuselage in the direction of $(-z)-$ axis. This antenna is named as "VHF lower antenna".

Because of the symmetry requirement of the aircraft platform in real-life, search spaces of VHF antennas are defined on the centerlines of upper and lower fuselages and antennas are oriented with an angular separation of 180 degrees. 
Moreover, the orientation of antennas with an angular separation of 180 degrees is found to be advantageous by Davidson and Thiele, [30]. In their paper published in 1984, they showed how the coupling between two $\lambda / 4$ monopole antennas, mounted on metallic circular cylinder of radius $10 \lambda$, changes with the angular separation between antennas. They computed the coupling at $1 \mathrm{GHz}$ by two different techniques (IEMCAP and Hybrid Method of Moments techniques) in order to confirm the results. The circular cylinder and the monopole antenna models used in the computations are shown in Figure 5.1. The coupling results computed by two different techniques are given in Figure 5.2. According to this figure, the coupling between monopole antennas decreases as the angular separation increases. The minimum coupling occurs at an angular separation of 180 degrees. In their paper, Davidson and Thiele concluded that, on a circular metallic structure like a plane fuselage, minimum coupling between monopole antennas is achieved when the angular separation between them is 180 degrees.

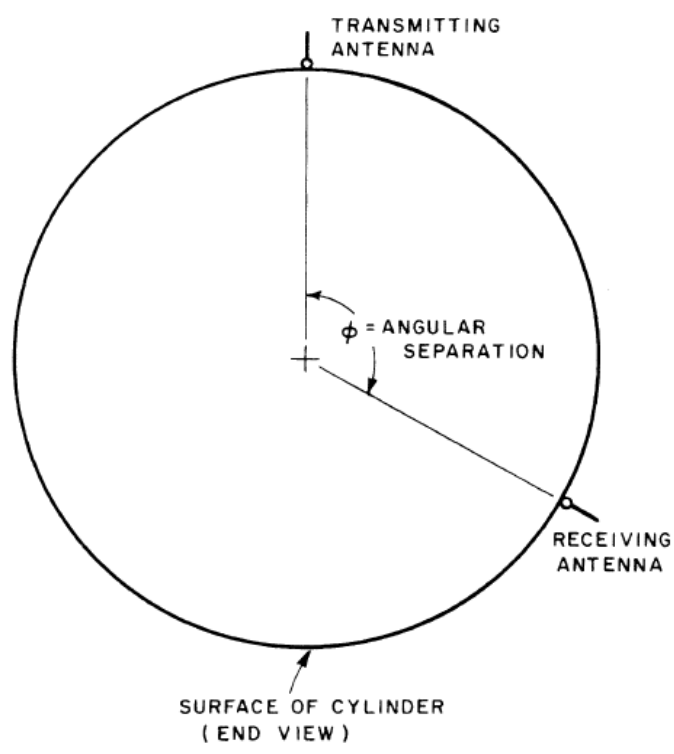

Figure 5.1 Two $\lambda / 4$ monopole antennas mounted on metallic circular cylinder of radius $10 \lambda[30]$ 


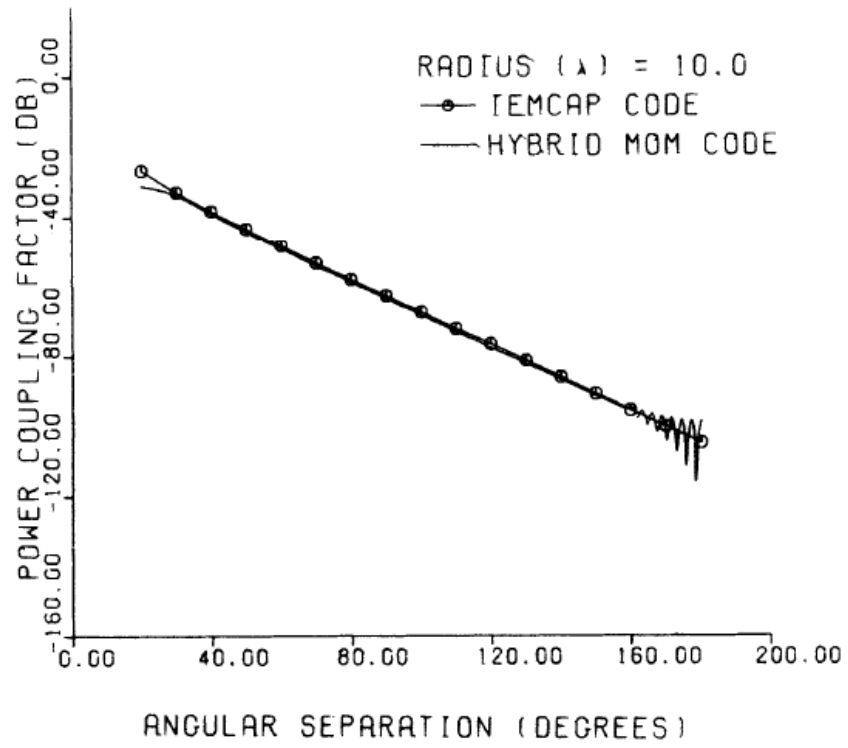

Figure 5.2 Coupling between monopole antennas vs. angular separation [30]

The search space of VHF upper antenna is defined on the centerline of upper fuselage between $x=0 \mathrm{~m}$ and $x=9 \mathrm{~m}$ (Figure 5.3). The search space of VHF lower antenna is defined on the centerline of lower fuselage between $x=7 \mathrm{~m}$ and $x=14.5 \mathrm{~m}$ (Figure 5.4). The space between $x=0 \mathrm{~m}$ and $x=7 \mathrm{~m}$ on the lower fuselage is not included in the search space of VHF lower antenna since fuel tank, ordnance and wheels of aircraft are located on that space in real-life. 


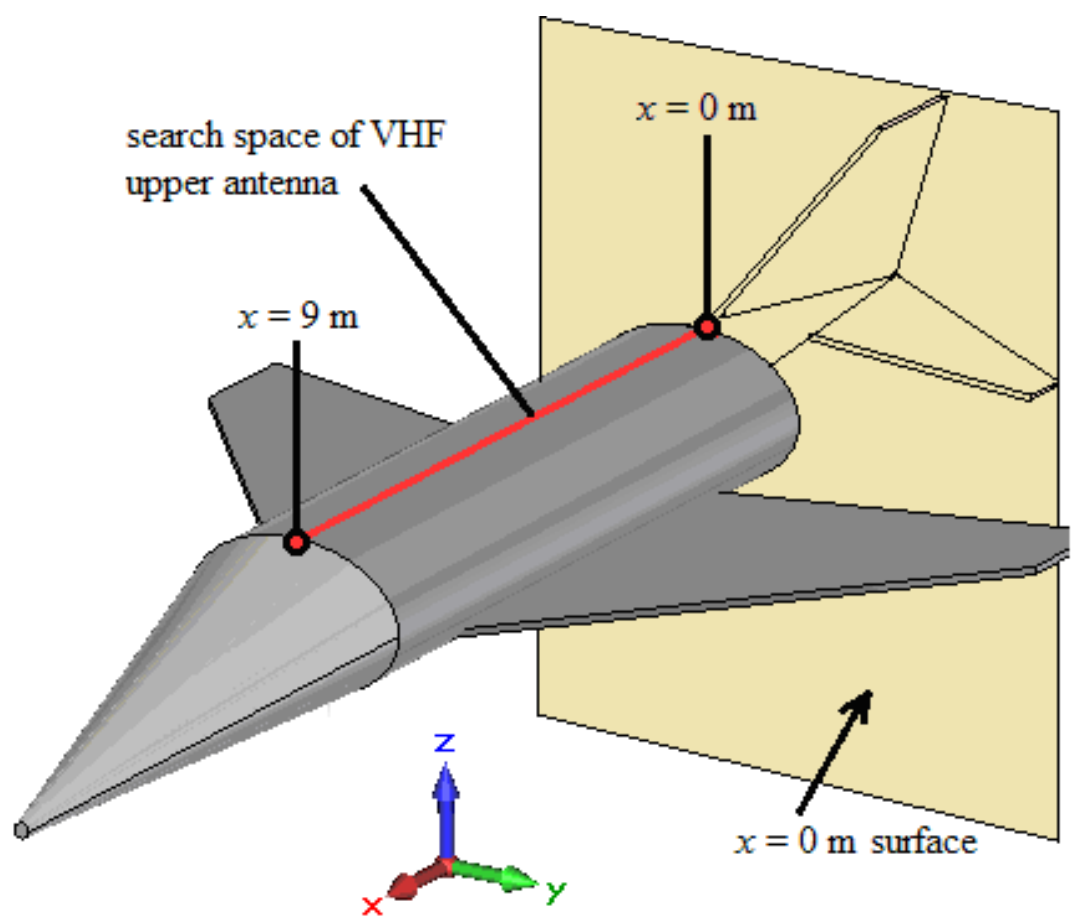

Figure 5.3 The search space of VHF upper antenna

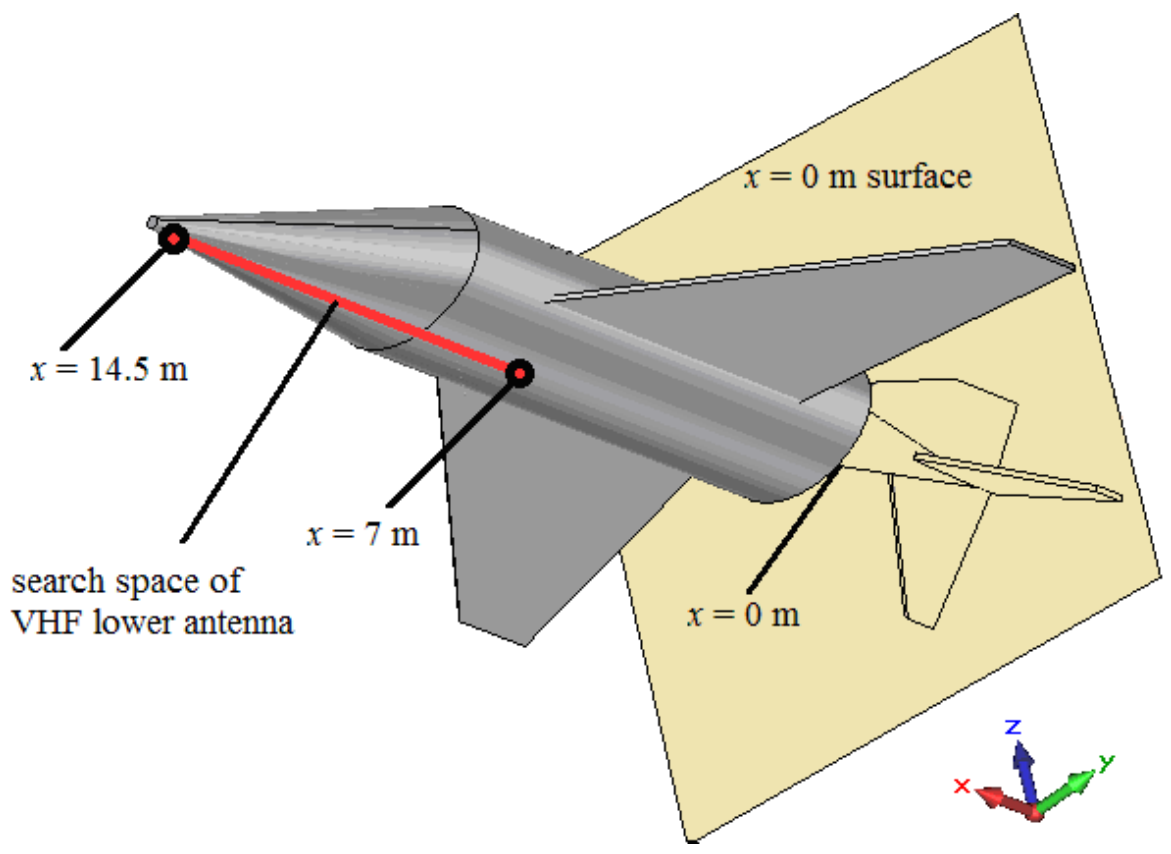

Figure 5.4 The search space of VHF lower antenna 


\subsection{Search Spaces of HF Antennas on Aircraft Platform}

One of the HF antennas is aimed to be placed on the bridge, in the direction of $z-$ axis. This antenna is named as "HF fore antenna". The other HF antenna is aimed to be placed on the stern, in the direction of $z$ - axis. This antenna is named as "HF aft antenna”.

Different from aircraft antennas, HF antennas have two-dimensional search spaces as shown in Figure 5.5.The search space of HF fore antenna has an area of $42 \mathrm{~m}^{2}$ ( $6 \mathrm{~m} \times 7 \mathrm{~m}$ ) on the $z=7.5 \mathrm{~m}$ surface and the search space of HF aft antenna has an area of $60 \mathrm{~m}^{2}(7.5 \mathrm{~m} \times 8 \mathrm{~m})$ on the $z=3 \mathrm{~m}$ surface. Coordinates of search spaces of both antennas are given in detail in Figure 5.5.

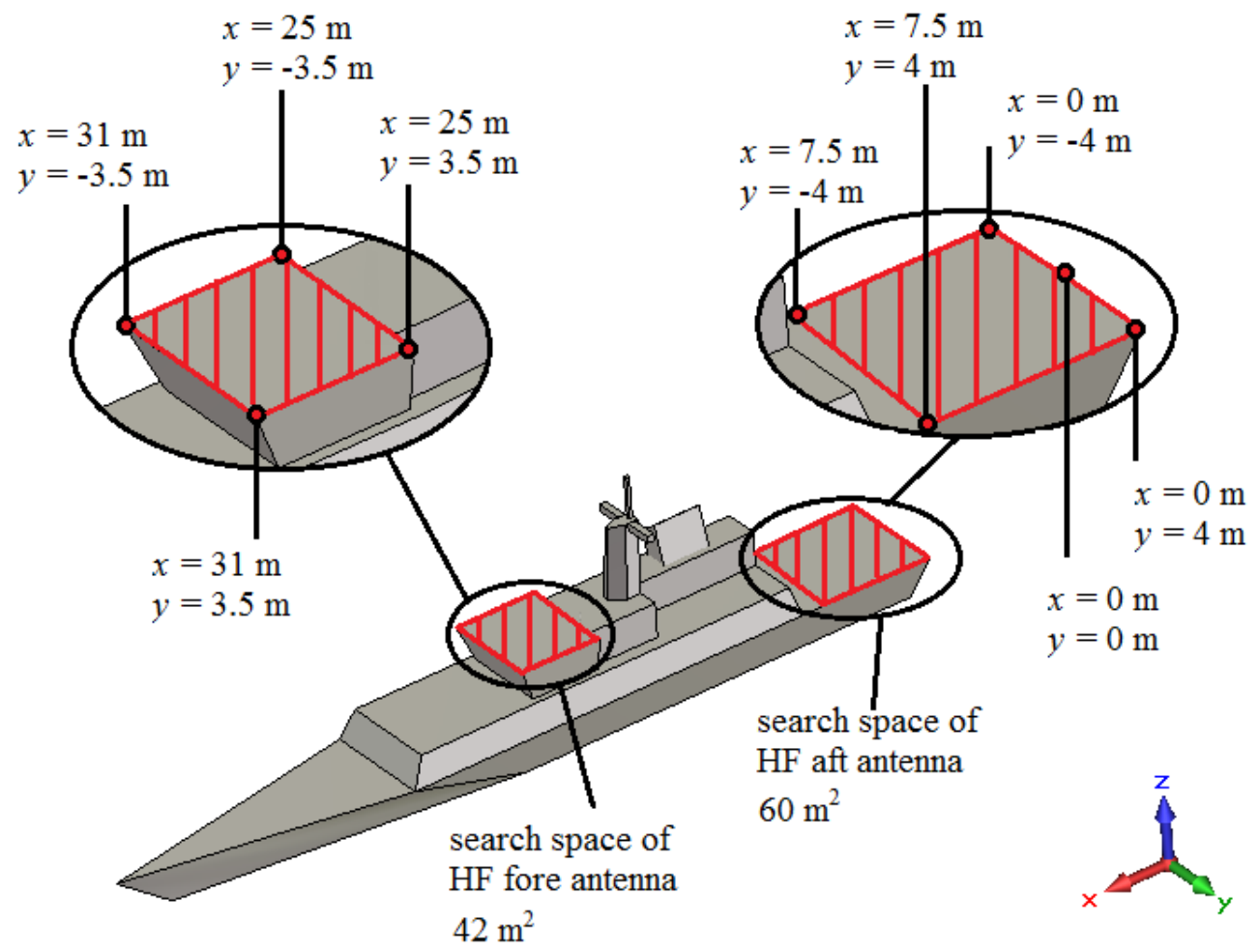

Figure 5.5 Coordinates of search spaces of HF fore and HF aft antennas 


\section{CHAPTER 6}

\section{OPTIMIZATION OF ANTENNA PLACEMENTS}

Optimizations of antenna placements on aircraft and ship are performed by using optimization toolbox of CST - MWS. At each simulation, placements of the antennas are changed among their search spaces according to the search strategy of optimization algorithm used. For each placement, “coordinates of the antennas" are regarded as a potential solution to the optimization problem. Therefore, coordinates of two antennas, in pair, are considered as a "chromosome" in genetic algorithm optimization and as a "particle” in particle swarm optimization.

Moreover; for each of the placements, the coupling between antennas is calculated for whole frequency band of operation of the antennas. After that, the coupling between antennas is averaged over whole frequency band of operation and this "average coupling value" is considered as the "fitness value" of that antenna placement (fitness of chromosome or particle). By averaging the coupling over the whole frequency band of operation of the antennas, effect of the coupling at each frequency in that band is considered in the optimizations. In aircraft antenna placement optimizations, the coupling is calculated and averaged among 30-88 $\mathrm{MHz}$ band and in ship antenna placement optimizations, the coupling is calculated and averaged among 2-30 MHz band. It is important to note that, since there is no active element like antenna tuning unit, active filter, etc. connected to the antennas, coupling between them is reciprocal. 
The aim of both optimization techniques is to find an antenna placement, which minimizes the average coupling between antennas at each platform by using the inputs explained above.

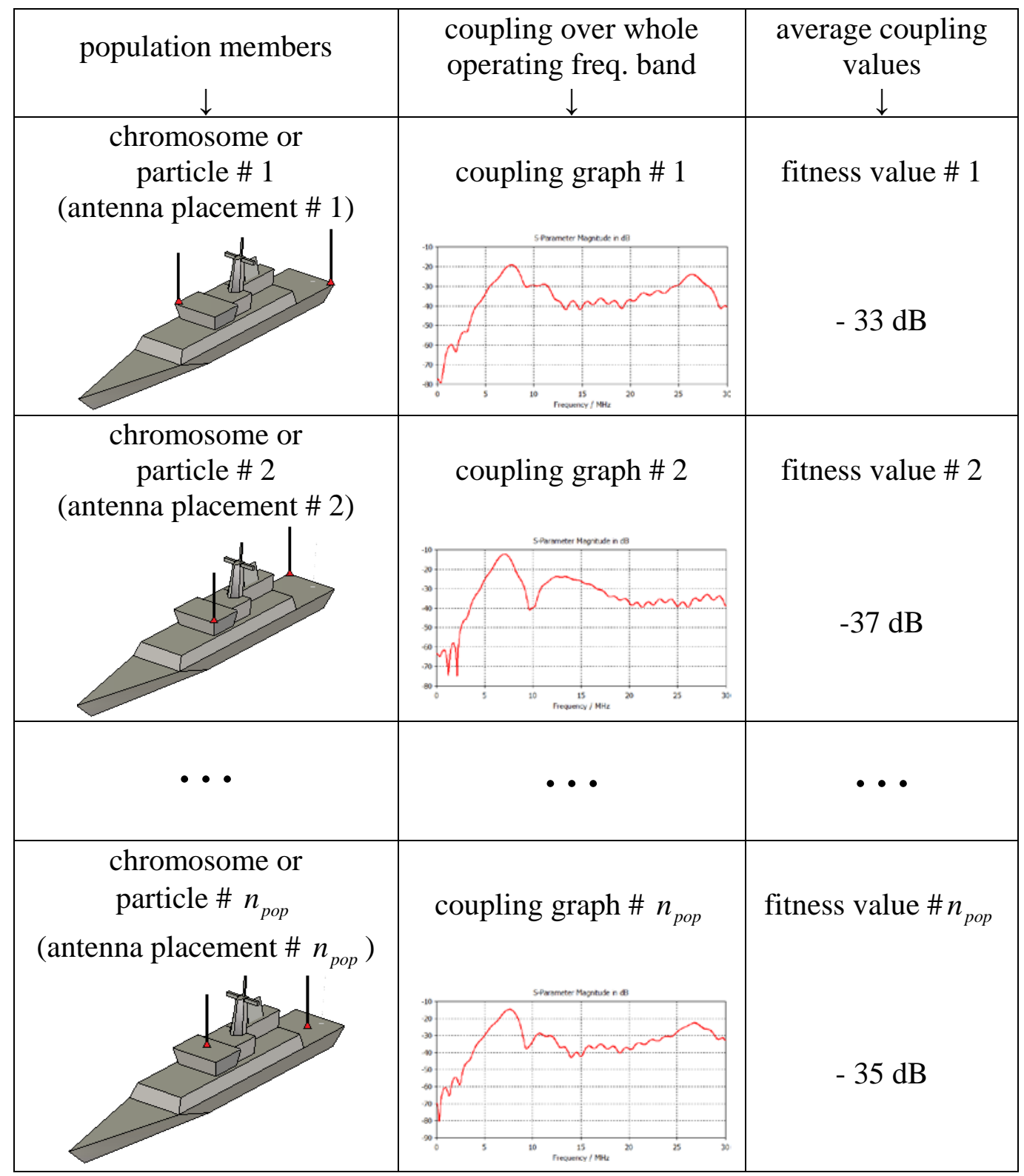

Figure 6.1 Example showing how the simulation results are introduced as optimization parameters 
An example showing how "the antenna placements" and "average coupling values” are introduced as optimization parameters into optimization algorithms is given in Figure 6.1. In the first column, different antenna placements which are regarded as chromosomes or particles are given. In the second column, graphs showing corresponding coupling values versus operating frequency band of the antennas are given. Finally, in the third column, corresponding fitness values (average coupling values) of the chromosomes / particles are given. In this figure, each row represents a simulation and $n_{\text {pop }}$ represents the size of the population.

It is important to note that; coordinates of the antennas at their optimal locations are rounded to two decimal places in the tables below for simplicity.

\subsection{Optimization of Antenna Placement on Aircraft}

\subsubsection{Optimization of Antenna Placement on Aircraft by Genetic Algorithm Optimization}

Parameters of the genetic algorithm optimization are set as follows in optimization of antenna placement on aircraft:

- Search spaces of VHF antennas are defined on the centerlines of upper and lower fuselages of the aircraft and both antennas move along only on the $x$ - axis. Therefore, the coordinates of both antennas on the $x$ - axis are defined as variables. The number of variables is 2 .

- Number of chromosomes in the population is defined as 40 .

- Two different random creation functions which are based on "uniform random distribution" and "Latin hypercube distribution” are used for the generation of the initial population of chromosomes. 
- Maximum number of iterations is defined as 30. Algorithm stops when maximum number of iterations is achieved.

- Total number of simulations is calculated by multiplying "the number of chromosomes in the population" and "the maximum number of iterations". However; according to selection strategy of the algorithm used, half of the population which is composed of the fittest individuals is "directly transferred" to the next generation as parents. Therefore, total number of simulations becomes $(40 / 2) \times(30+1)=620$ instead of $40 \times 30=1200$.

- CST - MWS genetic algorithm optimizer uses variable crossover rate which is adopted according to the genetic structure of chromosomes at each iteration (explained in Section 2.1 in details).

- For each population generated by two different distribution techniques, three different optimization runs with mutation rates of $60 \%, 30 \%$ and 1 $\%$ are operated. Therefore, six different antenna placements are determined by six optimization runs having different optimization parameters.

Coordinates of VHF antennas on the aircraft and final average coupling values between them obtained by different optimization runs are given in Table 6.1. 
Table 6.1 Antenna placement results on aircraft obtained by genetic algorithm optimization

\begin{tabular}{|c|c|c|c|c|}
\hline $\begin{array}{c}\text { Distribution } \\
\text { Technique Used } \\
\text { for Generation of } \\
\text { Initial Population }\end{array}$ & $\begin{array}{c}\text { Mutation } \\
\text { Rates }\end{array}$ & $\begin{array}{c}\text { Coordinate } \\
\text { of VHF } \\
\text { Upper } \\
\text { Antenna }\end{array}$ & $\begin{array}{c}\text { Coordinate } \\
\text { of VHF } \\
\text { Lower } \\
\text { Antenna }\end{array}$ & $\begin{array}{c}\text { Average } \\
\text { Coupling } \\
\text { Between } \\
\text { Antennas }\end{array}$ \\
\hline $\begin{array}{c}\text { Uniform Random } \\
\text { Distribution }\end{array}$ & $60 \%$ & $x=0.48 \mathrm{~m}$ & $x=8.28 \mathrm{~m}$ & $-44.62 \mathrm{~dB}$ \\
\cline { 2 - 5 } & $30 \%$ & $x=0.86 \mathrm{~m}$ & $x=7.93 \mathrm{~m}$ & $-44.47 \mathrm{~dB}$ \\
\hline \multirow{2}{*}{$\begin{array}{c}\text { Latin Hypercube } \\
\text { Distribution }\end{array}$} & $60 \%$ & $x=0.93 \mathrm{~m}$ & $x=9.92 \mathrm{~m}$ & $-44.15 \mathrm{~dB}$ \\
\cline { 2 - 5 } & $1 \%$ & $x=0.86 \mathrm{~m}$ & $x=7.88 \mathrm{~m}$ & $-45.03 \mathrm{~dB}$ \\
\cline { 2 - 5 } & & $x=1.07 \mathrm{~m}$ & $x=7.87 \mathrm{~m}$ & $-44.95 \mathrm{~dB}$ \\
\hline
\end{tabular}

During these six different optimization runs;

- "minimum" of the average coupling values of the individuals generated at each iteration

- "maximum" of the average coupling values of the individuals generated at each iteration

- "population average" of the average coupling values of the individuals generated at each iteration

are calculated in order to monitor the progresses and performances of the these optimization runs. Graphs related to these calculations are given in Figure 6.2 through Figure 6.7. 


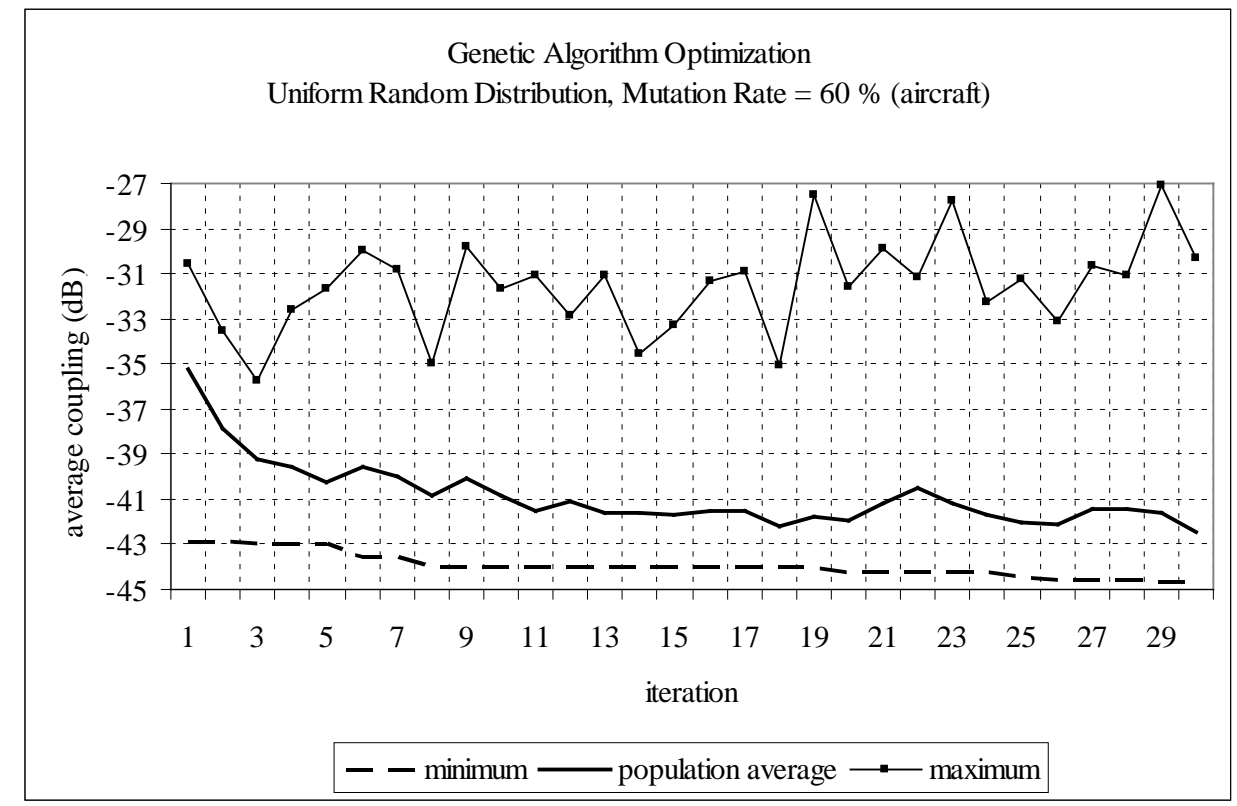

Figure 6.2 Progress of the optimization run with an initial population generated by uniform random distribution technique and with a mutation rate of $60 \%$

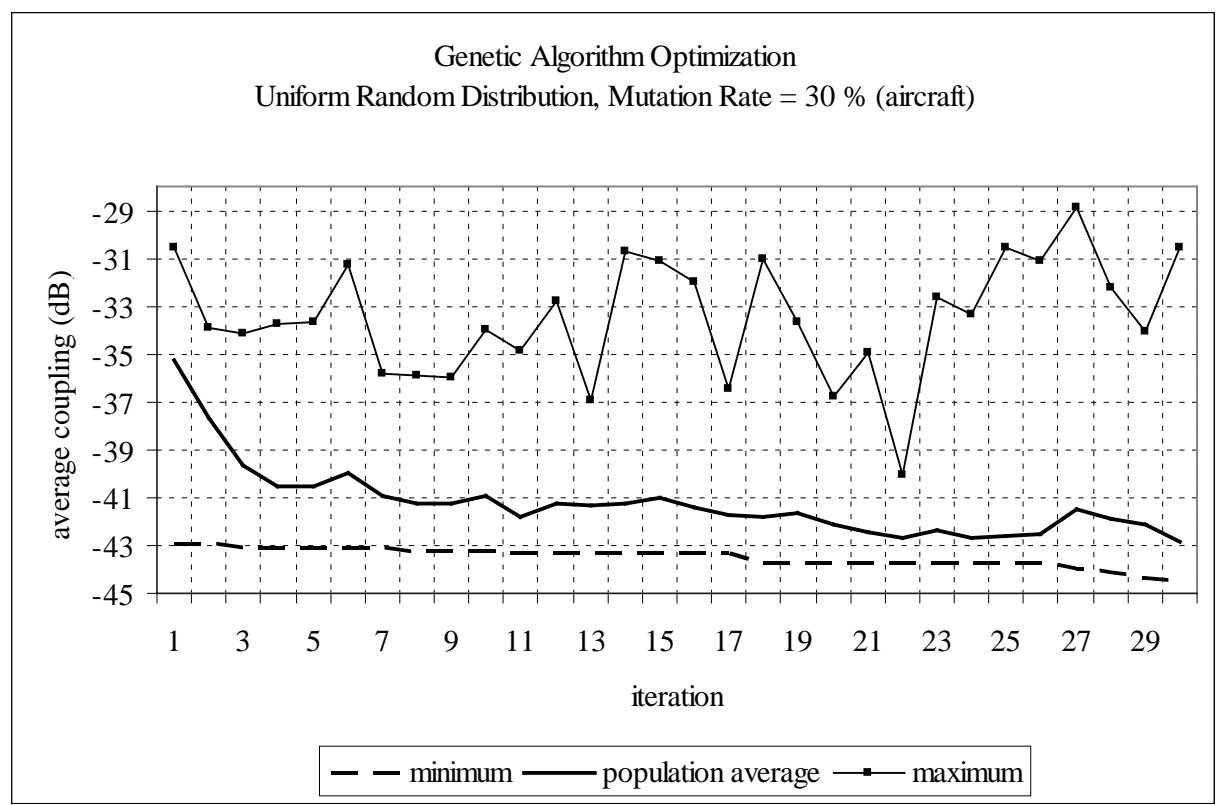

Figure 6.3 Progress of the optimization run with an initial population generated by uniform random distribution technique and with a mutation rate of $30 \%$ 


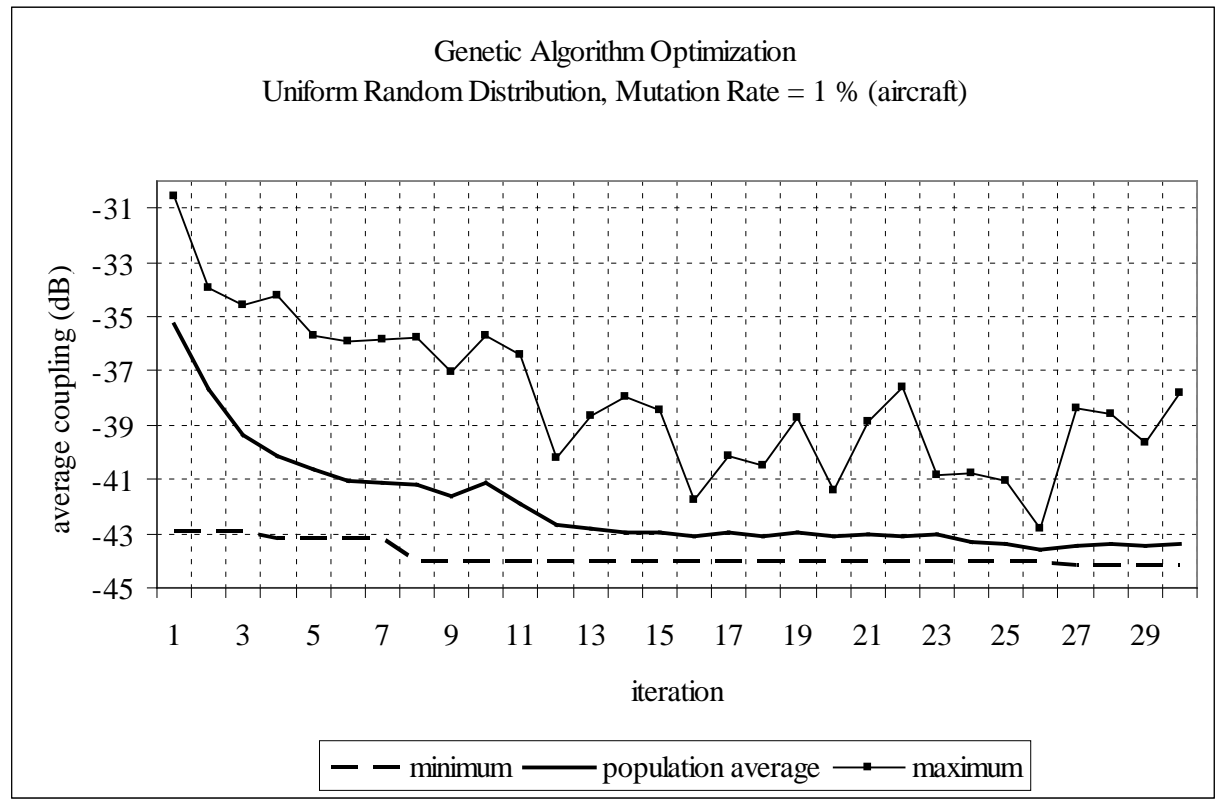

Figure 6.4 Progress of the optimization run with an initial population generated by uniform random distribution technique and with a mutation rate of $1 \%$

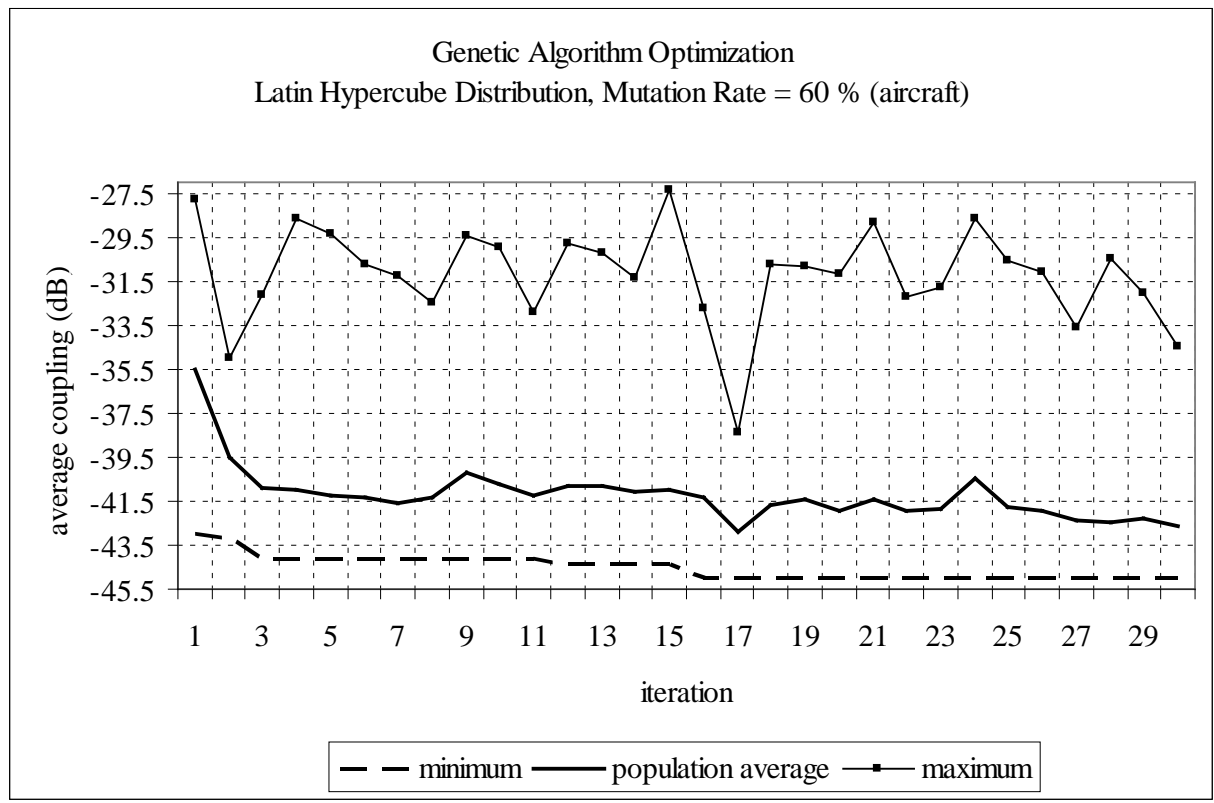

Figure 6.5 Progress of the optimization run with an initial population generated by Latin hypercube distribution technique and with a mutation rate of $60 \%$ 


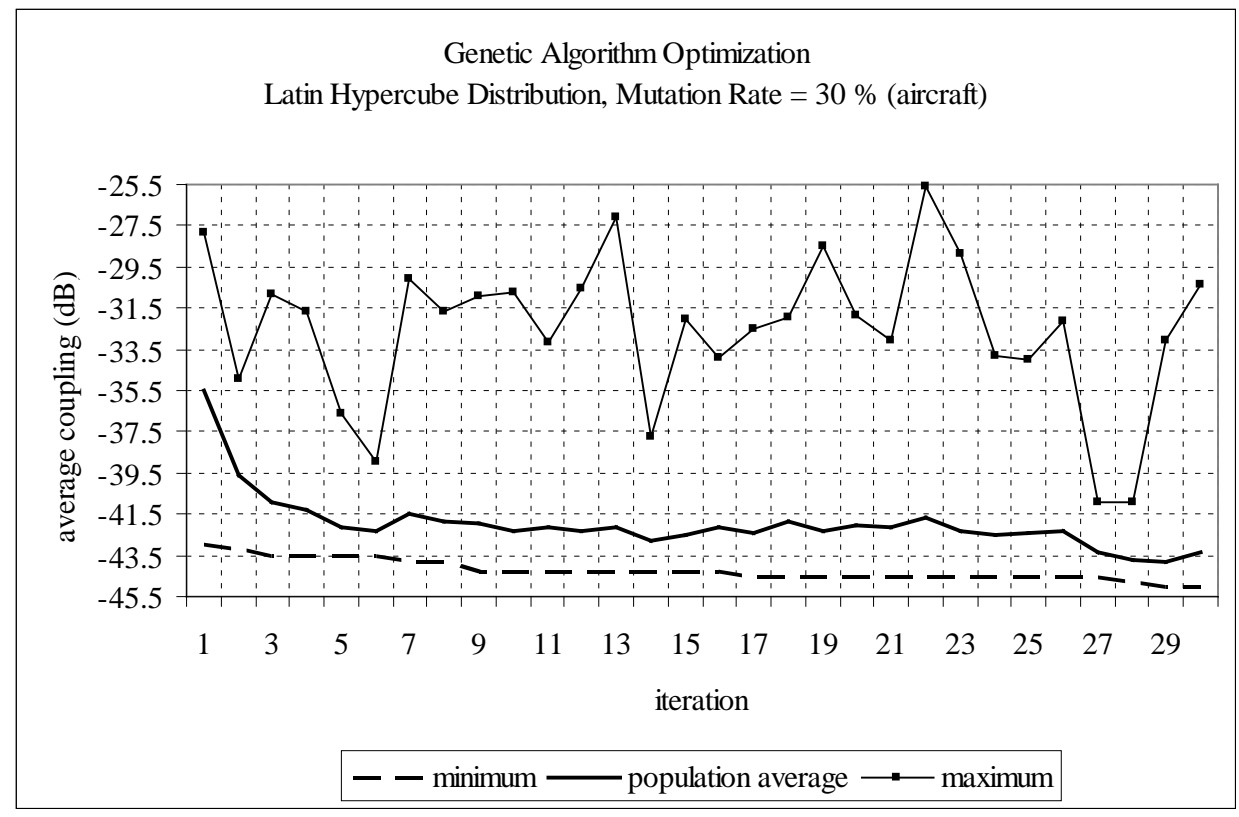

Figure 6.6 Progress of the optimization run with an initial population generated by Latin hypercube distribution technique and with a mutation rate of $30 \%$

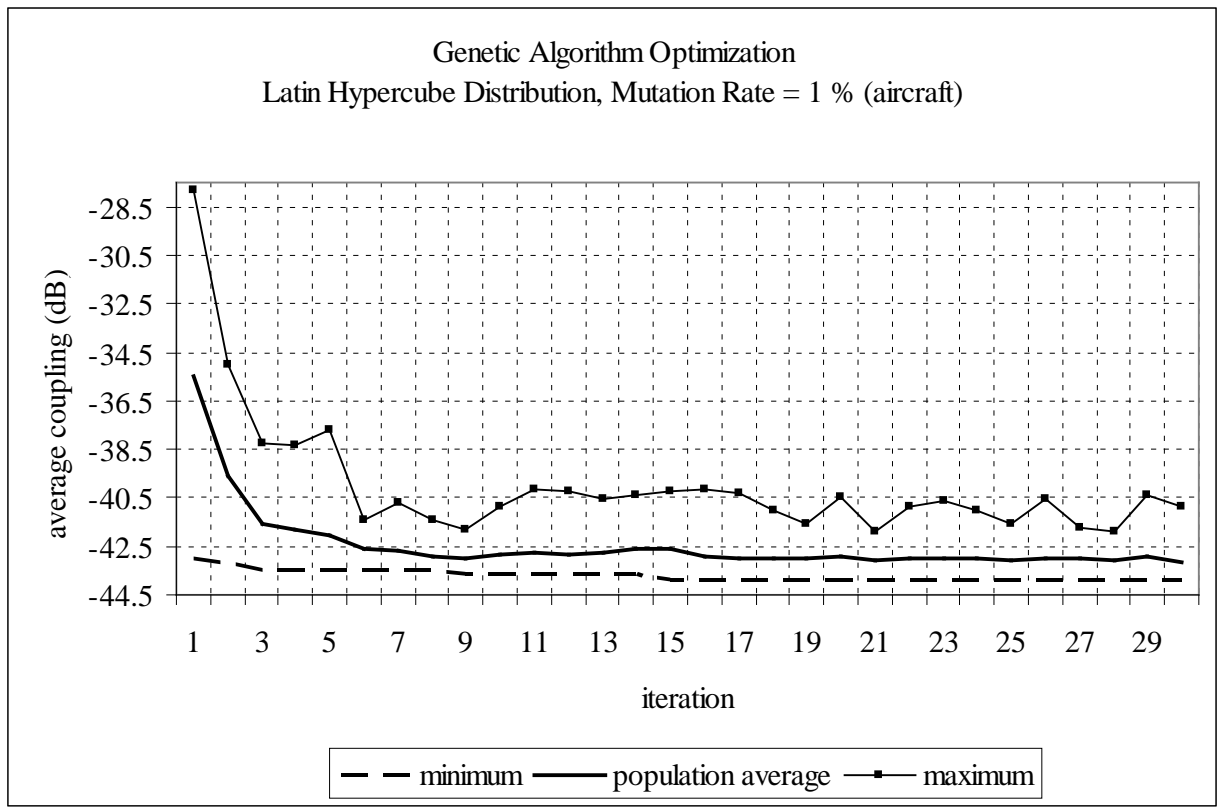

Figure 6.7 Progress of the optimization run with an initial population generated by Latin hypercube distribution technique and with a mutation rate of $1 \%$ 
According to Table 6.1 and Figure 6.2 through Figure 6.7:

- For all of the six antenna placements in Table 6.1, final average coupling values are very close to each other.

- Minimum (best) final average coupling between the antennas, - $45.03 \mathrm{~dB}$, is achieved in the optimization run in where the initial population is generated by Latin hypercube distribution technique and mutation rate of $60 \%$ is used.

- Maximum (worst) final average coupling between the antennas, - 43.85 $\mathrm{dB}$, occurs in the optimization run in where the initial population is generated by Latin hypercube distribution technique and mutation rate of 1 $\%$ is used.

- Coordinates of antennas in six different antenna placements also are very close to each other like the final average coupling values.

- The placement; where VHF upper antenna is located at $x=0.86 \mathrm{~m}$ and VHF lower antenna is located at $x=7.88 \mathrm{~m}$, yields minimum final average coupling between the antennas. This placement is illustrated in Figure 6.8. 


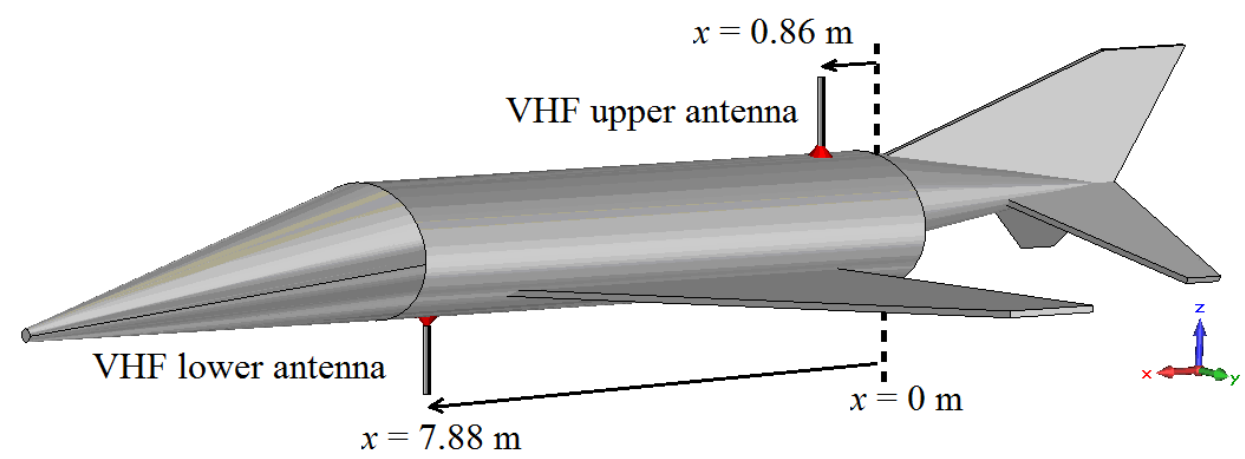

Figure 6.8 The placement yielding minimum final average coupling between VHF antennas (genetic algorithm optimization)

- As it is seen in graphs above, population average of the average coupling values and minimum average coupling values decrease as the iteration number increases. In parallel with Holland's idea [12], individuals generated at each new iteration are generally fitter than their parents. This downward trend in the population average of the average coupling values may be thought as a marker of achievement of the global optimum.

- It is important to note that, in the optimization runs with mutation rates of $60 \%$ and $30 \%$, the population average of the average coupling values and the maximum average coupling values fluctuate through the iterations. Because of these fluctuations, the difference between the minimum and the maximum average coupling curves reaches up to $20 \mathrm{~dB}$. The reason for these fluctuations is the high diversity in the population because of high mutation rates. These fluctuations and the difference between the minimum and the maximum average coupling curves become smaller as the mutation rate decreases. 
- Each of the genetic algorithm optimization runs, namely 620 simulations, last for about 26 hours (about 1 day) with a machine having 4 GB random access memory and $2.53 \mathrm{GHz}$ Intel ${ }^{\circledR}$ Core $^{\mathrm{TM}} 2$ Duo central processor unit.

\subsubsection{Optimization of Antenna Placement on Aircraft by Particle Swarm Optimization}

Parameters of the particle swarm optimization are set as follows in optimization of antenna placement on aircraft:

- The coordinates of VHF antennas on the $x$ - axis are defined as variables. Therefore, variable number is defined as 2 .

- Number of particles in the swarm is defined as 40 .

- Two different random creation functions which are based on "uniform random distribution" and "Latin hypercube distribution” are used for the generation of the initial swarm of particles.

- Maximum number of iterations is defined as 30. Algorithm stops when maximum number of iterations is reached.

- Total number of simulations is calculated by multiplying "the number of particles in the swarm" and "the maximum number of iterations". Therefore, total number of simulations becomes $40 \times 30=1200$.

- CST-MWS particle swarm optimizer randomly determines the weights of parameters of position and velocity vectors (explained in Section 2.2 in detail). 
- Two different antenna placements are determined for each of the swarms generated by two different distribution techniques.

Coordinates of VHF antennas on the aircraft and final average coupling values between them obtained by different optimization runs are given in Table 6.2.

Table 6.2 Antenna placement results on aircraft obtained by particle swarm optimization

\begin{tabular}{|c|c|c|c|}
\hline $\begin{array}{c}\text { Distribution } \\
\text { Gechnique Used for } \\
\text { Swarm }\end{array}$ & $\begin{array}{c}\text { Coordinate } \\
\text { of VHF } \\
\text { Upper } \\
\text { Antenna }\end{array}$ & $\begin{array}{c}\text { Coordinate } \\
\text { of VHF } \\
\text { Lower } \\
\text { Antenna }\end{array}$ & $\begin{array}{c}\text { Average } \\
\text { Coupling } \\
\text { Between } \\
\text { Antennas }\end{array}$ \\
\hline $\begin{array}{c}\text { Uniform Random } \\
\text { Distribution }\end{array}$ & $x=0.84 \mathrm{~m}$ & $x=7.88 \mathrm{~m}$ & $-44.91 \mathrm{~dB}$ \\
\hline $\begin{array}{c}\text { Latin Hypercube } \\
\text { Distribution }\end{array}$ & $x=0.81 \mathrm{~m}$ & $x=7.93 \mathrm{~m}$ & $-44.81 \mathrm{~dB}$ \\
\hline
\end{tabular}

During these two different optimization runs;

- "minimum" of the average coupling values of the individuals generated at each iteration

- maximum" of the average coupling values of the individuals generated at each iteration

- "swarm average" of the average coupling values of the individuals generated at each iteration

are calculated in order to monitor the progresses and performances of the these optimization runs. Graphs related to these calculations are given in Figure 6.9 and Figure 6.10. 


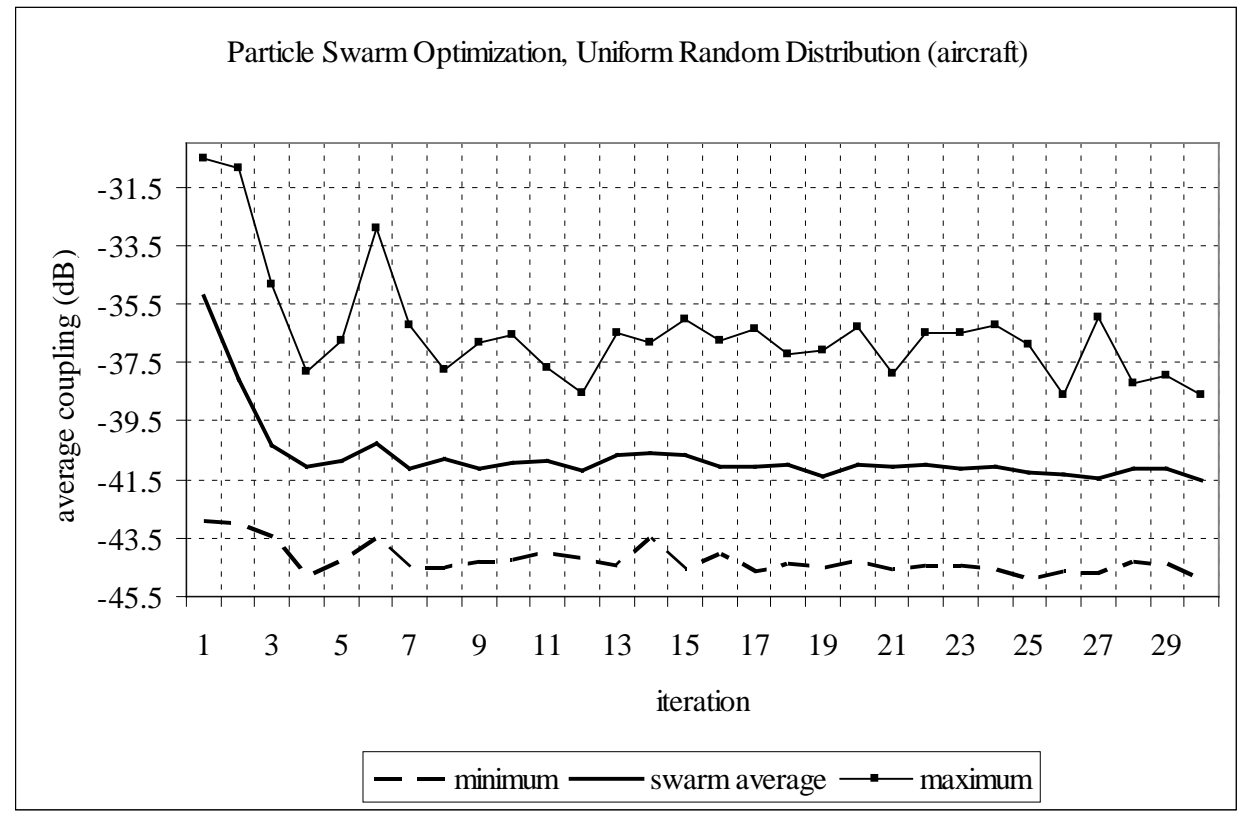

Figure 6.9 Progress of the optimization run with an initial swarm generated by uniform random distribution technique

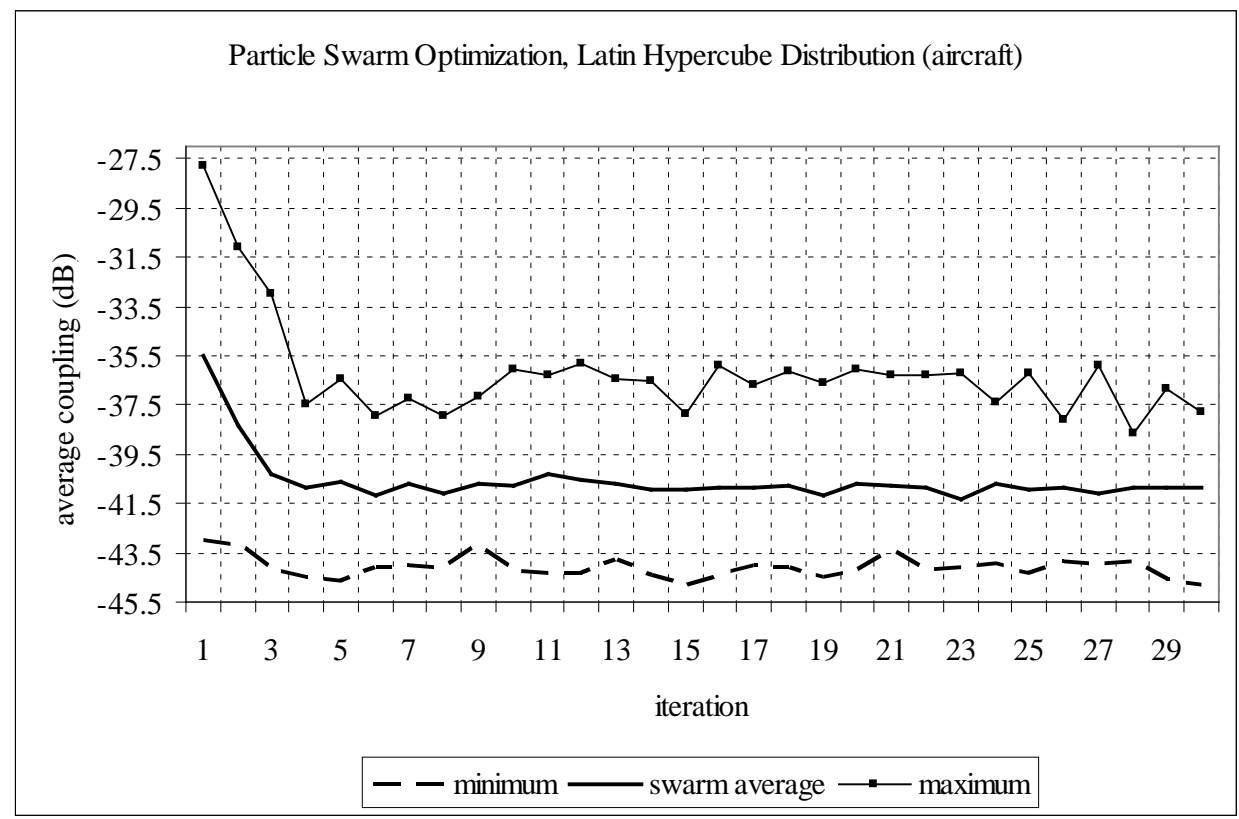

Figure 6.10 Progress of the optimization run with an initial swarm generated by Latin hypercube distribution technique 
According to Table 6.2, Figure 6.9 and Figure 6.10:

- For both of the antenna placements in Table 6.2, final average coupling values are very close to each other.

- Minimum (best) final average coupling between the antennas, - $44.91 \mathrm{~dB}$, is achieved in the optimization run in where the initial swarm is generated by uniform random distribution technique.

- Coordinates of antennas in two different antenna placements also are very close to each other like the final average coupling values.

- The placement; where VHF upper antenna is located at $x=0.84 \mathrm{~m}$ and VHF lower antenna is located at $x=7.88 \mathrm{~m}$, yields minimum final average coupling between the antennas. This placement is illustrated in Figure 6.11 .

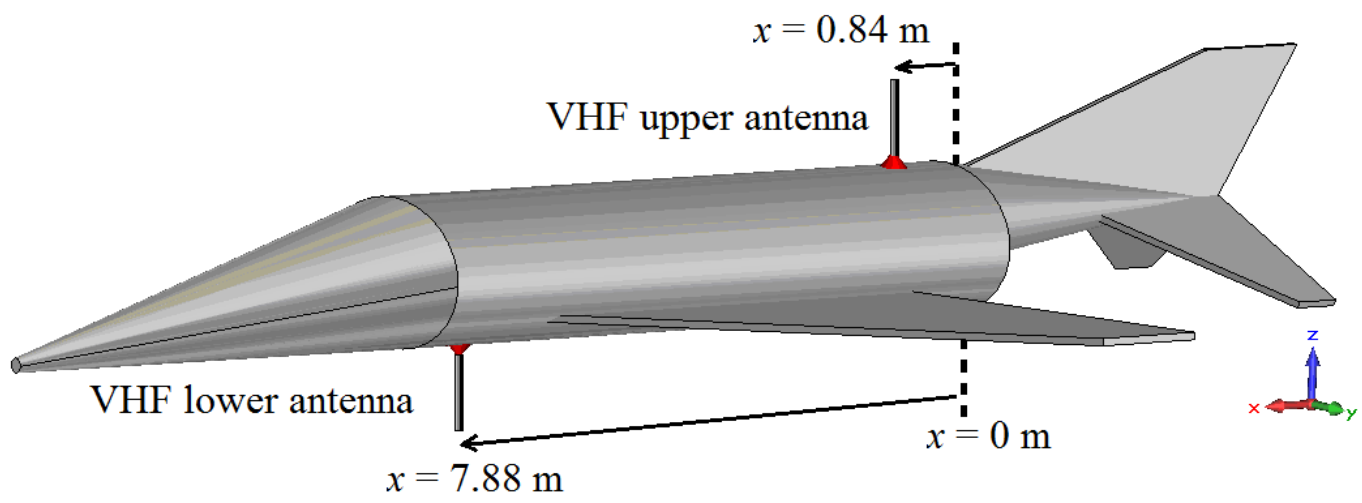

Figure 6.11 The placement yielding minimum final average coupling between VHF antennas (particle swarm optimization) 
- As it is seen in Figure 6.9 and Figure 6.10, swarm average of the average coupling values and minimum average coupling values decrease as the iteration number increases. This downward trend in the swarm average of the average coupling values may be thought as a marker of tendency of the whole swarm towards the global optimum.

- The fluctuations in the swarm average of the average coupling values and the maximum average coupling values are very small in contrast to the fluctuations in the genetic algorithm optimization runs with mutation rates of $60 \%$ and $30 \%$. Moreover, the difference between the minimum and the maximum average coupling values are smaller when compared with the differences in the genetic algorithm optimization runs with mutation rates of $60 \%$ and $30 \%$. Since there is no mutation operator in particle swarm optimization, these fluctuations and the difference between the curves resemble the fluctuations and the differences in the genetic algorithm optimization runs with mutation rate of $1 \%$.

- Each of the particle swarm optimization runs, namely 1200 simulations, last for about 50 hours (about 2 days) with a machine having 4 GB random access memory and $2.53 \mathrm{GHz}$ Intel ${ }^{\circledR}$ Core $^{\mathrm{TM}} 2$ Duo central processor unit.

\subsection{Optimization of Antenna Placement on Ship}

\subsubsection{Optimization of Antenna Placement on Ship by Genetic Algorithm Optimization}

Parameters of the genetic algorithm optimization are set as follows in optimization of antenna placement on ship: 
- Different from aircraft antennas, HF antennas on ship have twodimensional search spaces. Coordinates of the HF antennas on $x$ and $y$ axes are defined as variables. Therefore, variable number is defined as 4 .

- Number of chromosomes in the population is defined as 40 .

- Two different random creation functions which are based on "uniform random distribution” and "Latin hypercube distribution” are used for the generation of the initial population of chromosomes.

- Maximum number of iterations is defined as 30. Algorithm stops when maximum number of iterations is reached.

- Total number of simulations is $(40 / 2) \times(30+1)=620$ as in the aircraft antenna placement optimization case.

- CST- MWS genetic algorithm optimizer uses variable crossover rate which is adopted according to the genetic structure of chromosomes at each iteration (explained in Section 2.1 in detail).

- For each population generated by two different distribution techniques, three different optimization runs with mutation rates of $60 \%, 30 \%$ and 1 $\%$ are operated. Therefore, six different antenna placements are determined by six optimization runs having different optimization parameters.

Coordinates of HF antennas on the ship and final average coupling values between them obtained by different optimization runs are given in Table 6.3. 
Table 6.3 Antenna placement results on ship obtained by genetic algorithm optimization

\begin{tabular}{|c|c|c|c|c|}
\hline $\begin{array}{c}\text { Distribution } \\
\text { Technique Used } \\
\text { for Generation of } \\
\text { Initial Population }\end{array}$ & $\begin{array}{c}\text { Mutation } \\
\text { Rates }\end{array}$ & $\begin{array}{c}\text { Coordinate } \\
\text { of HF Fore } \\
\text { Antenna }\end{array}$ & $\begin{array}{c}\text { Coordinate } \\
\text { of HF Aft } \\
\text { Antenna }\end{array}$ & $\begin{array}{c}\text { Average } \\
\text { Coupling } \\
\text { Between } \\
\text { Antennas }\end{array}$ \\
\hline \multirow{2}{*}{$\begin{array}{c}\text { Uniform Random } \\
\text { Distribution }\end{array}$} & $30 \%$ & $\begin{array}{c}x=25.9 \mathrm{~m} \\
y=3.3 \mathrm{~m}\end{array}$ & $\begin{array}{c}x=1.1 \mathrm{~m} \\
y=0.5 \mathrm{~m}\end{array}$ & $-38.27 \mathrm{~dB}$ \\
\cline { 2 - 5 } & $1 \%$ & $\begin{array}{c}x=25.6 \mathrm{~m} \\
y=2.7 \mathrm{~m}\end{array}$ & $\begin{array}{c}x=1.5 \mathrm{~m} \\
y=0.3 \mathrm{~m}\end{array}$ & $-38.09 \mathrm{~dB}$ \\
\hline \multirow{2}{*}{$\begin{array}{c}\text { Latin Hypercube } \\
\text { Distribution }\end{array}$} & $30 \%$ & $x=26.2 \mathrm{~m}$ & $x=1.2 \mathrm{~m}$ & $-38.22 \mathrm{~dB}$ \\
\cline { 2 - 6 } & $1 \%$ & $y=2.7 \mathrm{~m}$ & $y=0.1 \mathrm{~m}$ & $-37.66 \mathrm{~dB}$ \\
\cline { 2 - 6 } & $1 \%$ & $x=26.7 \mathrm{~m}$ & $x=1.7 \mathrm{~m}$ & $-38.16 \mathrm{~dB}$ \\
\hline
\end{tabular}

During these six different optimization runs;

- "minimum" of the average coupling values of the individuals generated at each iteration

- "maximum" of the average coupling values of the individuals generated at each iteration

- "population average” of the average coupling values of the individuals generated at each iteration 
are calculated in order to monitor the progresses and performances of the these optimization runs. Graphs related to these calculations are given in Figure 6.12 through Figure 6.17.

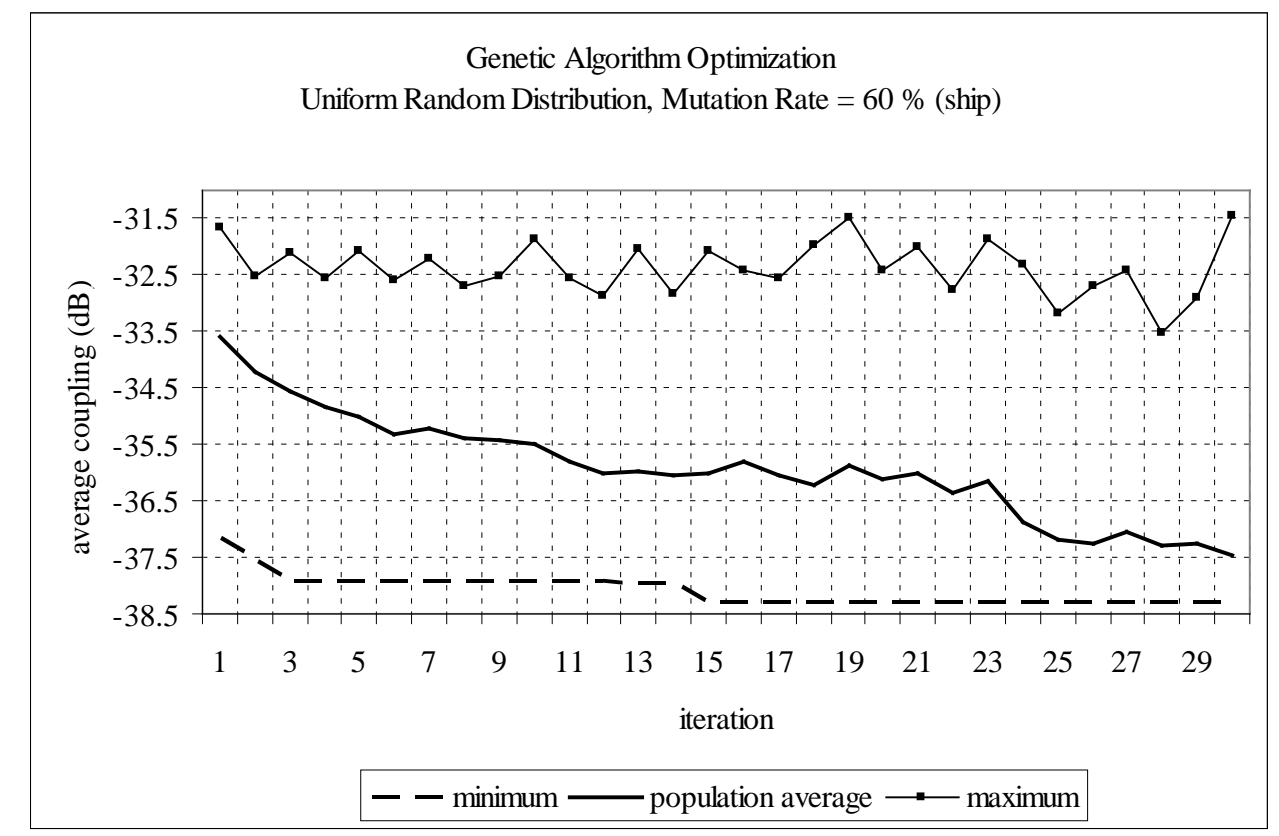

Figure 6.12 Progress of the optimization run with an initial population generated by uniform random distribution technique and with a mutation rate of $60 \%$ 


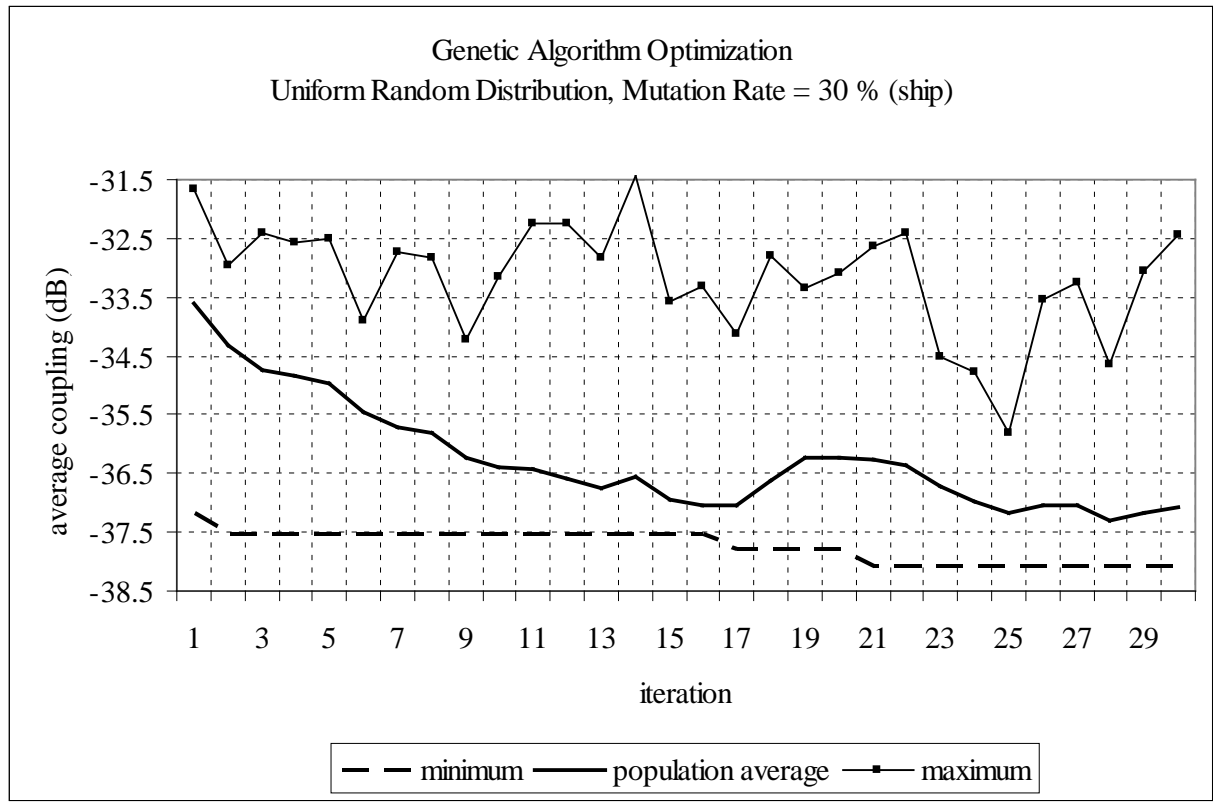

Figure 6.13 Progress of the optimization run with an initial population generated by uniform random distribution technique and with a mutation rate of $30 \%$

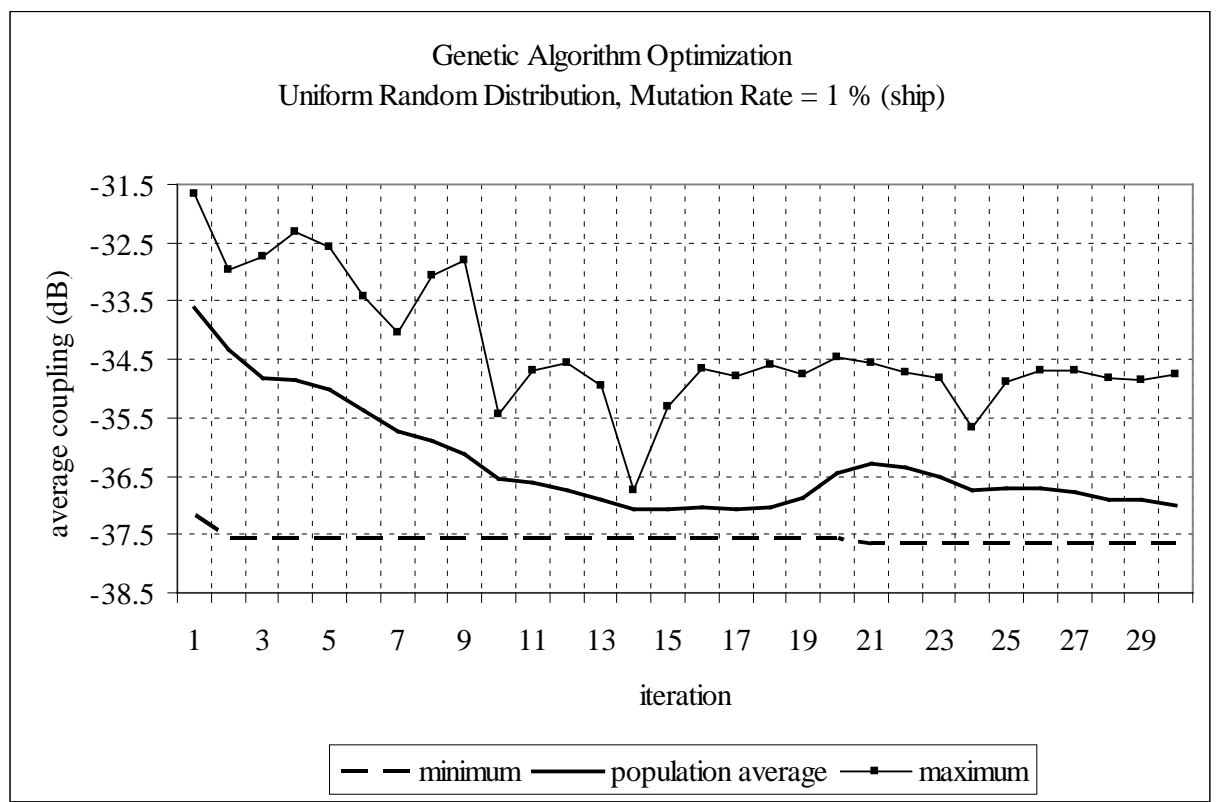

Figure 6.14 Progress of the optimization run with an initial population generated by uniform random distribution technique and with a mutation rate of $1 \%$ 


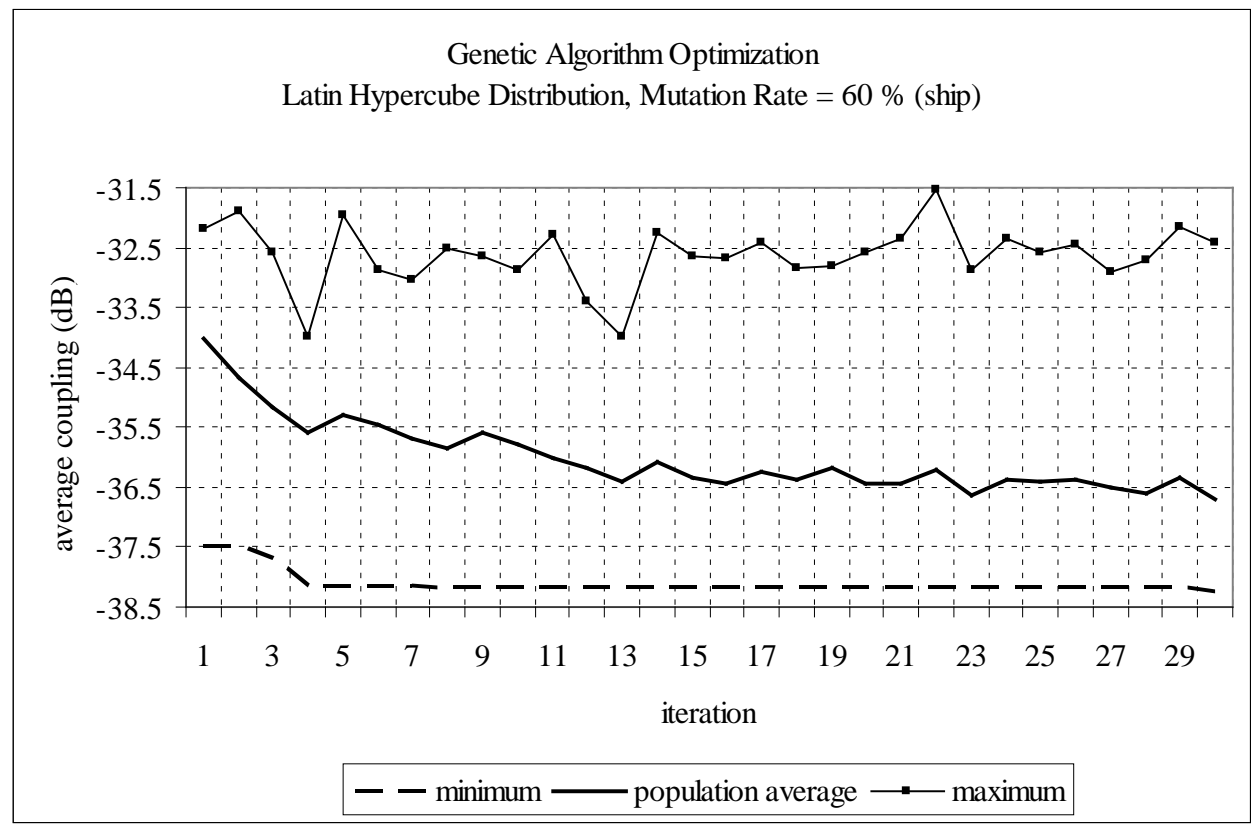

Figure 6.15 Progress of the optimization run with an initial population generated by Latin hypercube distribution technique and with a mutation rate of $60 \%$

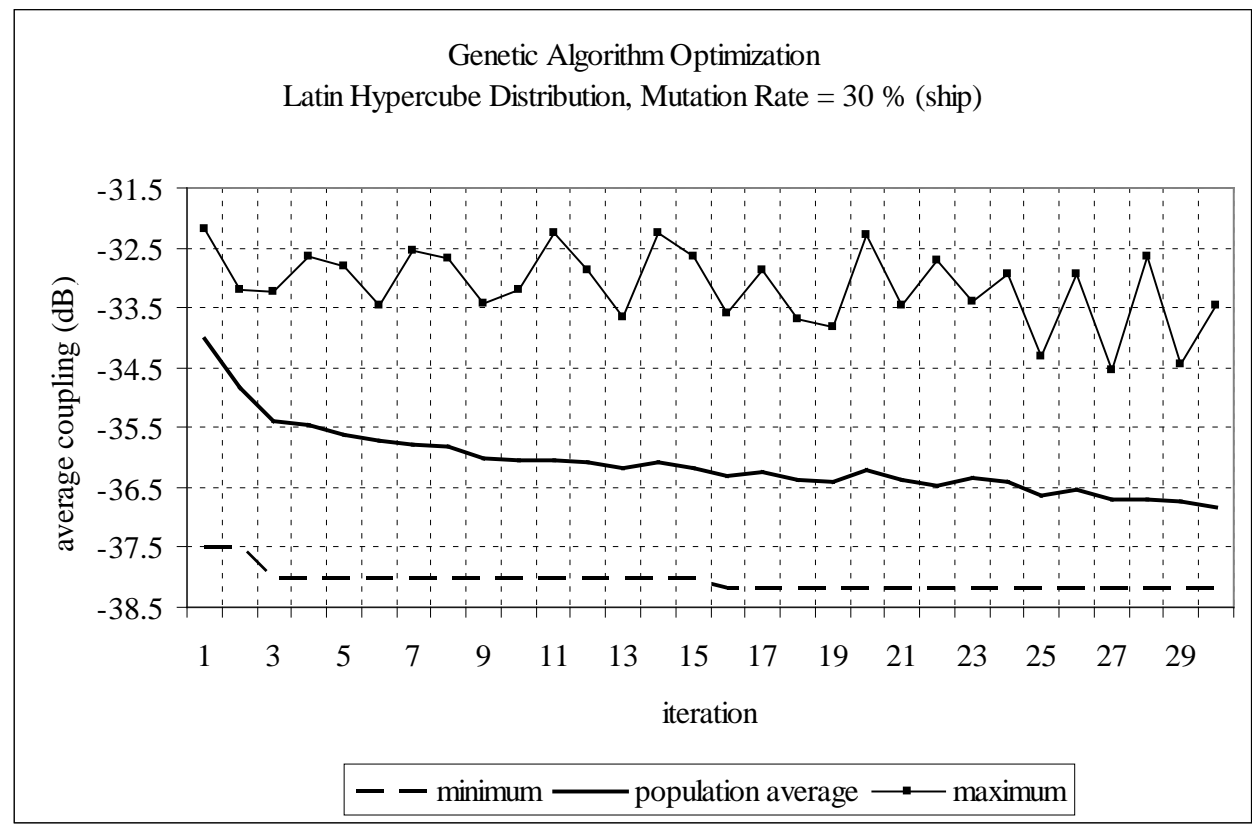

Figure 6.16 Progress of the optimization run with an initial population generated by Latin hypercube distribution technique and with a mutation rate of $30 \%$ 


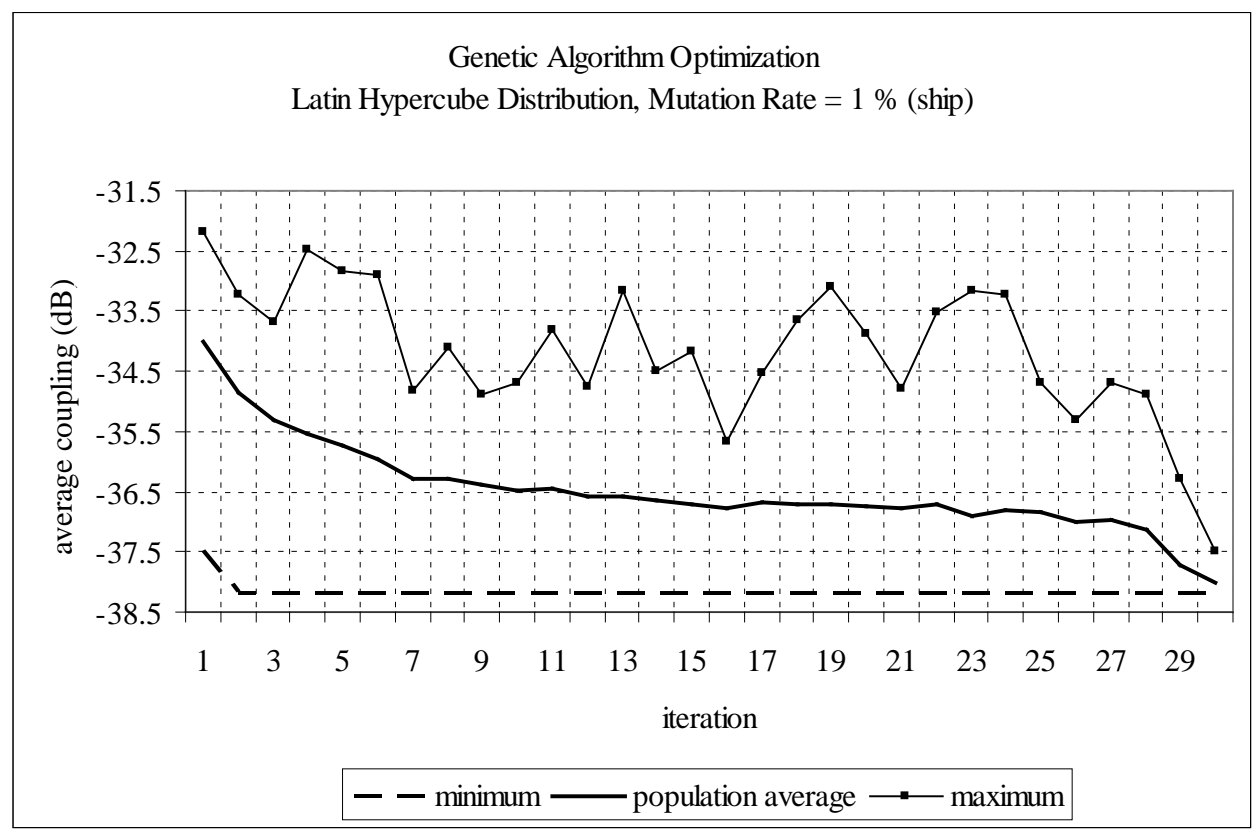

Figure 6.17 Progress of the optimization run with an initial population generated by Latin hypercube distribution technique and with a mutation rate of $1 \%$

According to Table 6.3 and Figure 6.12 through Figure 6.17:

- For all of the six antenna placements in Table 6.3, final average coupling values are very close to each other.

- Minimum (best) final average coupling between the antennas, - $38.27 \mathrm{~dB}$, is achieved in the optimization run in where the initial population is generated by uniform random distribution technique and mutation rate of $60 \%$ is used.

- Maximum (worst) final average coupling between the antennas, - 37.66 $\mathrm{dB}$, occurs in the optimization run in where the initial population is 
generated by uniform random distribution technique and mutation rate of 1 $\%$ is used.

- Coordinates of antennas in six different antenna placements also are very close to each other like the final average coupling values.

- $\quad$ The placement; where HF fore antenna is located at $x=25.9 \mathrm{~m} \& y=3.3$ $\mathrm{m}$ and HF aft antenna is located at $x=1.1 \mathrm{~m} \& y=0.5 \mathrm{~m}$, yields minimum final average coupling between the antennas. This placement is illustrated in Figure 6.18.

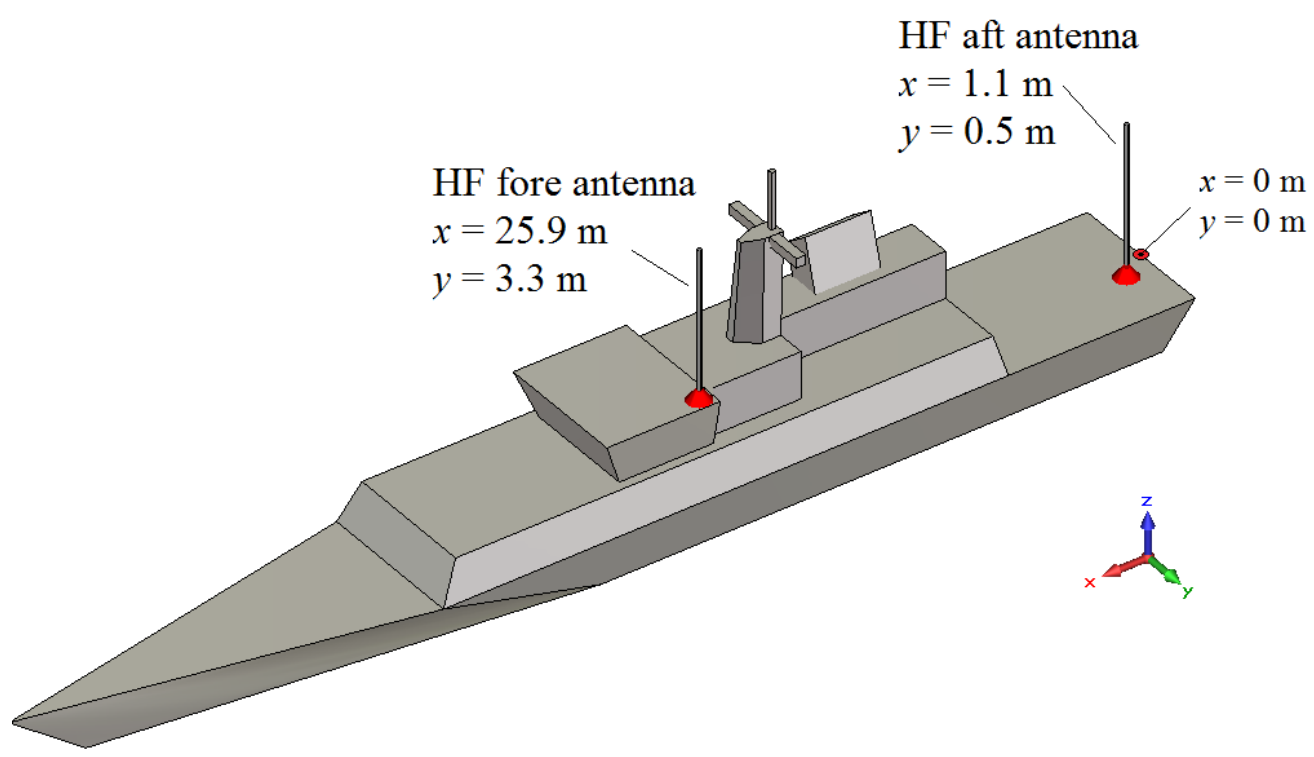

Figure 6.18 The placement yielding minimum final average coupling between HF antennas (genetic algorithm optimization)

- As it is seen in graphs above, population average of the average coupling values and minimum average coupling values decrease as the iteration number increases. In fact, in some optimization runs "minimum average 
coupling value” curve reaches its minimum value in a few iterations. Here, the more important thing is the downward trend in the population average curve. This downward trend in the population average of the average coupling values may be thought as a marker of evolution of the individuals towards the global optimum.

- It is important to note that, in the optimization runs with mutation rates of $60 \%$ and $30 \%$, the population average of the average coupling values and the maximum average coupling values fluctuate through the iterations (like in the aircraft case). Moreover, because of these fluctuations, the differences between the minimum and the maximum average coupling curves in the optimization runs with mutation rates of $60 \%$ and $30 \%$ are larger than differences in the optimization runs with mutation rate of $1 \%$. The reason for these fluctuations is the diversity in the population because of high mutation rates. The fluctuations and differences become smaller as the mutation rate decreases.

- Each of the genetic algorithm optimization runs, namely 620 simulations, last for about 26 hours (about 1 day) with a machine having 4 GB random access memory and $2.53 \mathrm{GHz}$ Intel ${ }^{\circledR}$ Core $^{\mathrm{TM}} 2$ Duo central processor unit.

\subsubsection{Optimization of Antenna Placement on Ship by Particle Swarm Optimization}

Parameters of the particle swarm optimization are set as follows in optimization of antenna placement on ship:

- Coordinates of the HF antennas on $x$ and $y$ axes are defined as variables. Therefore, variable number is defined as 4 . 
- Number of particles in the swarm is defined as 40 .

- Two different random creation functions which are based on "uniform random distribution" and "Latin hypercube distribution" are used for the generation of the initial swarm of particles.

- Maximum number of iterations is defined as 30. Algorithm stops when maximum number of iterations is reached.

- Total number of simulations is the multiplication of "the number of particles in the swarm" and "the maximum number of iterations", namely $40 \times 30=1200$.

- CST-MWS particle swarm optimizer randomly determines the weights of parameters of position and velocity vectors (explained in Section 2.2 in detail).

- Two different antenna placements are determined for each of the swarms generated by two different distribution techniques.

Coordinates of HF antennas on the ship and final average coupling values between them obtained by different optimization runs are given in Table 6.4. 
Table 6.4 Antenna placement results on ship obtained by particle swarm optimization

\begin{tabular}{|c|c|c|c|}
\hline $\begin{array}{c}\text { Distribution } \\
\text { Technique Used for } \\
\text { Generation of Initial } \\
\text { Swarm }\end{array}$ & $\begin{array}{c}\text { Coordinate } \\
\text { of HF Fore } \\
\text { Antenna }\end{array}$ & $\begin{array}{l}\text { Coordinate } \\
\text { of HF Aft } \\
\text { Antenna }\end{array}$ & $\begin{array}{l}\text { Average } \\
\text { Coupling } \\
\text { Between } \\
\text { Antennas }\end{array}$ \\
\hline $\begin{array}{l}\text { Uniform Random } \\
\text { Distribution }\end{array}$ & $\begin{array}{l}x=25.3 \mathrm{~m} \\
y=3.4 \mathrm{~m}\end{array}$ & $\begin{array}{l}x=1.3 \mathrm{~m} \\
y=0.6 \mathrm{~m}\end{array}$ & - $39.47 \mathrm{~dB}$ \\
\hline $\begin{array}{l}\text { Latin Hypercube } \\
\text { Distribution }\end{array}$ & $\begin{array}{l}x=25.1 \mathrm{~m} \\
y=3.4 \mathrm{~m}\end{array}$ & $\begin{array}{l}x=1.5 \mathrm{~m} \\
y=1.2 \mathrm{~m}\end{array}$ & - $39.58 \mathrm{~dB}$ \\
\hline
\end{tabular}

During these two different optimization runs;

- “minimum” of the average coupling values of the individuals generated at each iteration

- "maximum" of the average coupling values of the individuals generated at each iteration

- "swarm average" of the average coupling values of the individuals generated at each iteration

are calculated in order to monitor the progresses and performances of the these optimization runs. Graphs related to these calculations are given in Figure 6.19 and Figure 6.20. 


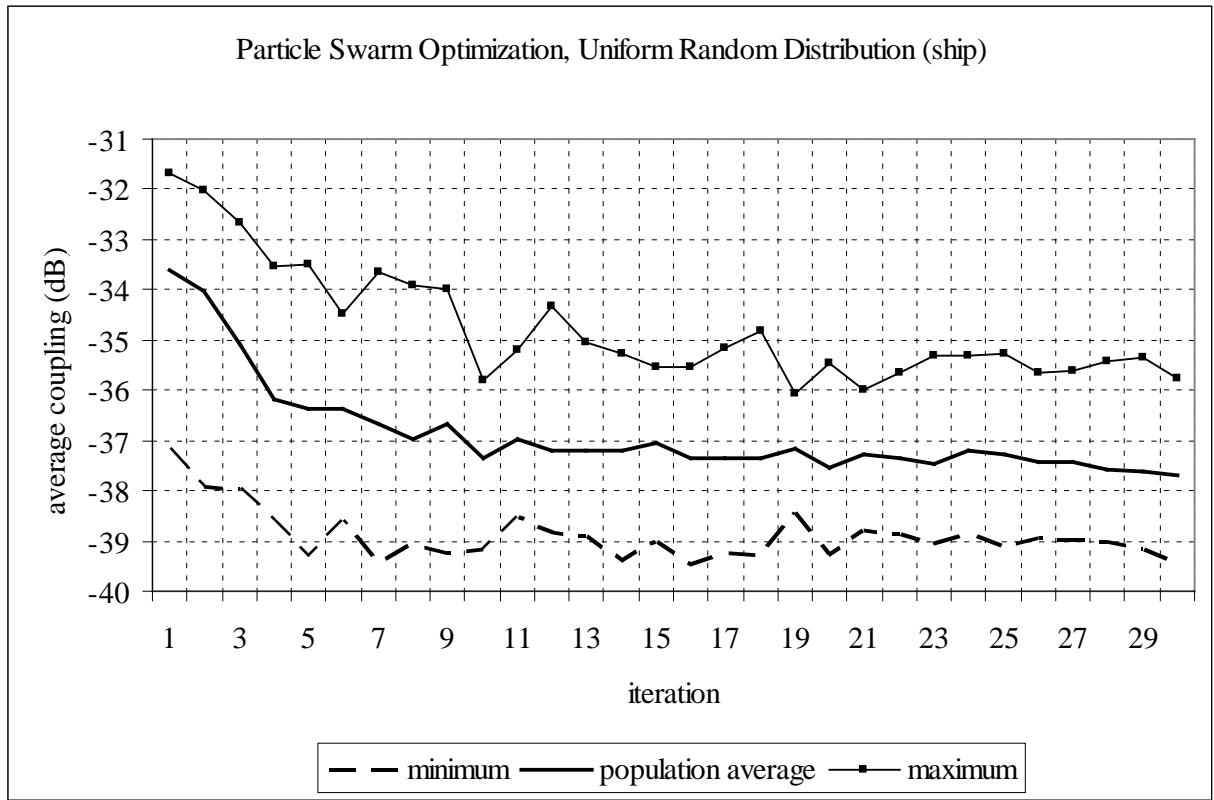

Figure 6.19 Progress of the optimization run with an initial swarm generated by uniform random distribution technique

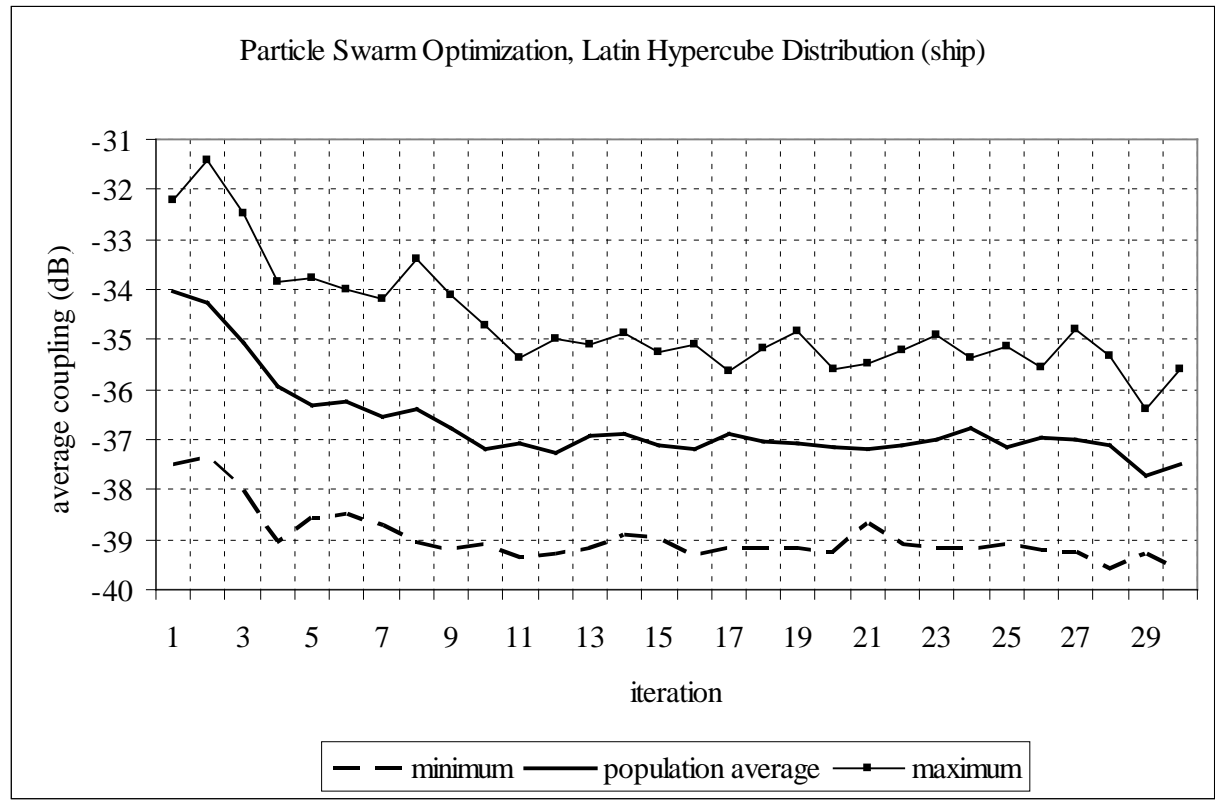

Figure 6.20 Progress of the optimization run with an initial swarm generated by Latin hypercube distribution technique 
According to Table 6.4, Figure 6.19 and Figure 6.20:

- For both of the antenna placements in Table 6.4, final average coupling values are very close to each other.

- Minimum (best) final average coupling between the antennas, - $39.58 \mathrm{~dB}$, is achieved in the optimization run in where the initial swarm is generated by Latin hypercube distribution technique.

- Coordinates of antennas in two different antenna placements also are very close to each other like the final average coupling values.

- The placement; where HF fore antenna is located at $x=25.1 \mathrm{~m} \& y=3.4$ $\mathrm{m}$ and HF aft antenna is located at $x=1.5 \mathrm{~m} \& y=1.2 \mathrm{~m}$, yields minimum final average coupling between the antennas. This placement is illustrated in Figure 6.21.

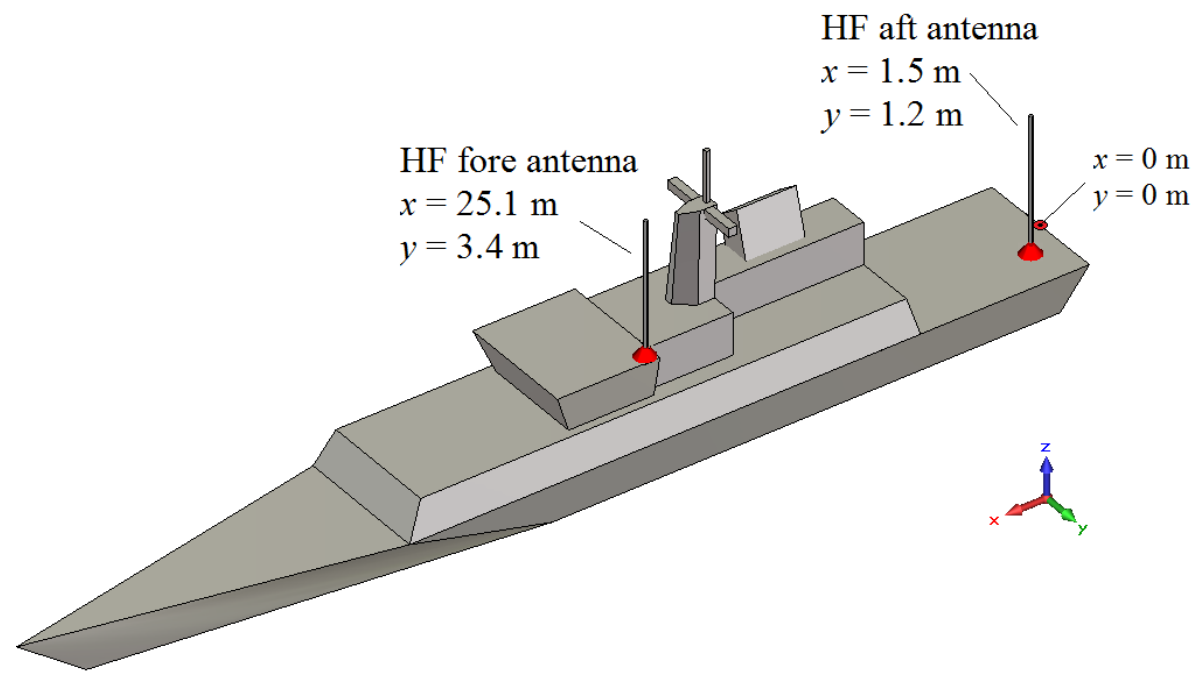

Figure 6.21 The placement yielding minimum final average coupling between HF antennas (particle swarm optimization) 
- As it is seen in Figure 6.19 and Figure 6.20, swarm average of the average coupling values and minimum average coupling values decrease as the iteration number increases. This downward trend in the swarm average of the average coupling values may be thought as a marker of tendency of the whole swarm toward the global optimum.

- The fluctuations in the swarm average of the average coupling values and the maximum average coupling values are very small in contrast to the fluctuations in the genetic algorithm optimization runs with mutation rates of $60 \%$ and $30 \%$. Moreover, the difference between the minimum and the maximum average coupling values are smaller when compared with the differences in the genetic algorithm optimization runs with mutation rates of $60 \%$ and $30 \%$. Since there is no mutation operator in particle swarm optimization, these fluctuations and the difference between the curves resemble the fluctuations and the differences in the genetic algorithm optimization runs with mutation rate of $1 \%$.

- Each of the particle swarm optimization runs, namely 1200 simulations, last for about 50 hours (about 2 days) with a machine having 4 GB random access memory and $2.53 \mathrm{GHz}$ Intel ${ }^{\circledR}$ Core $^{\mathrm{TM}} 2$ Duo central processor unit. 


\title{
CHAPTER 7
}

\section{FAR-FIELD RADIATION PATTERN PERFORMANCES OF ANTENNAS}

\begin{abstract}
After the optimal placements of aircraft and ship antennas are determined, farfield radiation pattern performances of those antennas for their optimal placements are analyzed in terms of directivity and coverage at the end of this thesis work.
\end{abstract}

Far-field analyses deal with the field behavior far away from the antennas. Throughout the far-field analyses, far-field radiation patterns of the aircraft and ship antennas are calculated at distinct frequencies such that whole frequency bands of operation of the antennas are sampled uniformly. These far-field radiation patterns are represented graphically as plots of "directivity" in the spherical coordinate system. From these directivity plots, coverage on each of the aircraft and ship platforms is figured out.

The directivity of an antenna is an indicator of its radiation and reception performance. It is defined as the ratio of the radiation intensity in a given direction from the antenna to the radiation intensity averaged over all directions and represented mathematically in Equation 7.1 as a function standard spherical coordinates. Directivity of an isotropic antenna is $0 \mathrm{~dB}$ since it radiates equally to all directions (for all azimuth “ $\phi$ ” and elevation “ $\theta$ ” angles). Generally, the directivities of all other antennas are expressed with respect to directivity of an isotropic antenna in terms of $\mathrm{dBi}$. 
$\operatorname{Directivity}(\theta, \phi)=\frac{(\text { radiated power density }(\theta, \phi))}{(\text { total radiated power } / 4 \pi)}$

where $\theta$ and $\phi$ are standard spherical coordinate angles and $4 \pi$ is the total solid angle for a sphere.

In order to analyze the far-field radiation performances of aircraft and ship antennas; at each distinct frequency, directivities of them are compared with the directivities of monopole antennas on an infinite perfectly conducting ground plane, having the same electrical and physical properties with those antennas.

\subsection{Far-field Radiation Pattern Performances of Aircraft Antennas}

The optimal antenna placement yielding minimum average coupling between the aircraft antennas is determined by Genetic Algorithm Optimization. In this optimization, the initial population is generated by Latin hypercube distribution technique and mutation rate of $60 \%$ is used. In the optimal placement, VHF upper antenna is located at $x=0.86 \mathrm{~m}$ and VHF lower antenna is located at $x=7.88 \mathrm{~m}$.

In this antenna placement, VHF upper and lower antennas radiate/receive in the upper and lower hemispheres respectively. In order to determine the radiation performances of these antennas on the upper and lower hemispheres, directivities of them are compared with the directivities of a monopole antenna on an infinite ground plane as follows:

- Directivities of the aircraft antennas are calculated at $30 \mathrm{MHz}, 40 \mathrm{MHz}, 50$ $\mathrm{MHz}, 60 \mathrm{MHz}, 70 \mathrm{MHz}$ and $80 \mathrm{MHz}$. 
- Directivities of a VHF monopole antenna on an "infinite ground plane”, having the same electrical and physical properties with aircraft antennas are calculated at same distinct frequencies.

- At each frequency, "the ranges of azimuth and elevation angles ( $\phi$ and $\theta$ )” in where the directivity of the monopole antenna on an infinite ground plane is greater than $0 \mathrm{dBi}$, is determined.

- At each frequency, directivity of each aircraft antenna is examined within the angular ranges mentioned in the bullet above. If minimum of $50 \%$ of directivity values of the aircraft antenna within these ranges is greater than $0 \mathrm{dBi}$, then that antenna is assumed to have "sufficient coverage", which means, that antenna radiates/receives sufficiently.

\subsubsection{Far-field Radiation Pattern Analyses for Aircraft Antennas at $\mathbf{3 0}$ MHz}

Three-dimensional and two-dimensional far-field radiation patterns of a VHF monopole antenna on an infinite ground plane at $30 \mathrm{MHz}$ are given in Figure 7.1. According to this figure, for $34^{\circ} \leq \theta \leq 90^{\circ}$ (within a range of $56^{\circ}$ ) and $0^{\circ} \leq \phi \leq 360^{\circ}$, directivity of monopole antenna on an infinite ground plane is greater than $0 \mathrm{dBi}$. 
3D-directivity values of monopole antenna on an infinite ground plane $(30 \mathrm{MHz})$

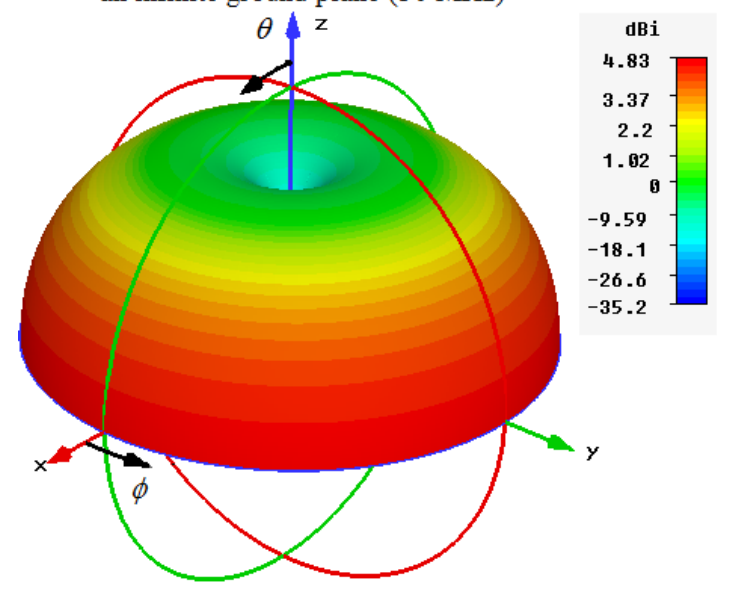

2D-directivity values of monopole antenna on an infinite ground plane $(30 \mathrm{MHz})$

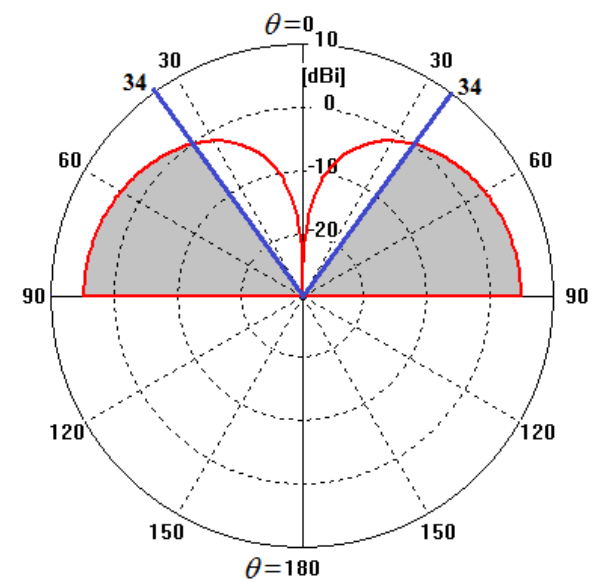

Figure 7.1 Far-field radiation patterns of monopole antenna at $30 \mathrm{MHz}$

Figure 7.2 shows the far-field radiation pattern of VHF upper antenna which is spatially filtered and examined for $34^{\circ} \leq \theta \leq 90^{\circ}$ and $0^{\circ} \leq \phi \leq 360^{\circ}$. Figure 7.3 shows the far-field radiation pattern of VHF lower antenna which is spatially filtered and examined for $90^{\circ} \leq \theta \leq 146^{\circ}$ and $0^{\circ} \leq \phi \leq 360^{\circ}$. According to these figures:

- $66 \%$ (greater than $50 \%$ ) of the directivity values of VHF upper antenna for $34^{\circ} \leq \theta \leq 90^{\circ}$ and $0^{\circ} \leq \phi \leq 360^{\circ}$ is greater than $0 \mathrm{dBi}$.

- $82 \%$ (greater than $50 \%$ ) of the directivity values of VHF lower antenna for $90^{\circ} \leq \theta \leq 146^{\circ}$ and $0^{\circ} \leq \phi \leq 360^{\circ}$ is greater than $0 \mathrm{dBi}$.

As a result both antennas are considered to have "sufficient coverage" at $30 \mathrm{MHz}$ at their optimal places where the average coupling between them is minimum. 


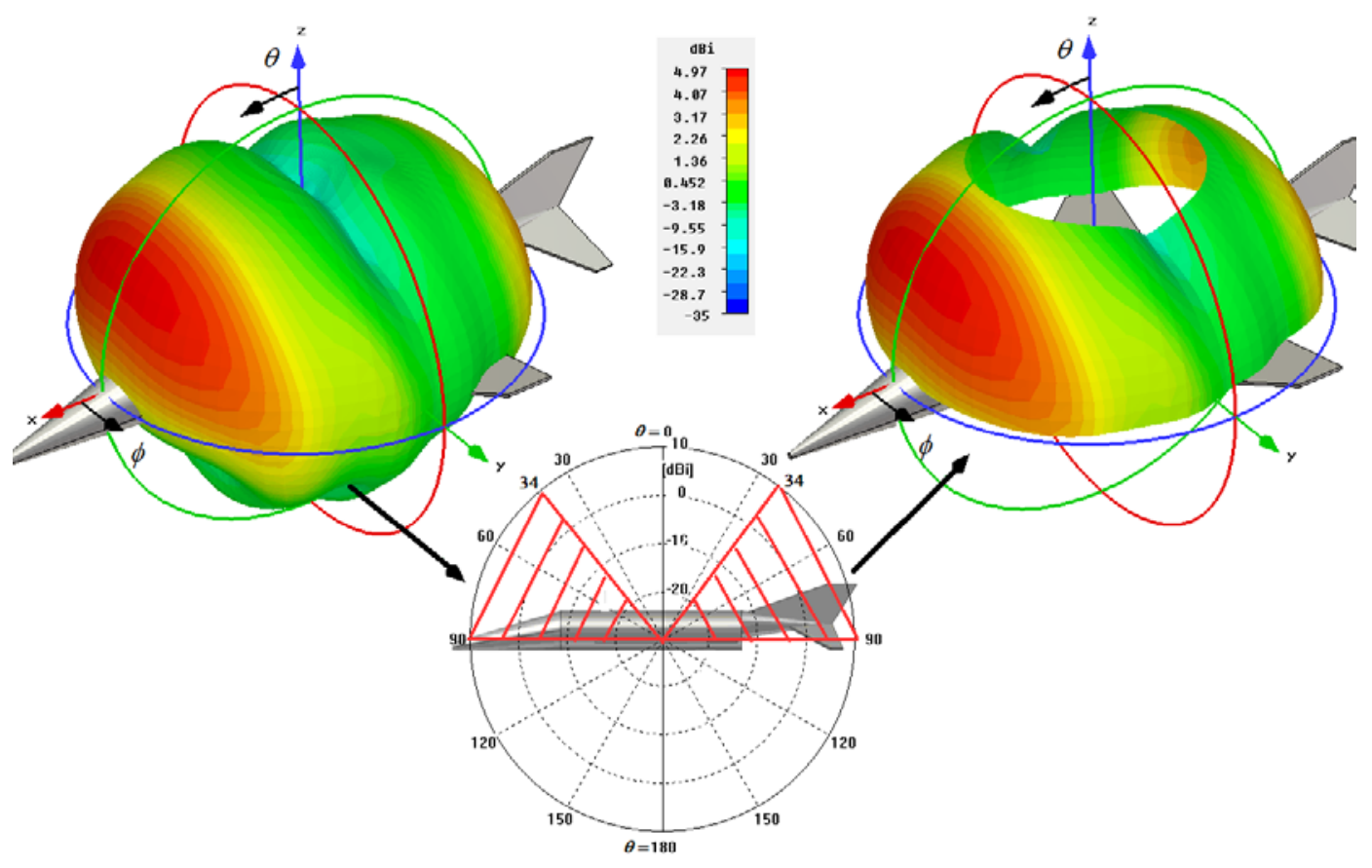

Figure 7.2 Far-field radiation pattern of VHF upper antenna at $30 \mathrm{MHz}$

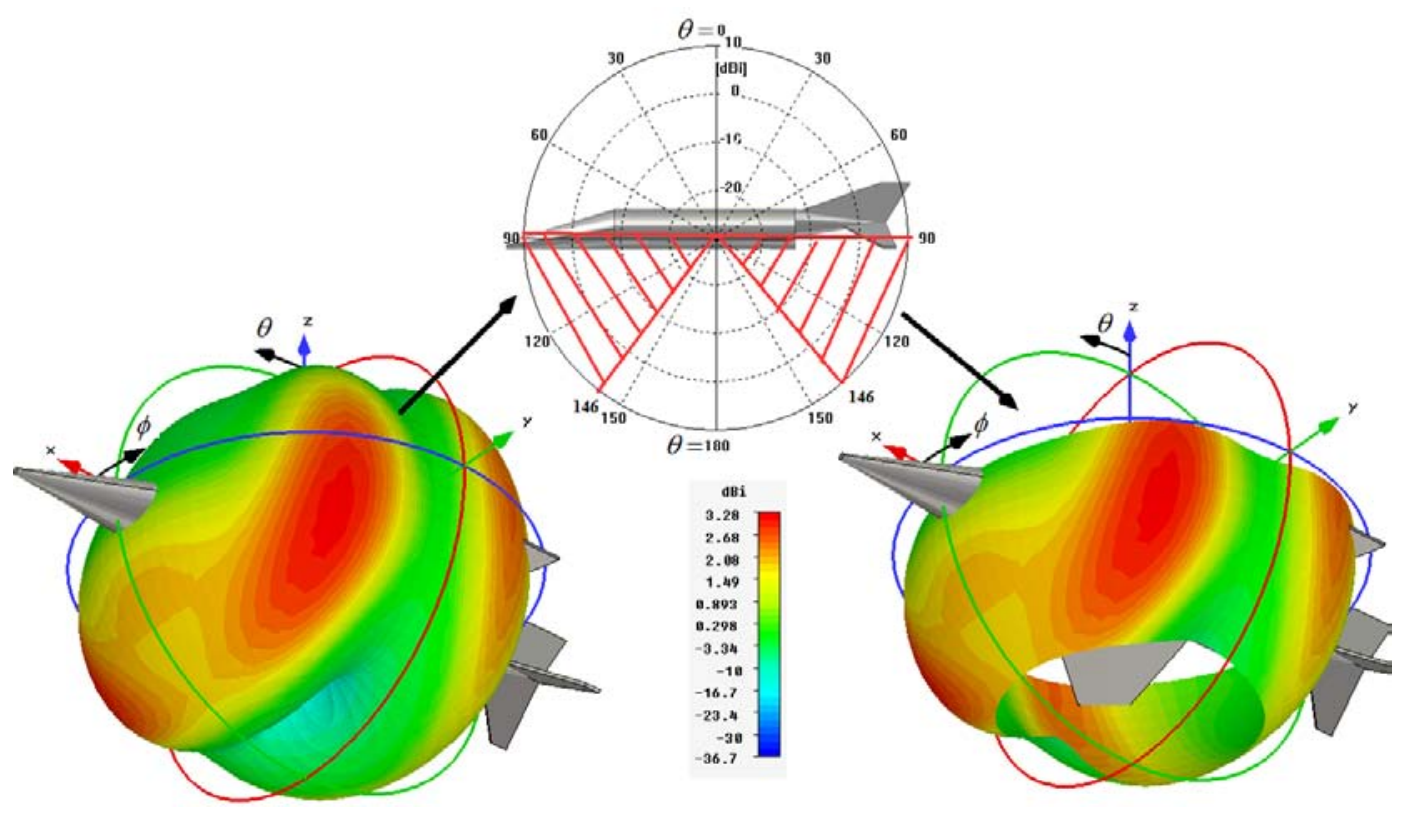

Figure 7.3 Far-field radiation pattern of VHF lower antenna at $30 \mathrm{MHz}$ 


\subsubsection{Far-field Radiation Pattern Analyses for Aircraft Antennas at $\mathbf{4 0}$ MHz}

Three-dimensional and two-dimensional far-field radiation patterns of a VHF monopole antenna on an infinite ground plane at $40 \mathrm{MHz}$ are given in Figure 7.4. According to this figure, for $37^{\circ} \leq \theta \leq 90^{\circ}$ (within a range of $53^{\circ}$ ) and $0^{\circ} \leq \phi \leq 360^{\circ}$, directivity of monopole antenna on an infinite ground plane is greater than $0 \mathrm{dBi}$.
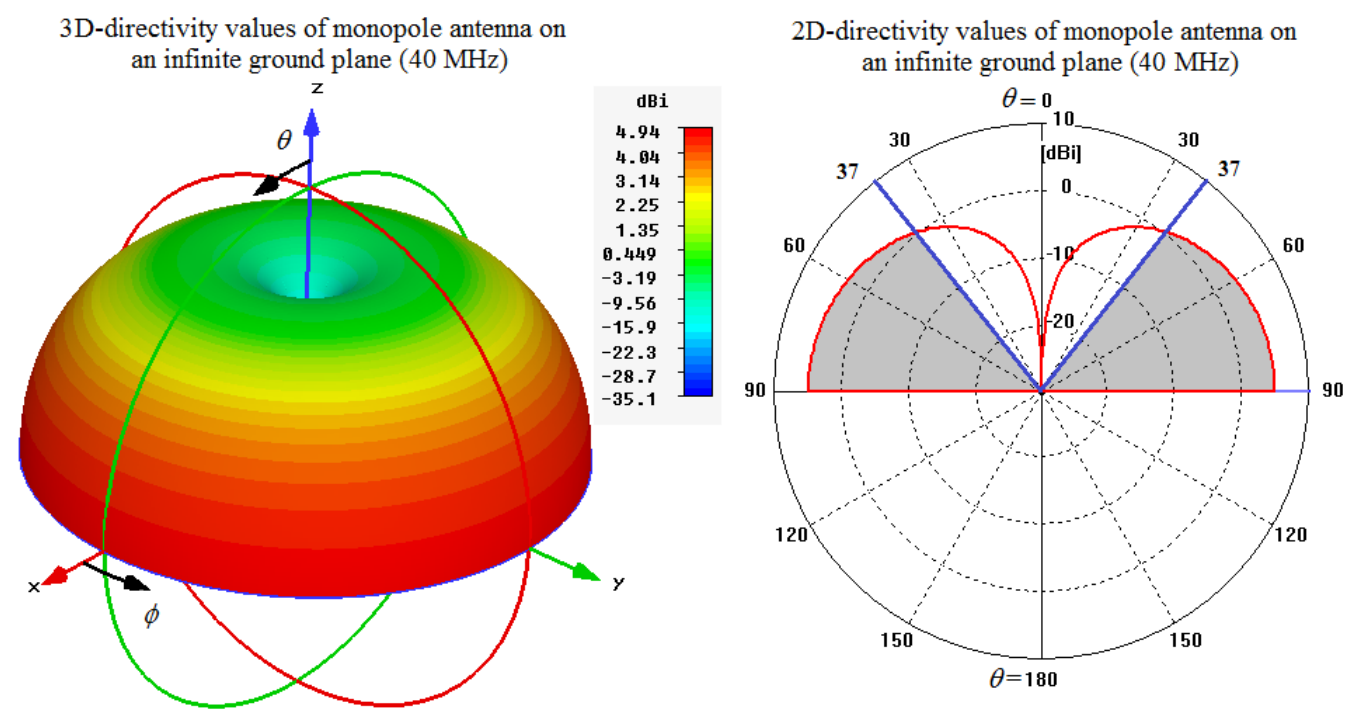

Figure 7.4 Far-field radiation patterns of monopole antenna at $40 \mathrm{MHz}$

Figure 7.5 shows the far-field radiation pattern of VHF upper antenna which is spatially filtered and examined for $37^{\circ} \leq \theta \leq 90^{\circ}$ and $0^{\circ} \leq \phi \leq 360^{\circ}$ and Figure 7.6 shows the far-field radiation pattern of VHF lower antenna which is spatially filtered and examined for $90^{\circ} \leq \theta \leq 143^{\circ}$ and $0^{\circ} \leq \phi \leq 360^{\circ}$. According to these figures: 
- $72 \%$ (greater than $50 \%$ ) of the directivity values of VHF upper antenna for $37^{\circ} \leq \theta \leq 90^{\circ}$ and $0^{\circ} \leq \phi \leq 360^{\circ}$ is greater than $0 \mathrm{dBi}$.

- $79 \%$ (greater than $50 \%$ ) of the directivity values of VHF lower antenna for $90^{\circ} \leq \theta \leq 143^{\circ}$ and $0^{\circ} \leq \phi \leq 360^{\circ}$ is greater than $0 \mathrm{dBi}$.

As a result both antennas are considered to have "sufficient coverage" at $40 \mathrm{MHz}$ at their optimal places where the average coupling between them is minimum.

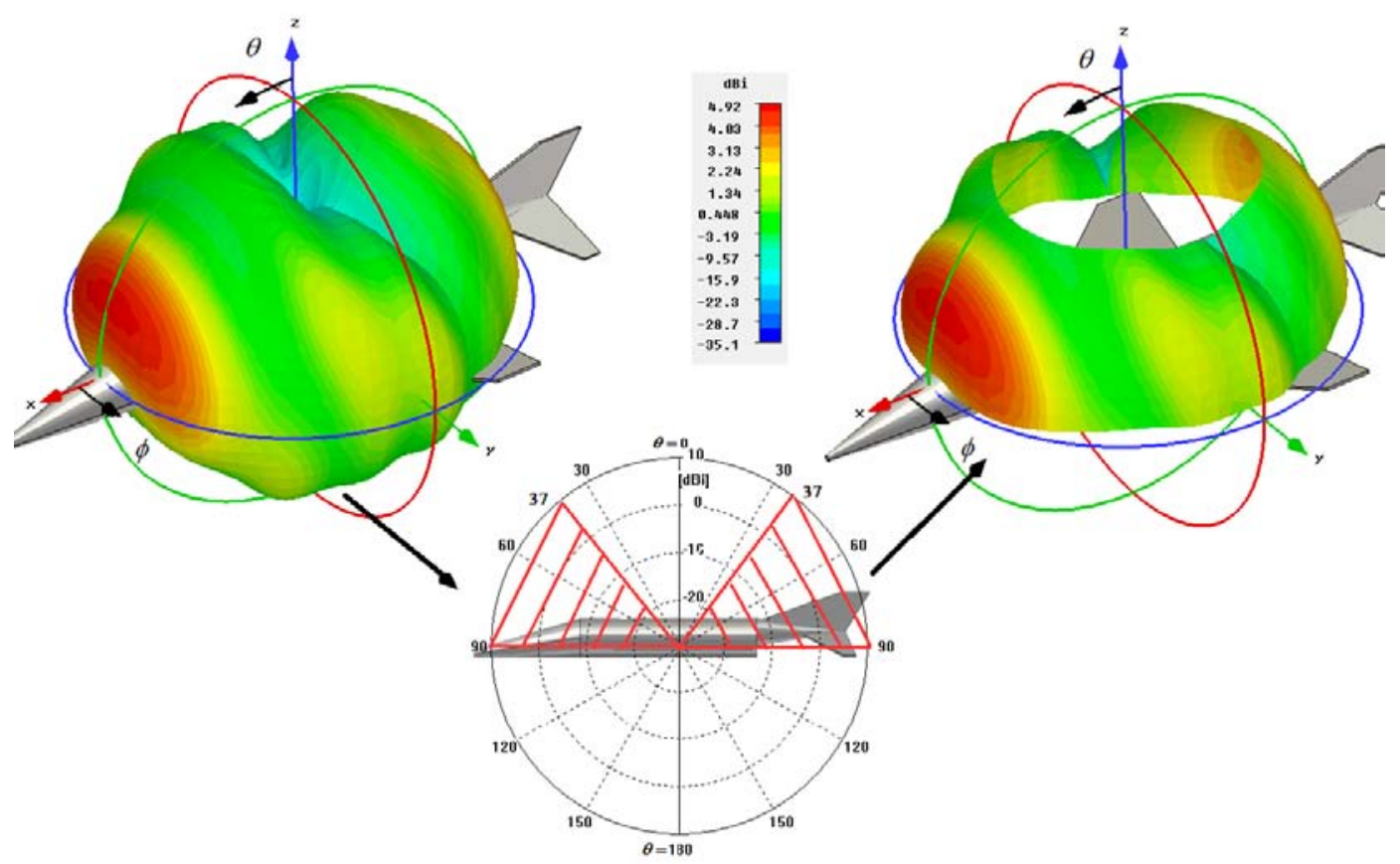

Figure 7.5 Far-field radiation pattern of VHF upper antenna at $40 \mathrm{MHz}$ 


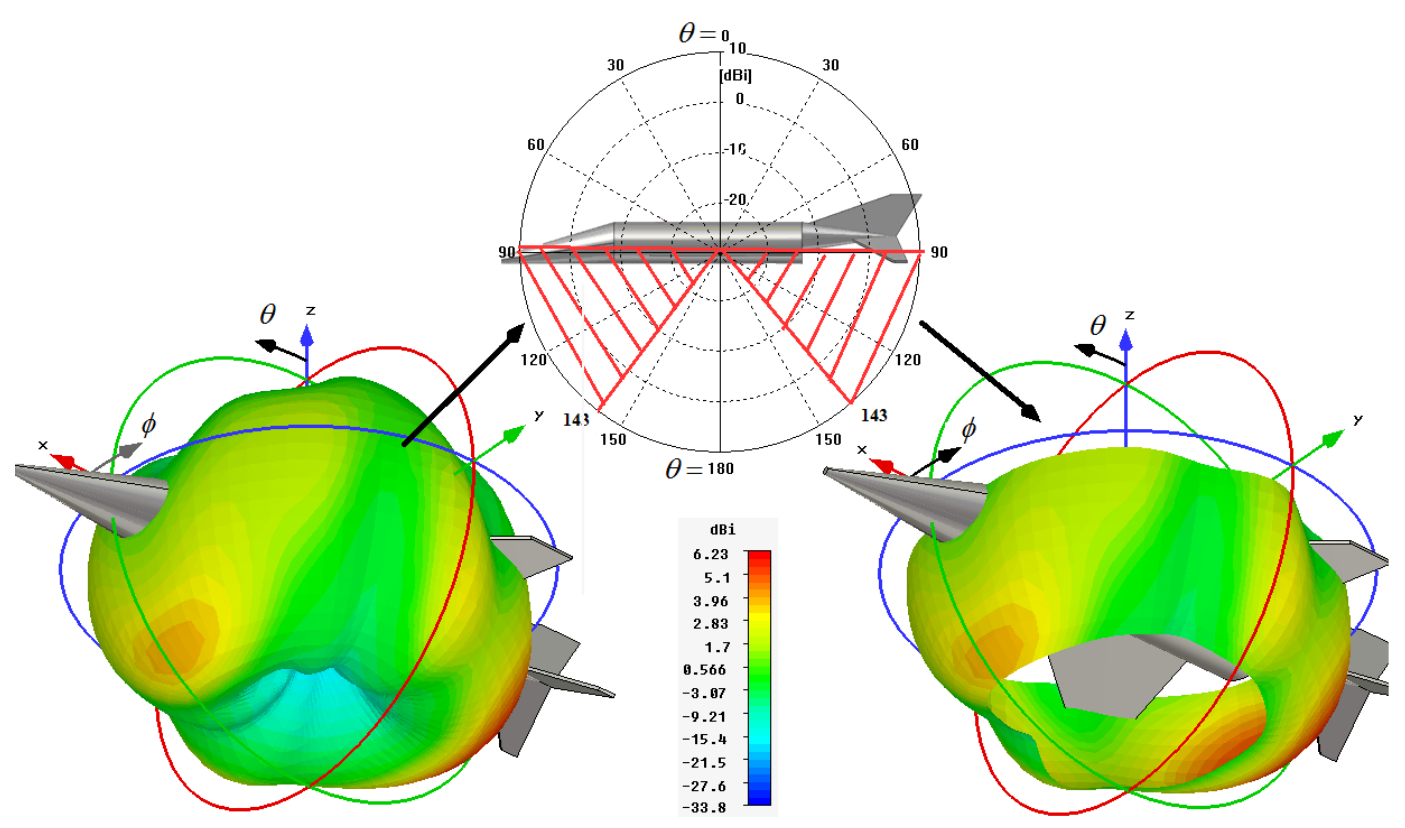

Figure 7.6 Far-field radiation pattern of VHF lower antenna at $40 \mathrm{MHz}$

\subsubsection{Far-field Radiation Pattern Analyses for Aircraft Antennas at 50 MHz}

Three-dimensional and two-dimensional far-field radiation patterns of a VHF monopole antenna on an infinite ground plane at $50 \mathrm{MHz}$ are given in Figure 7.7. According to this figure, for $37^{\circ} \leq \theta \leq 90^{\circ}$ (within a range of $53^{\circ}$ ) and $0^{\circ} \leq \phi \leq 360^{\circ}$, directivity of monopole antenna on an infinite ground plane is greater than $0 \mathrm{dBi}$. 


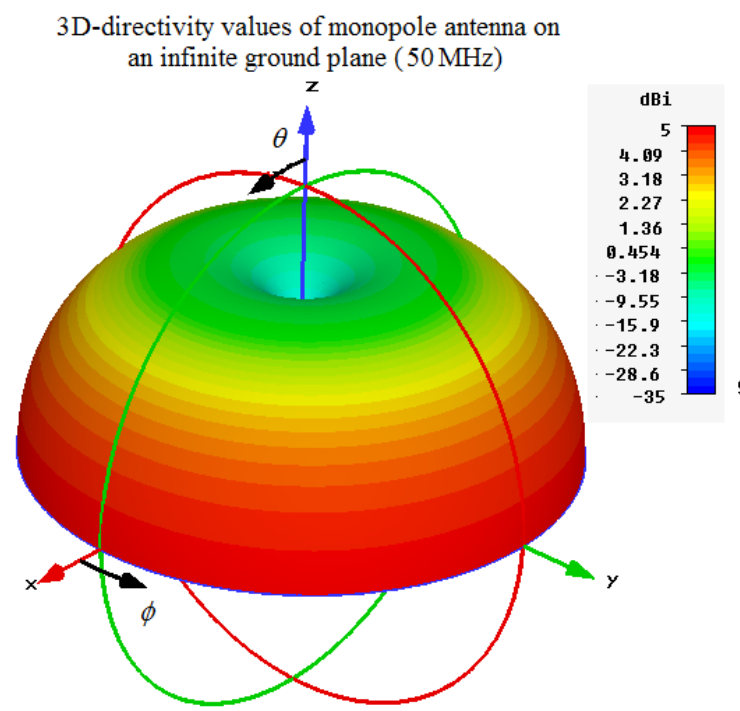

2D-directivity values of monopole antenna on an infinite ground plane $(50 \mathrm{MHz})$

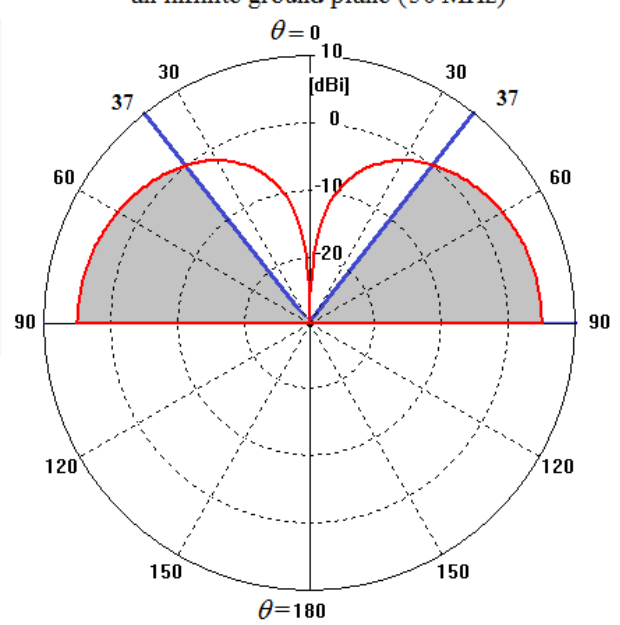

Figure 7.7 Far-field radiation patterns of monopole antenna at $50 \mathrm{MHz}$

Figure 7.8 shows the far-field radiation pattern of VHF upper antenna which is spatially filtered and examined for $37^{\circ} \leq \theta \leq 90^{\circ}$ and $0^{\circ} \leq \phi \leq 360^{\circ}$ and Figure 7.9 shows the far-field radiation pattern of VHF lower antenna which is spatially filtered and examined for $90^{\circ} \leq \theta \leq 143^{\circ}$ and $0^{\circ} \leq \phi \leq 360^{\circ}$. According to these figures:

- $72 \%$ (greater than $50 \%$ ) of the directivity values of VHF upper antenna for $37^{\circ} \leq \theta \leq 90^{\circ}$ and $0^{\circ} \leq \phi \leq 360^{\circ}$ is greater than $0 \mathrm{dBi}$.

- $80 \%$ (greater than $50 \%$ ) of the directivity values of VHF lower antenna for $90^{\circ} \leq \theta \leq 143^{\circ}$ and $0^{\circ} \leq \phi \leq 360^{\circ}$ is greater than $0 \mathrm{dBi}$.

As a result both antennas are considered to have "sufficient coverage" at $50 \mathrm{MHz}$ at their optimal places where the average coupling between them is minimum. 


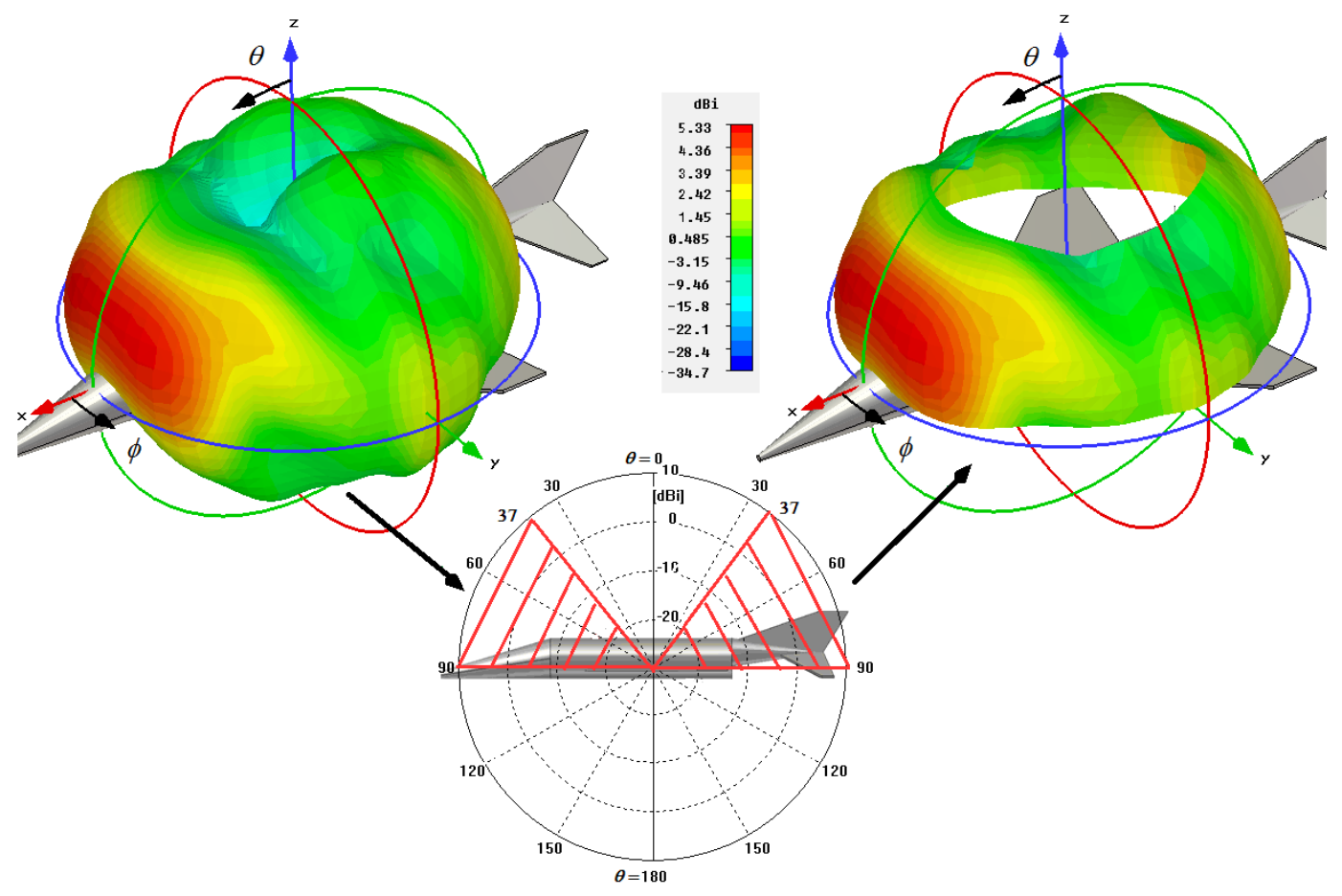

Figure 7.8 Far-field radiation pattern of VHF upper antenna at $50 \mathrm{MHz}$

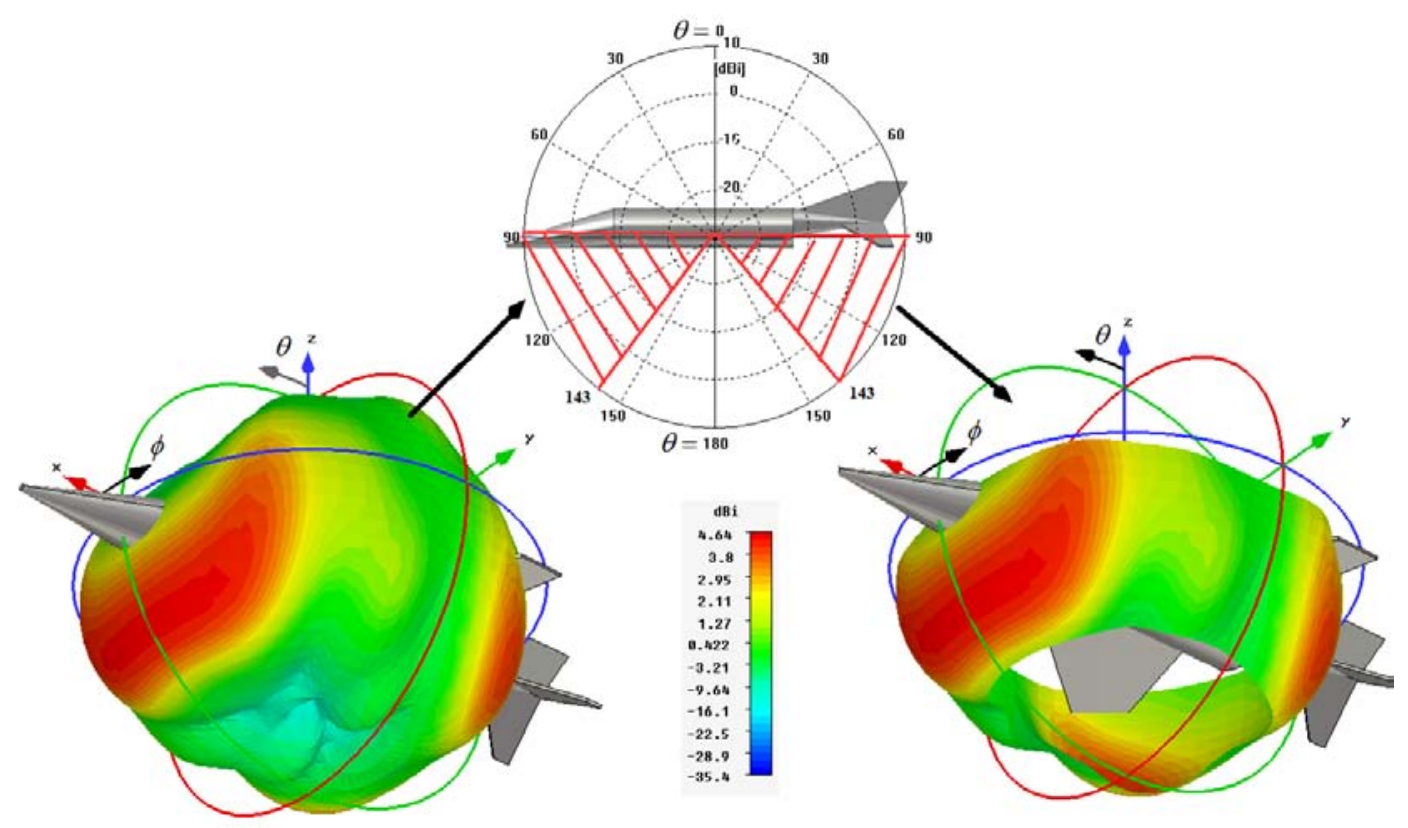

Figure 7.9 Far-field radiation pattern of VHF lower antenna at $50 \mathrm{MHz}$ 


\subsubsection{Far-field Radiation Pattern Analyses for Aircraft Antennas at 60 MHz}

Three-dimensional and two-dimensional far-field radiation patterns of a VHF monopole antenna on an infinite ground plane at $60 \mathrm{MHz}$ are given in Figure 7.10. According to this figure, for $39^{\circ} \leq \theta \leq 90^{\circ}$ (within a range of $51^{\circ}$ ) and $0^{\circ} \leq \phi \leq 360^{\circ}$, directivity of monopole antenna on an infinite ground plane is greater than $0 \mathrm{dBi}$.
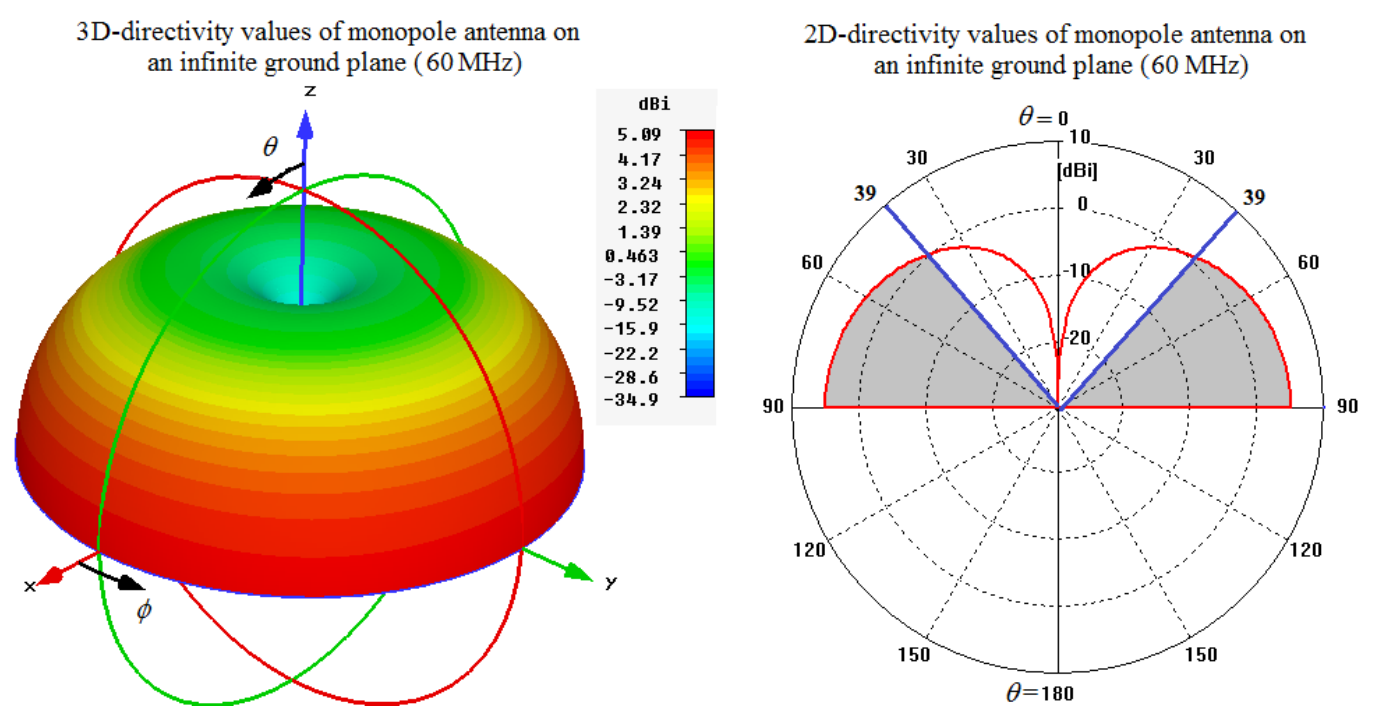

Figure 7.10 Far-field radiation patterns of monopole antenna at $60 \mathrm{MHz}$

Figure 7.11 shows the far-field radiation pattern of VHF upper antenna which is spatially filtered and examined for $39^{\circ} \leq \theta \leq 90^{\circ}$ and $0^{\circ} \leq \phi \leq 360^{\circ}$ and Figure 7.12 shows the far-field radiation pattern of VHF lower antenna which is spatially filtered and examined for $90^{\circ} \leq \theta \leq 141^{\circ}$ and $0^{\circ} \leq \phi \leq 360^{\circ}$. According to these figures: 
- $78 \%$ (greater than $50 \%$ ) of the directivity values of VHF upper antenna for $39^{\circ} \leq \theta \leq 90^{\circ}$ and $0^{\circ} \leq \phi \leq 360^{\circ}$ is greater than $0 \mathrm{dBi}$.

- $68 \%$ (greater than $50 \%$ ) of the directivity values of VHF lower antenna for $90^{\circ} \leq \theta \leq 141^{\circ}$ and $0^{\circ} \leq \phi \leq 360^{\circ}$ is greater than $0 \mathrm{dBi}$.

As a result both antennas are considered to have "sufficient coverage" at $60 \mathrm{MHz}$ at their optimal places where the average coupling between them is minimum.

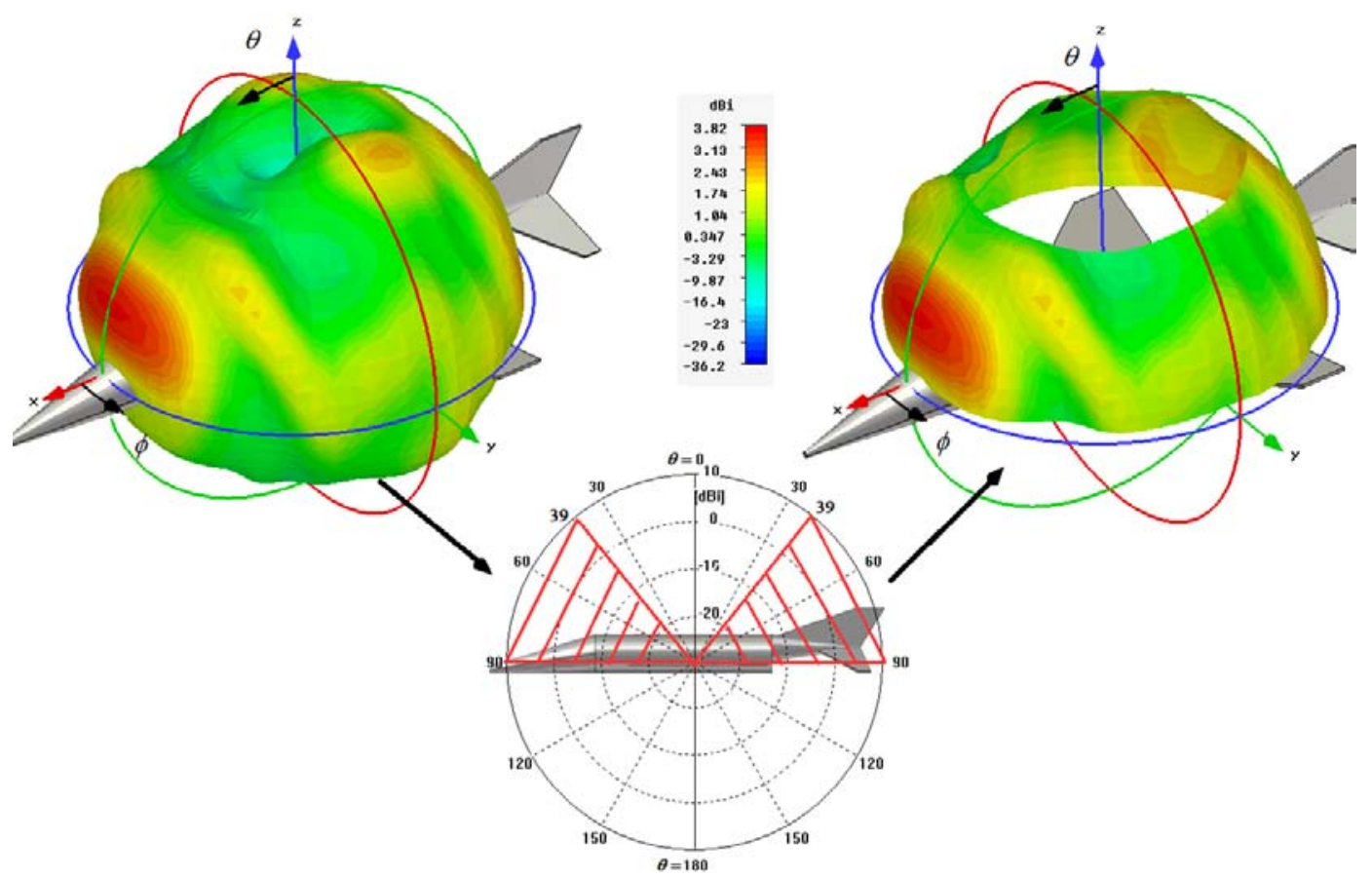

Figure 7.11 Far-field radiation pattern of VHF upper antenna at $60 \mathrm{MHz}$ 


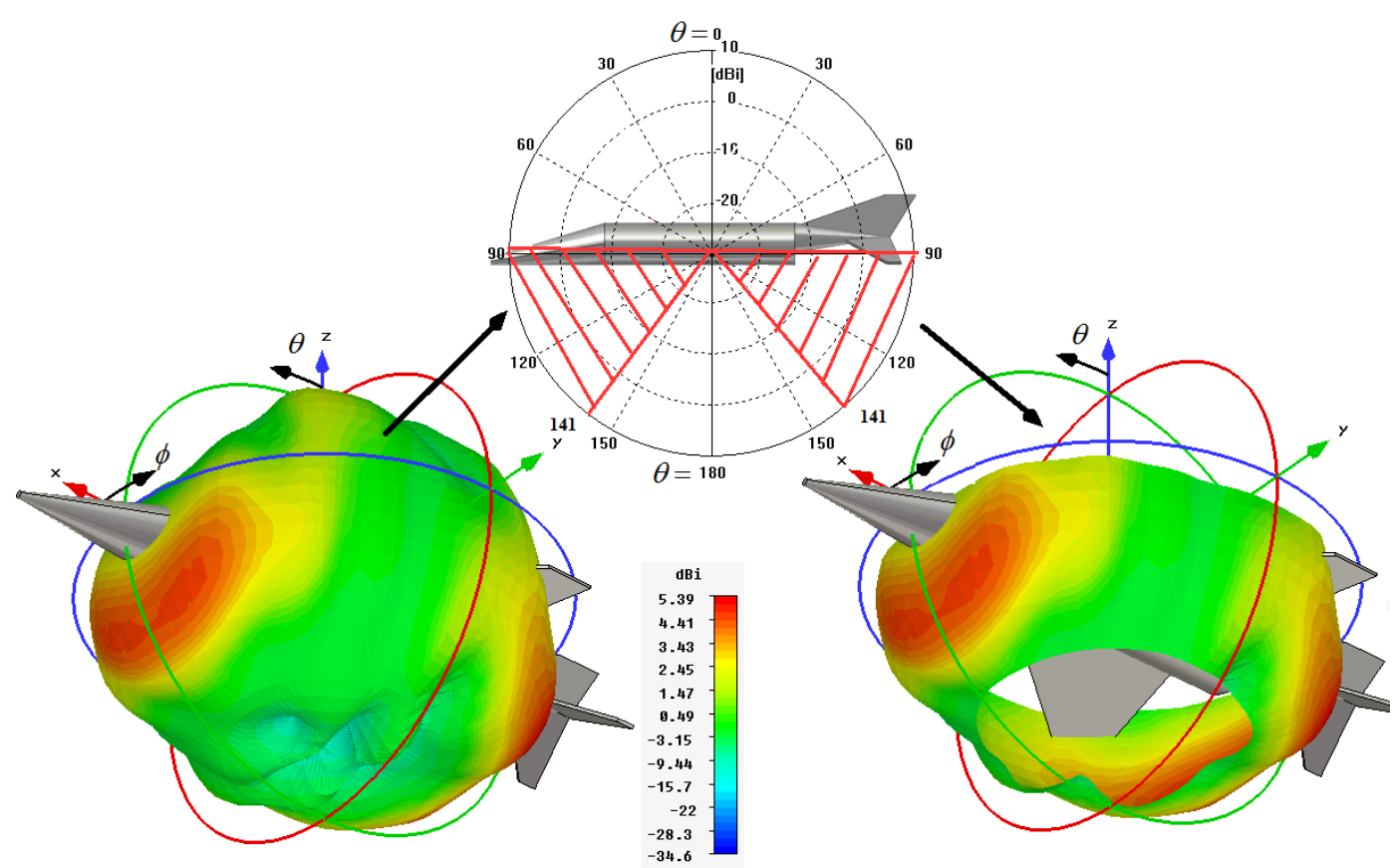

Figure 7.12 Far-field radiation pattern of VHF lower antenna at $60 \mathrm{MHz}$

\subsubsection{Far-field Radiation Pattern Analyses for Aircraft Antennas at $\mathbf{7 0}$ MHz}

Three-dimensional and two-dimensional far-field radiation patterns of a VHF monopole antenna on an infinite ground plane at $70 \mathrm{MHz}$ are given in Figure 7.13. According to this figure, for $40^{\circ} \leq \theta \leq 90^{\circ}$ (within a range of $50^{\circ}$ ) and $0^{\circ} \leq \phi \leq 360^{\circ}$, directivity of monopole antenna on an infinite ground plane is greater than $0 \mathrm{dBi}$. 

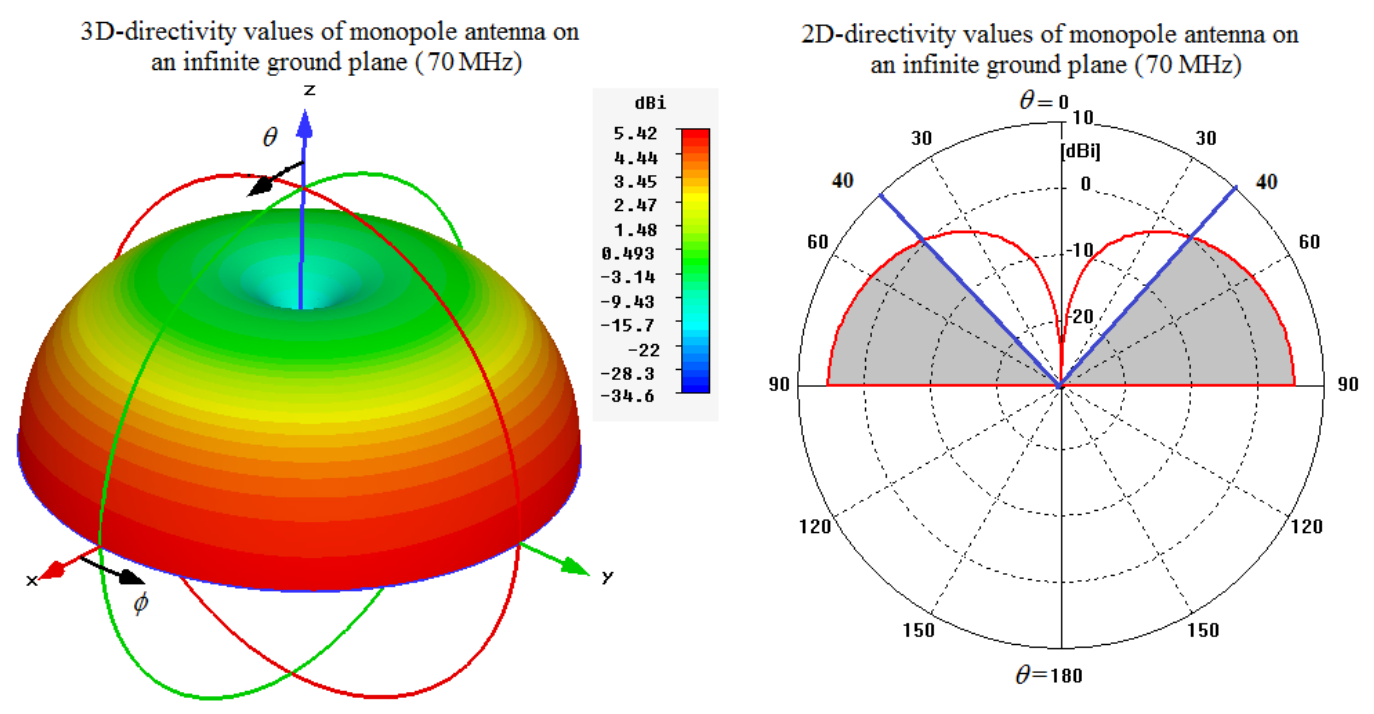

Figure 7.13 Far-field radiation patterns of monopole antenna at $70 \mathrm{MHz}$

Figure 7.14 shows the far-field radiation pattern of VHF upper antenna which is spatially filtered and examined for $40^{\circ} \leq \theta \leq 90^{\circ}$ and $0^{\circ} \leq \phi \leq 360^{\circ}$ and Figure 7.15 shows the far-field radiation pattern of VHF lower antenna which is spatially filtered and examined for $90^{\circ} \leq \theta \leq 140^{\circ}$ and $0^{\circ} \leq \phi \leq 360^{\circ}$. According to these figures:

- $78 \%$ (greater than $50 \%$ ) of the directivity values of VHF upper antenna for $40^{\circ} \leq \theta \leq 90^{\circ}$ and $0^{\circ} \leq \phi \leq 360^{\circ}$ is greater than $0 \mathrm{dBi}$.

- $85 \%$ (greater than $50 \%$ ) of the directivity values of VHF lower antenna for $90^{\circ} \leq \theta \leq 140^{\circ}$ and $0^{\circ} \leq \phi \leq 360^{\circ}$ is greater than $0 \mathrm{dBi}$.

As a result both antennas are considered to have "sufficient coverage" at $70 \mathrm{MHz}$ at their optimal places where the average coupling between them is minimum. 


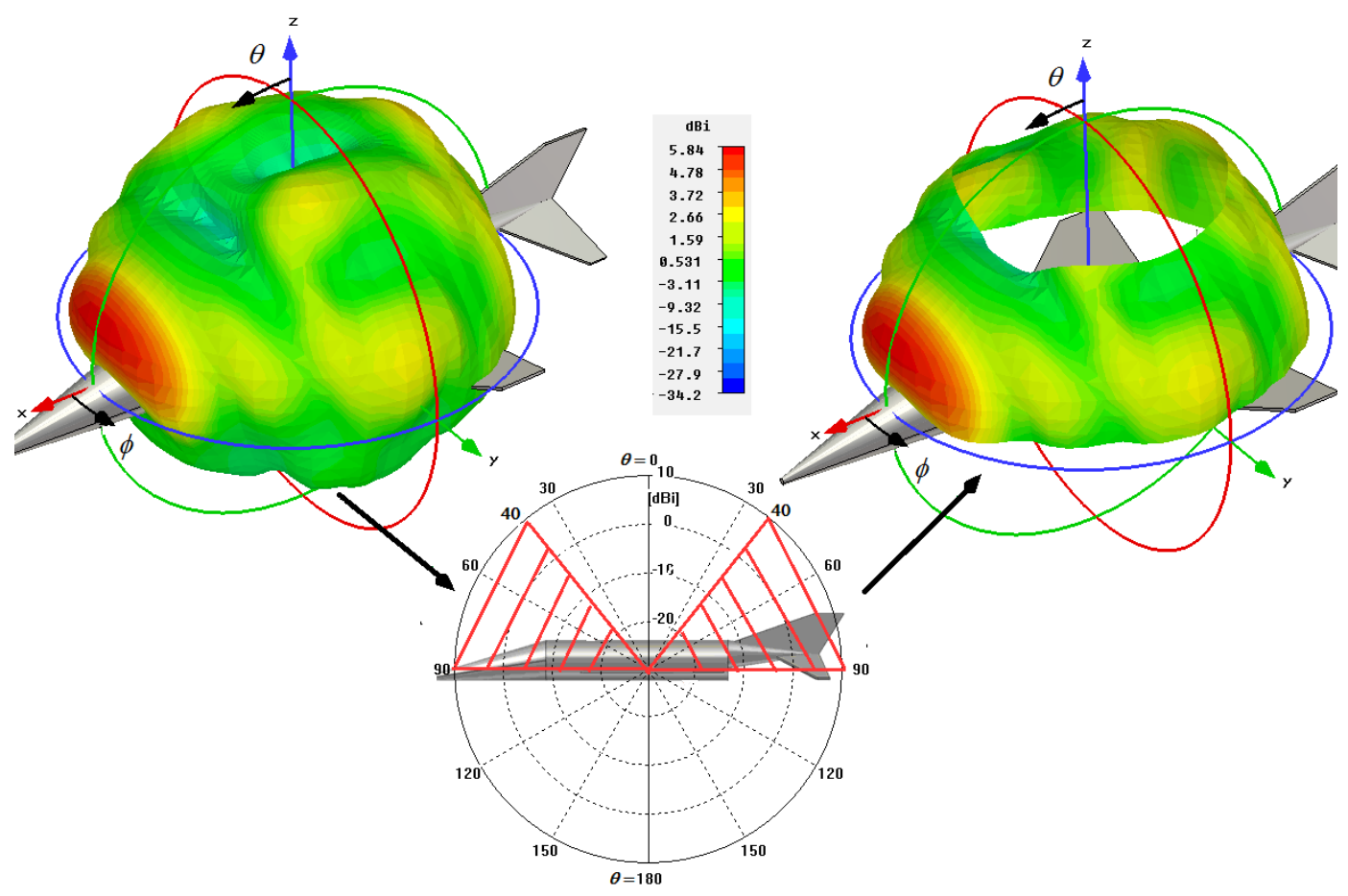

Figure 7.14 Far-field radiation pattern of VHF upper antenna at $70 \mathrm{MHz}$

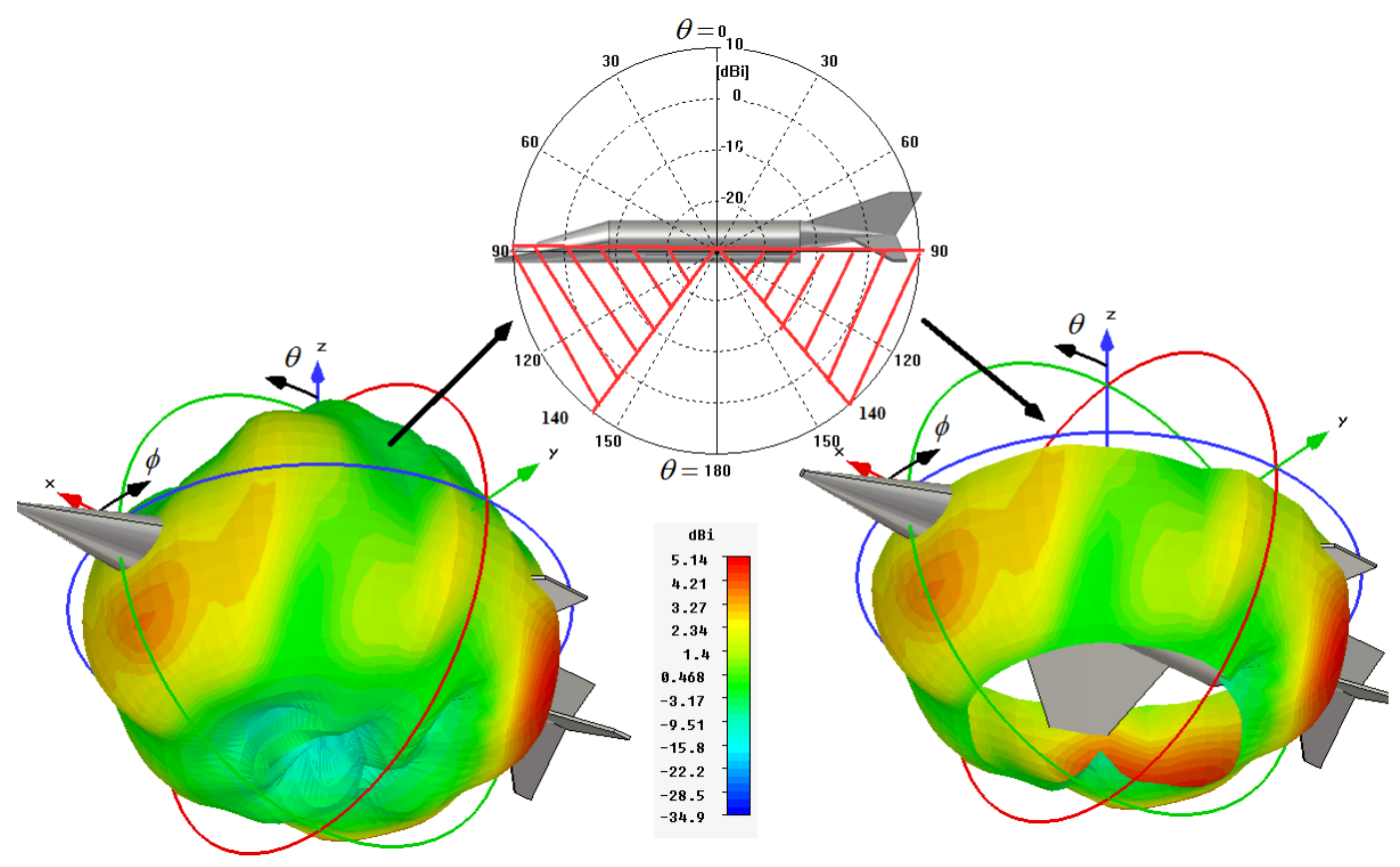

Figure 7.15 Far-field radiation pattern of VHF lower antenna at $70 \mathrm{MHz}$ 


\subsubsection{Far-field Radiation Pattern Analyses for Aircraft Antennas at 80 MHz}

Three-dimensional and two-dimensional far-field radiation patterns of a VHF monopole antenna on an infinite ground plane at $80 \mathrm{MHz}$ are given in Figure 7.16. According to this figure, for $40^{\circ} \leq \theta \leq 90^{\circ}$ (in a range of $50^{\circ}$ ) and $0^{\circ} \leq \phi \leq 360^{\circ}$, directivity of monopole antenna on an infinite ground plane is greater than $0 \mathrm{dBi}$.
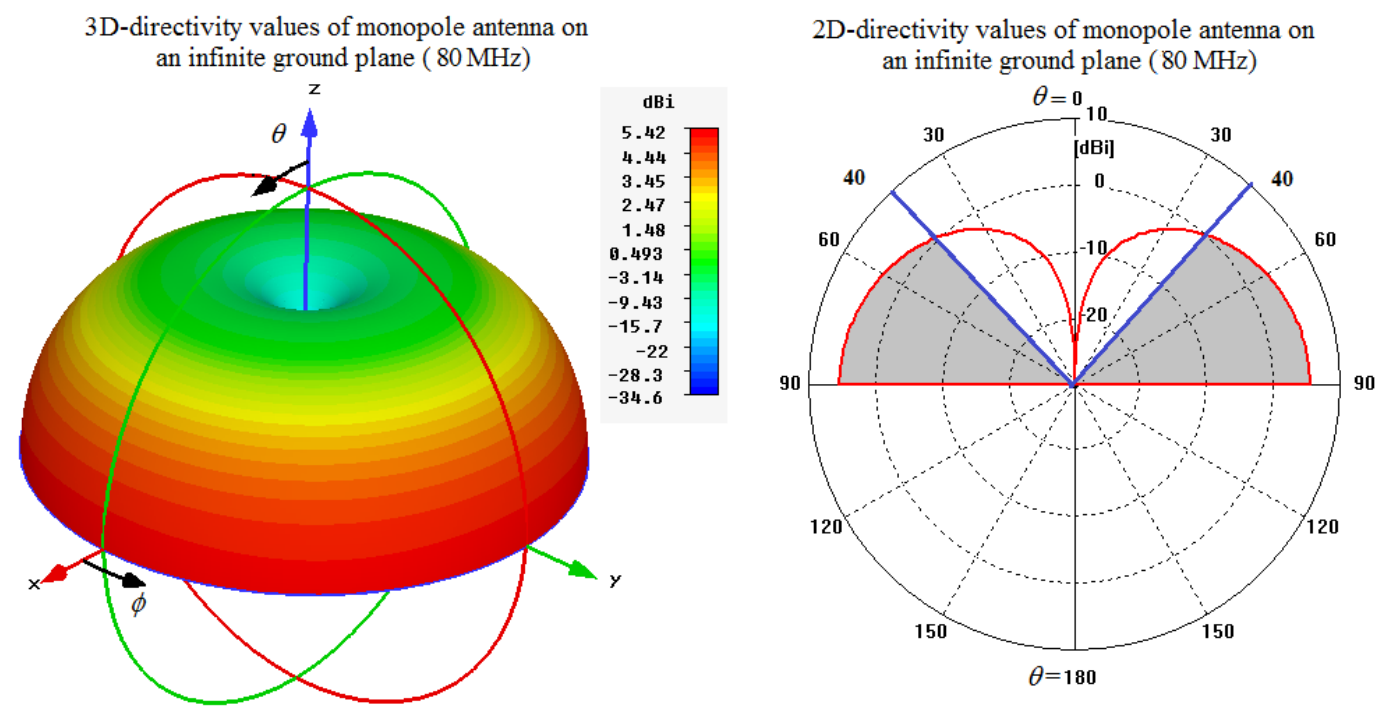

Figure 7.16 Far-field radiation patterns of monopole antenna at $80 \mathrm{MHz}$

Figure 7.17 shows the far-field radiation pattern of VHF upper antenna which is spatially filtered and examined for $40^{\circ} \leq \theta \leq 90^{\circ}$ and $0^{\circ} \leq \phi \leq 360^{\circ}$ and Figure 7.18 shows the far-field radiation pattern of VHF lower antenna which is spatially filtered and examined for $90^{\circ} \leq \theta \leq 140^{\circ}$ and $0^{\circ} \leq \phi \leq 360^{\circ}$. According to these figures: 
- $76 \%$ (greater than $50 \%$ ) of the directivity values of VHF upper antenna for $40^{\circ} \leq \theta \leq 90^{\circ}$ and $0^{\circ} \leq \phi \leq 360^{\circ}$ is greater than $0 \mathrm{dBi}$.

- $84 \%$ (greater than $50 \%$ ) of the directivity values of VHF lower antenna for $90^{\circ} \leq \theta \leq 140^{\circ}$ and $0^{\circ} \leq \phi \leq 360^{\circ}$ is greater than $0 \mathrm{dBi}$.

As a result both antennas are considered to have "sufficient coverage" at $80 \mathrm{MHz}$ at their optimal places where the average coupling between them is minimum.

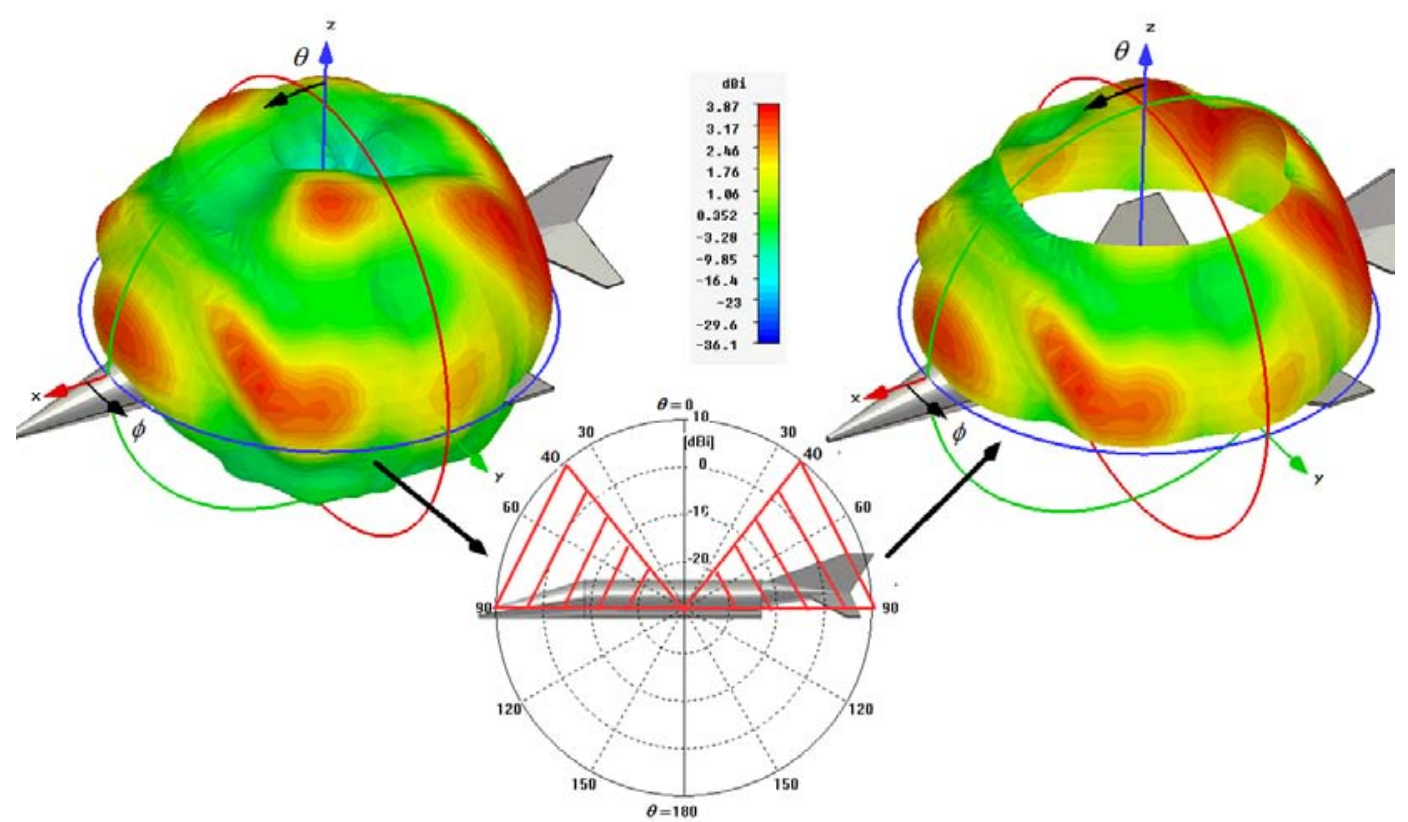

Figure 7.17 Far-field radiation pattern of VHF upper antenna at $80 \mathrm{MHz}$ 


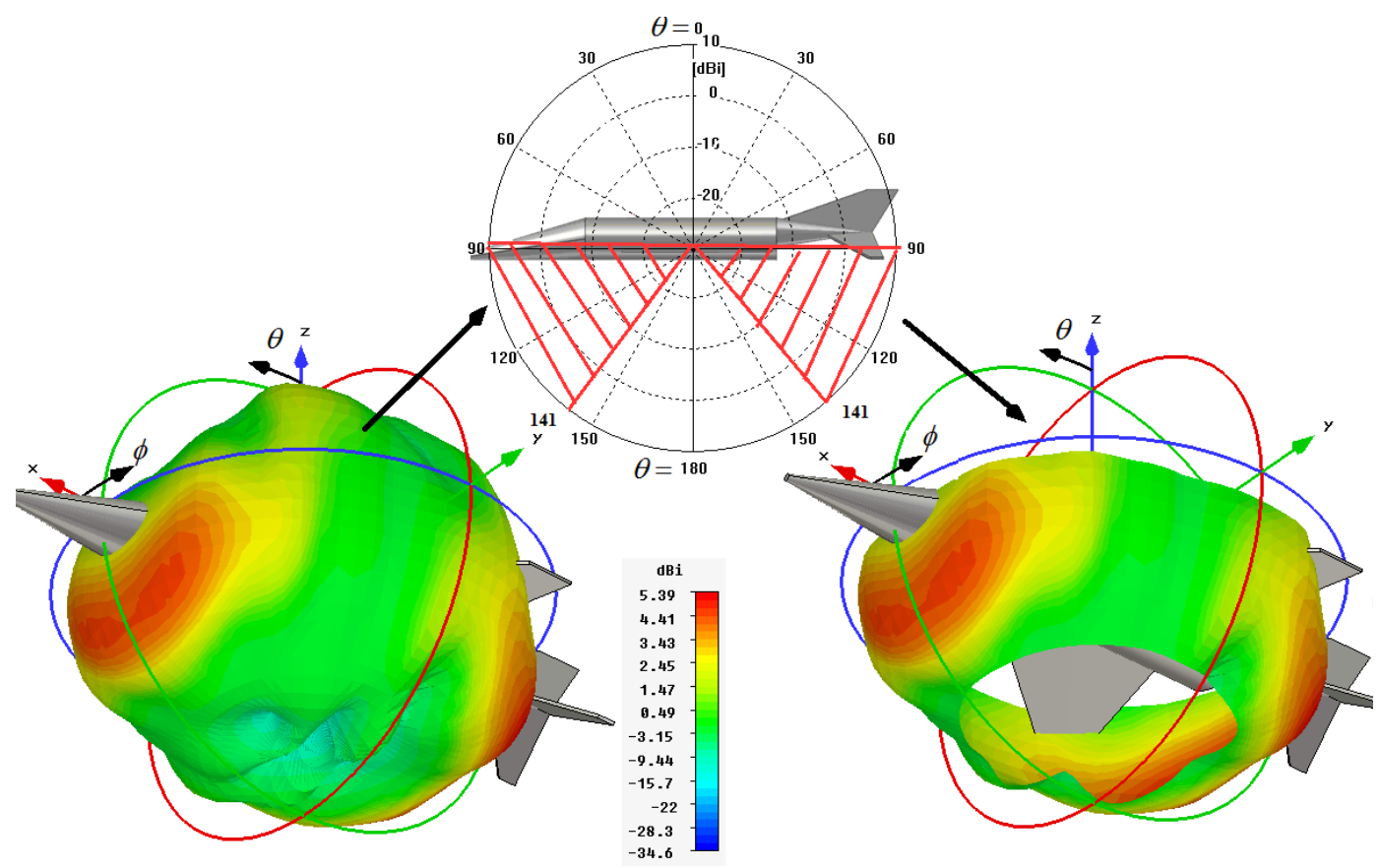

Figure 7.18 Far-field radiation pattern of VHF lower antenna at $80 \mathrm{MHz}$

All of the results of the radiation pattern analyses of aircraft antennas are gathered together and given in Table 7.1.

Table 7.1 Results of the radiation pattern analyses of aircraft antennas

\begin{tabular}{|c|c|c|c|}
\hline $\begin{array}{c}\text { Coordinates } \\
\text { of the } \\
\text { Antennas }\end{array}$ & Frequency & $\begin{array}{c}\text { Percentage of } \\
\text { (Directivity Values } \geq 0 \mathrm{dBi})\end{array}$ & $\begin{array}{c}\text { Angular Ranges } \\
\text { of Interest } \\
\left(0^{\circ} \leq \phi \leq 360^{\circ}\right)\end{array}$ \\
\hline \multirow{4}{*}{$\begin{array}{l}\text { VHF upper } \\
\text { antenna @ } \\
x=0.86 \mathrm{~m}\end{array}$} & $30 \mathrm{MHz}$ & $66 \%$ & $34^{\circ} \leq \theta \leq 90^{\circ}$ \\
\cline { 2 - 4 } & $40 \mathrm{MHz}$ & $72 \%$ & $37^{\circ} \leq \theta \leq 90^{\circ}$ \\
\cline { 2 - 4 } & $50 \mathrm{MHz}$ & $72 \%$ & $37^{\circ} \leq \theta \leq 90^{\circ}$ \\
\cline { 2 - 4 } & $70 \mathrm{MHz}$ & $78 \%$ & $39^{\circ} \leq \theta \leq 90^{\circ}$ \\
\cline { 2 - 4 } & $80 \mathrm{MHz}$ & $78 \%$ & $40^{\circ} \leq \theta \leq 90^{\circ}$ \\
\hline
\end{tabular}


Table 7.1 (continued)

\begin{tabular}{|c|c|c|c|}
\hline $\begin{array}{c}\text { Coordinates } \\
\text { of the } \\
\text { Antennas }\end{array}$ & Frequency & $\begin{array}{c}\text { Percentage of } \\
\text { (Directivity Values } \geq 0 \mathrm{dBi} \text { ) }\end{array}$ & $\begin{array}{c}\text { Angular Ranges } \\
\text { of Interest } \\
\left(0^{\circ} \leq \phi \leq 360^{\circ}\right)\end{array}$ \\
\hline \multirow{4}{*}{$\begin{array}{l}\text { VHF lower } \\
\text { antenna @ } \\
x=7.88 \mathrm{~m}\end{array}$} & $30 \mathrm{MHz}$ & $82 \%$ & $90^{\circ} \leq \theta \leq 146^{\circ}$ \\
\cline { 2 - 4 } & $40 \mathrm{MHz}$ & $79 \%$ & $90^{\circ} \leq \theta \leq 143^{\circ}$ \\
\cline { 2 - 4 } & $60 \mathrm{MHz}$ & $80 \%$ & $90^{\circ} \leq \theta \leq 143^{\circ}$ \\
\cline { 2 - 4 } & $70 \mathrm{MHz}$ & $68 \%$ & $90^{\circ} \leq \theta \leq 141^{\circ}$ \\
\cline { 2 - 4 } & $80 \mathrm{MHz}$ & $85 \%$ & $90^{\circ} \leq \theta \leq 140^{\circ}$ \\
\hline
\end{tabular}

According to Table 7.1, percentages of the directivity values of aircraft antennas within each of the angular ranges and for all frequencies of interest are greater than $50 \%$. Therefore both of the VHF upper and lower antennas are considered to have "sufficient coverage" at the upper and lower hemisphere respectively.

\subsection{Far-field Radiation Pattern Performances of Ship Antennas}

The optimal antenna placement yielding minimum average coupling between the ship antennas is determined by Particle Swarm Optimization. In this optimization the initial population is generated by Latin hypercube distribution technique. In the optimal placement, HF fore antenna is located at $x=25.1 \mathrm{~m}$ and $y=3.4 \mathrm{~m}$, and HF aft antenna is located at $x=1.5 \mathrm{~m}$ and $y=1.2 \mathrm{~m}$.

In this antenna placement, both HF fore and aft antennas radiate/receive in the upper hemisphere. In order to analyze the total coverage of the antennas on the upper hemisphere, contribution of both antennas is considered. Therefore, two HF antennas are assumed to operate complementarily in terms of directivity in upper hemisphere. While determining "resultant directivity” for a particular direction on the upper hemisphere, the directivity value of the antenna which is higher than 
that of the other antenna is chosen. For example; for a particular direction on the upper hemisphere, if directivity value of HF fore antenna is higher than that of HF aft antenna, then directivity value of HF fore antenna is assigned for that particular direction.

The "resultant directivities" of ship antennas are compared with directivities of monopole antenna on an infinite ground plane as follows:

- The directivities of the ship antennas are calculated at $10 \mathrm{MHz}, 20 \mathrm{MHz}$ and $30 \mathrm{MHz}$.

- Directivities of an HF monopole antenna on an "infinite ground plane", having the same electrical and physical properties with ship antennas are calculated at same distinct frequencies.

- At each frequency, "the ranges of azimuth and elevation angles ( $\phi$ and $\theta$ )” in where the directivity of the monopole antenna on an infinite gorund plane is greater than $0 \mathrm{dBi}$, is determined.

- At each frequency, "resultant directivity" of ship antennas is examined within the angular ranges mentioned in the bullet above. If minimum of 50 $\%$ of "resultant directivity” values of ship antennas within these ranges are greater than $0 \mathrm{dBi}$, then those antennas are assumed to have "sufficient coverage”, which means, those antennas radiate/receive sufficiently.

\subsubsection{Far-field Radiation Pattern Analyses for Ship Antennas at $10 \mathrm{MHz}$}

Three-dimensional and two-dimensional far-field radiation patterns of an HF monopole antenna on an infinite ground plane at $10 \mathrm{MHz}$ are given in Figure 7.19. 
According to this figure, for $40^{\circ} \leq \theta \leq 90^{\circ}$ and $0^{\circ} \leq \phi \leq 360^{\circ}$, directivity of monopole antenna on an infinite ground plane is greater than $0 \mathrm{dBi}$.
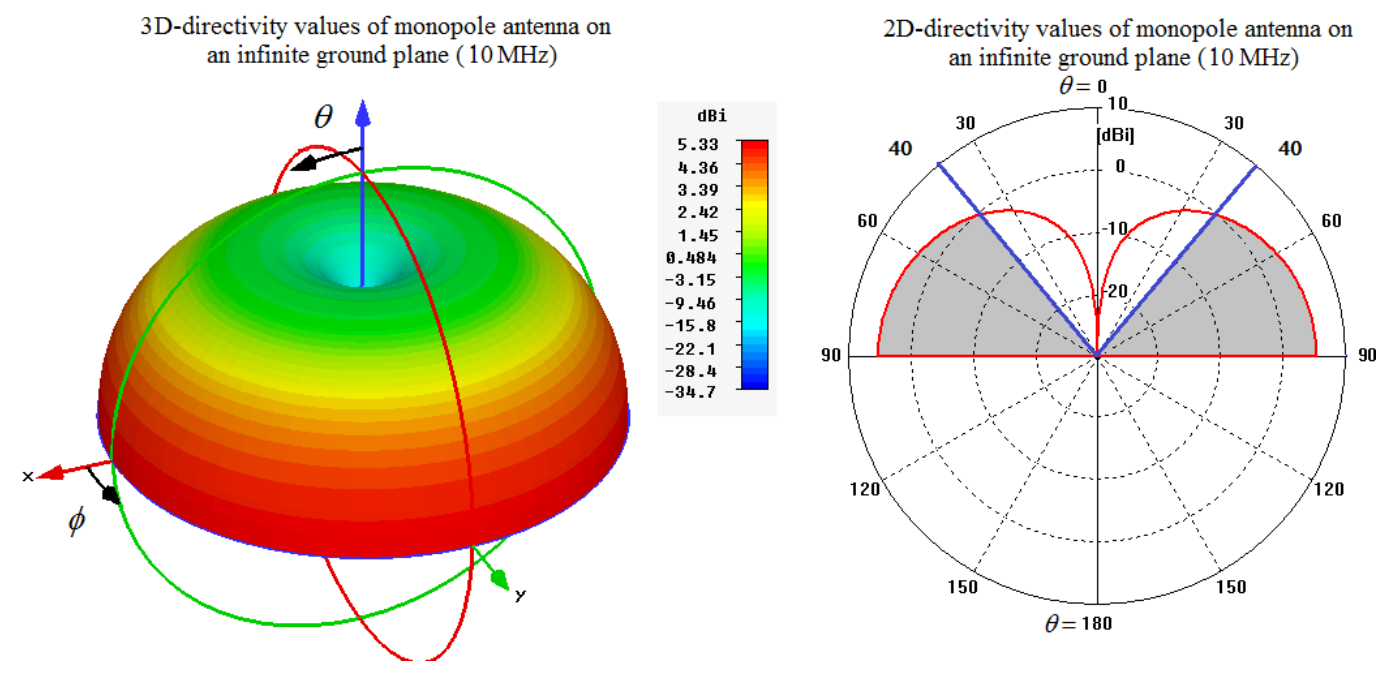

Figure 7.19 Far-field radiation patterns of monopole antenna at $10 \mathrm{MHz}$

Figure 7.20 shows the far-field radiation patterns of HF fore and aft antennas which are spatially filtered and examined for $40^{\circ} \leq \theta \leq 90^{\circ}$ and $0^{\circ} \leq \phi \leq 360^{\circ}$. According to these radiation patterns, "resultant directivity" is calculated as explained above, for $40^{\circ} \leq \theta \leq 90^{\circ}$ and $0^{\circ} \leq \phi \leq 360^{\circ}$. According to this calculation:

- $88 \%$ (greater than $50 \%$ ) of the "resultant directivity" values of HF fore and aft antennas for $40^{\circ} \leq \theta \leq 90^{\circ}$ and $0^{\circ} \leq \phi \leq 360^{\circ}$ are greater than 0 $\mathrm{dBi}$.

As a result; both antennas are considered to have "sufficient coverage" at $10 \mathrm{MHz}$ at their optimal places where the average coupling between them is minimum. 


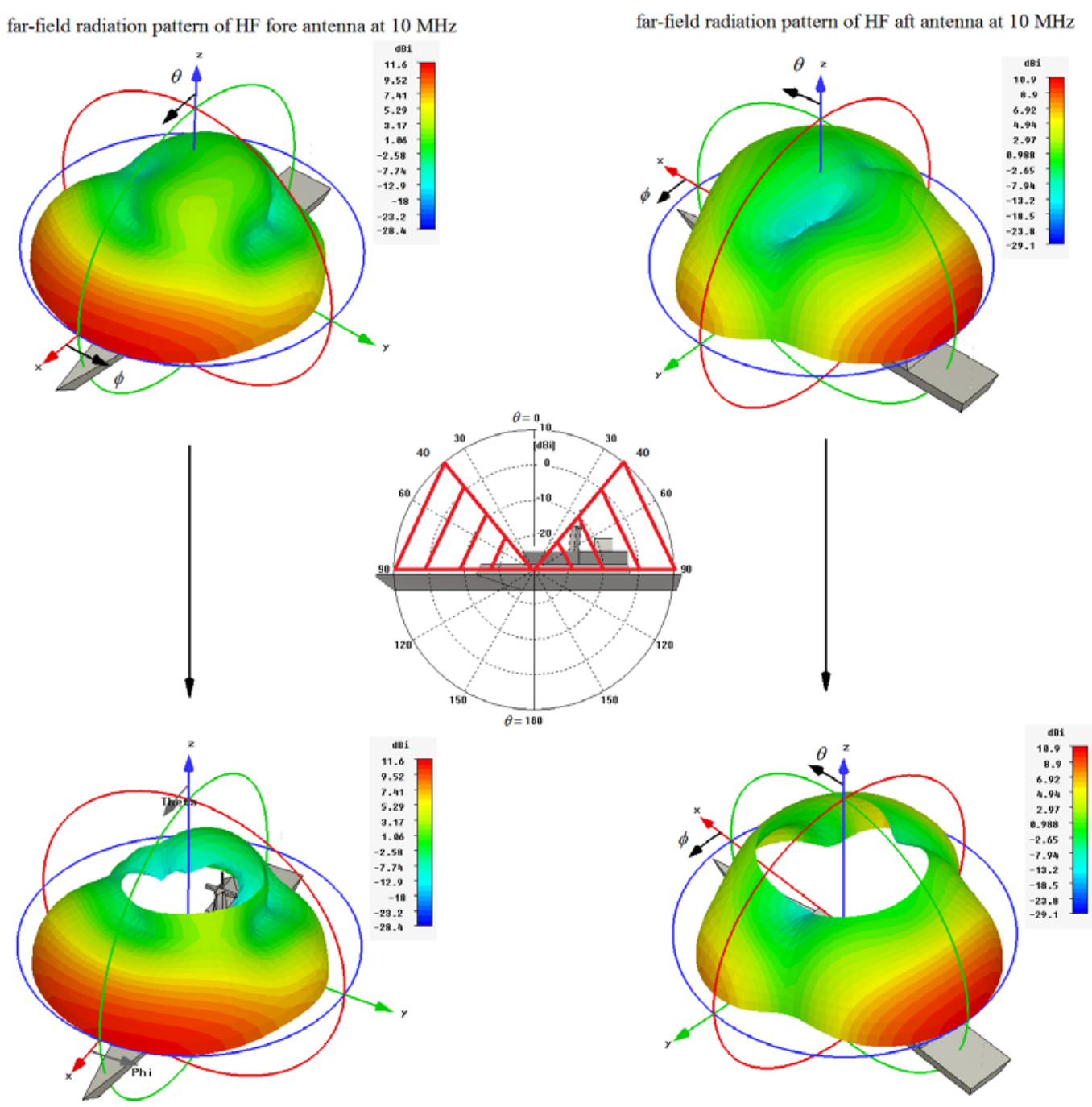

Figure 7.20 Far-field radiation patterns of HF fore and aft antennas at $10 \mathrm{MHz}$

\subsubsection{Far-field Radiation Pattern Analyses for Ship Antennas at $20 \mathrm{MHz}$}

Three-dimensional and two-dimensional far-field radiation patterns of an HF monopole antenna on an infinite ground plane at $20 \mathrm{MHz}$ are given in Figure 7.21. 
According to this figure, for $60^{\circ} \leq \theta \leq 90^{\circ}$ and $0^{\circ} \leq \phi \leq 360^{\circ}$, directivity of monopole antenna on an infinite ground plane is greater than $0 \mathrm{dBi}$.
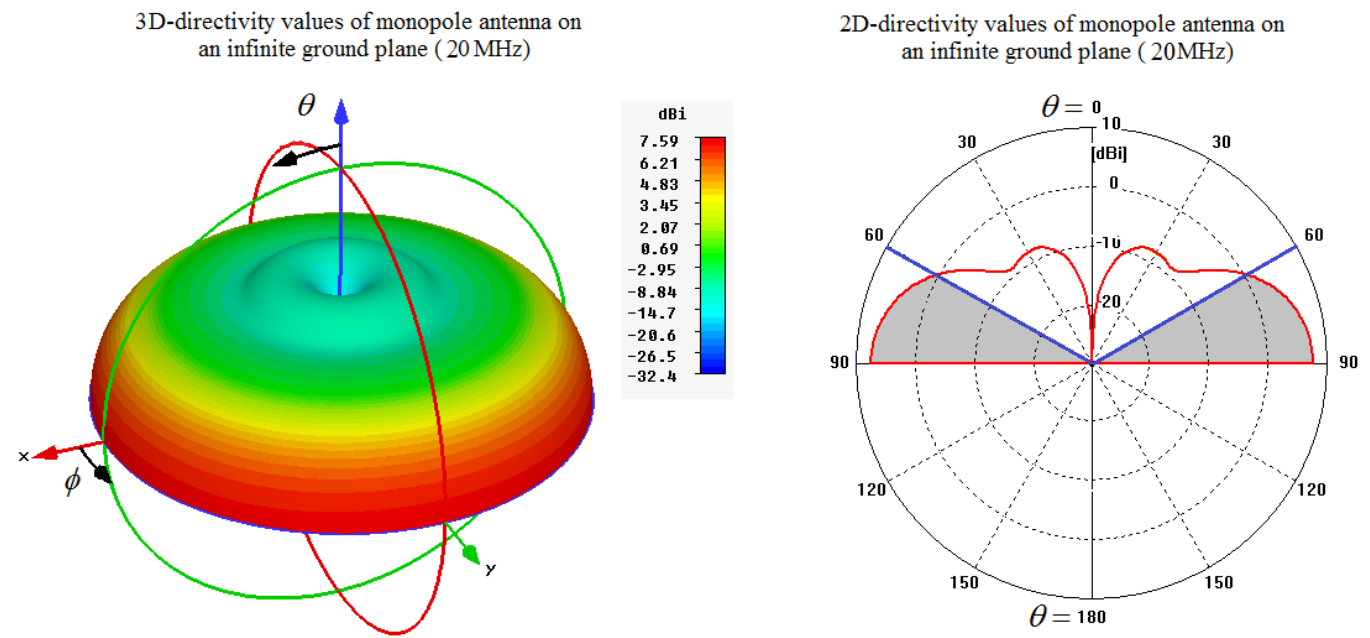

Figure 7.21 Far-field radiation patterns of monopole antenna at $20 \mathrm{MHz}$

Figure 7.22 shows the far-field radiation patterns of HF fore and aft antennas which are spatially filtered and examined for $60^{\circ} \leq \theta \leq 90^{\circ}$ and $0^{\circ} \leq \phi \leq 360^{\circ}$. According to these radiation patterns, "resultant directivity" is calculated as explained above, for $60^{\circ} \leq \theta \leq 90^{\circ}$ and $0^{\circ} \leq \phi \leq 360^{\circ}$. According to this calculation:

- $89 \%$ (greater than $50 \%$ ) of the "resultant directivity" values of HF fore and aft antennas for $60^{\circ} \leq \theta \leq 90^{\circ}$ and $0^{\circ} \leq \phi \leq 360^{\circ}$ are greater than 0 $\mathrm{dBi}$.

As a result; both antennas are considered to have "sufficient coverage" at $20 \mathrm{MHz}$ at their optimal places where the average coupling between them is minimum. 


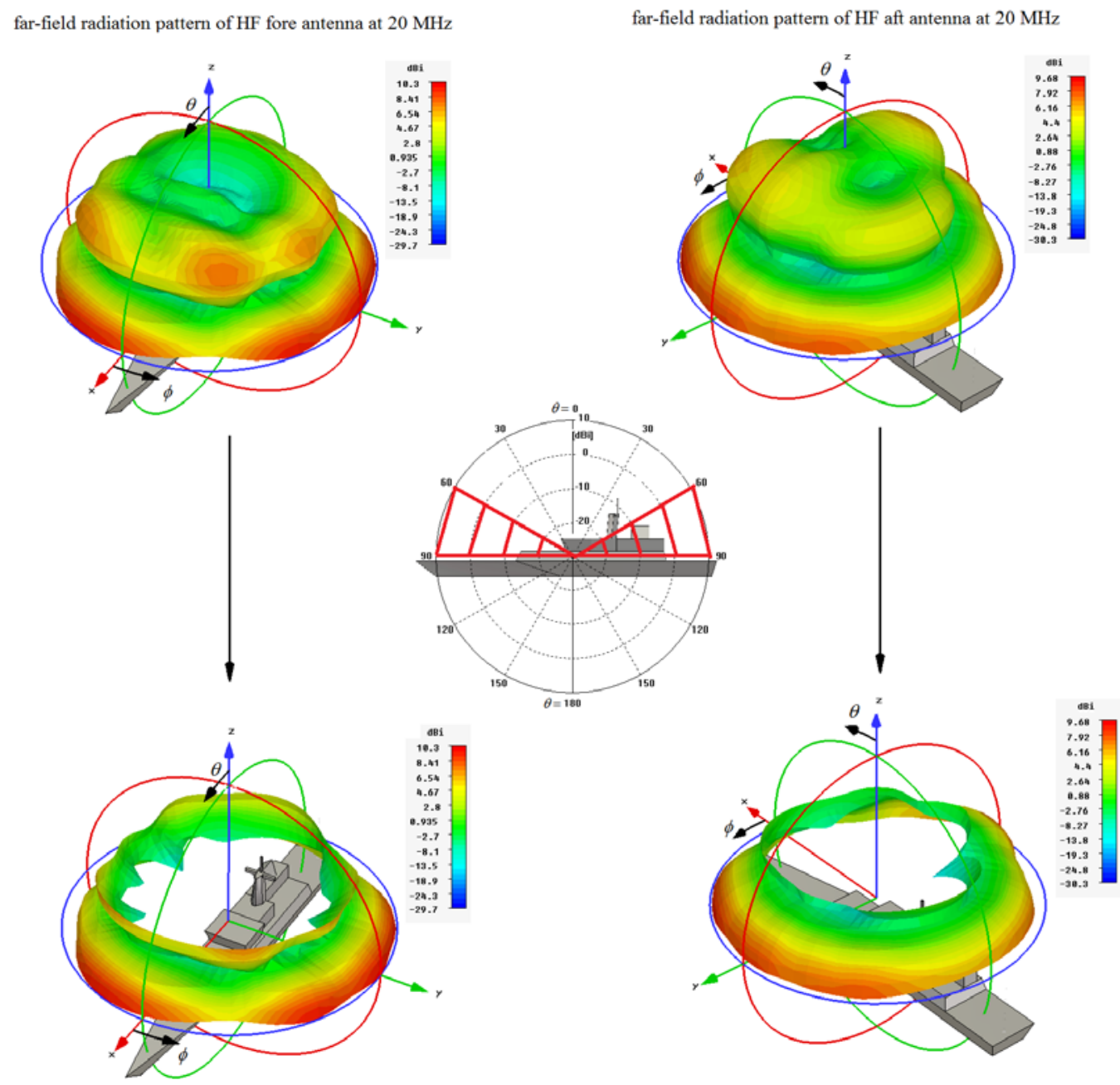

Figure 7.22 Far-field radiation patterns of HF fore and aft antennas at $20 \mathrm{MHz}$

\subsubsection{Far-field Radiation Pattern Analyses for Ship Antennas at $30 \mathrm{MHz}$}

Three-dimensional and two-dimensional far-field radiation patterns of an HF monopole antenna on an infinite ground plane at $30 \mathrm{MHz}$ are given in Figure 7.23 According to this figure, for $22^{\circ} \leq \theta \leq 70^{\circ}$ and $0^{\circ} \leq \phi \leq 360^{\circ}$, directivity of monopole antenna on an infinite ground plane is greater than $0 \mathrm{dBi}$. 


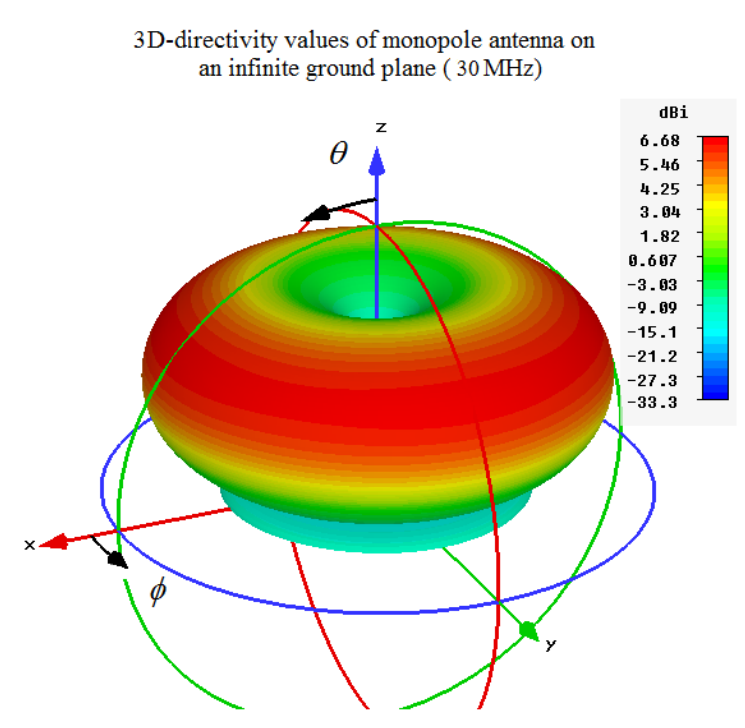

2D-directivity values of monopole antenna on an infinite ground plane $(30 \mathrm{MHz})$

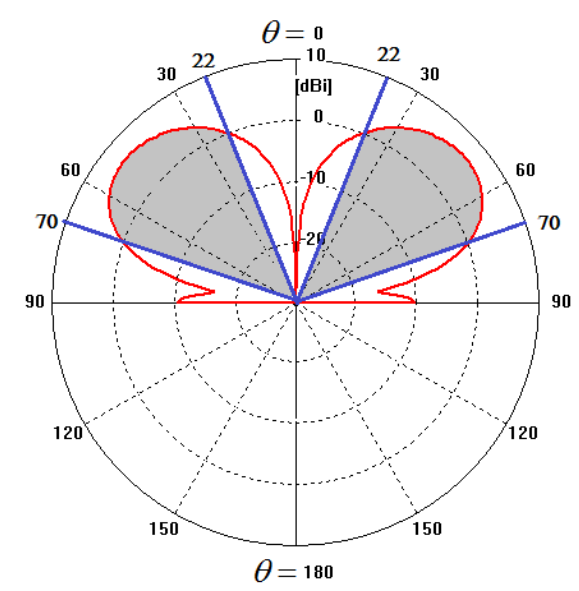

Figure 7.23 Far-field radiation patterns of monopole antenna at $30 \mathrm{MHz}$

Figure 7.24 shows the far-field radiation patterns of HF fore and aft antennas which is spatially filtered and examined for $22^{\circ} \leq \theta \leq 70^{\circ}$ and $0^{\circ} \leq \phi \leq 360^{\circ}$. According to these radiation patterns, "resultant directivity" is calculated as explained above, for $22^{\circ} \leq \theta \leq 70^{\circ}$ and $0^{\circ} \leq \phi \leq 360^{\circ}$. According to this calculation:

- $99 \%$ (greater than $50 \%$ ) of the "resultant directivity" values of HF fore and aft antennas for $22^{\circ} \leq \theta \leq 70^{\circ}$ and $0^{\circ} \leq \phi \leq 360^{\circ}$ are greater than $0 \mathrm{dBi}$.

As a result both antennas are considered to have "sufficient coverage" at $30 \mathrm{MHz}$ at their optimal places where the average coupling between them is minimum. 


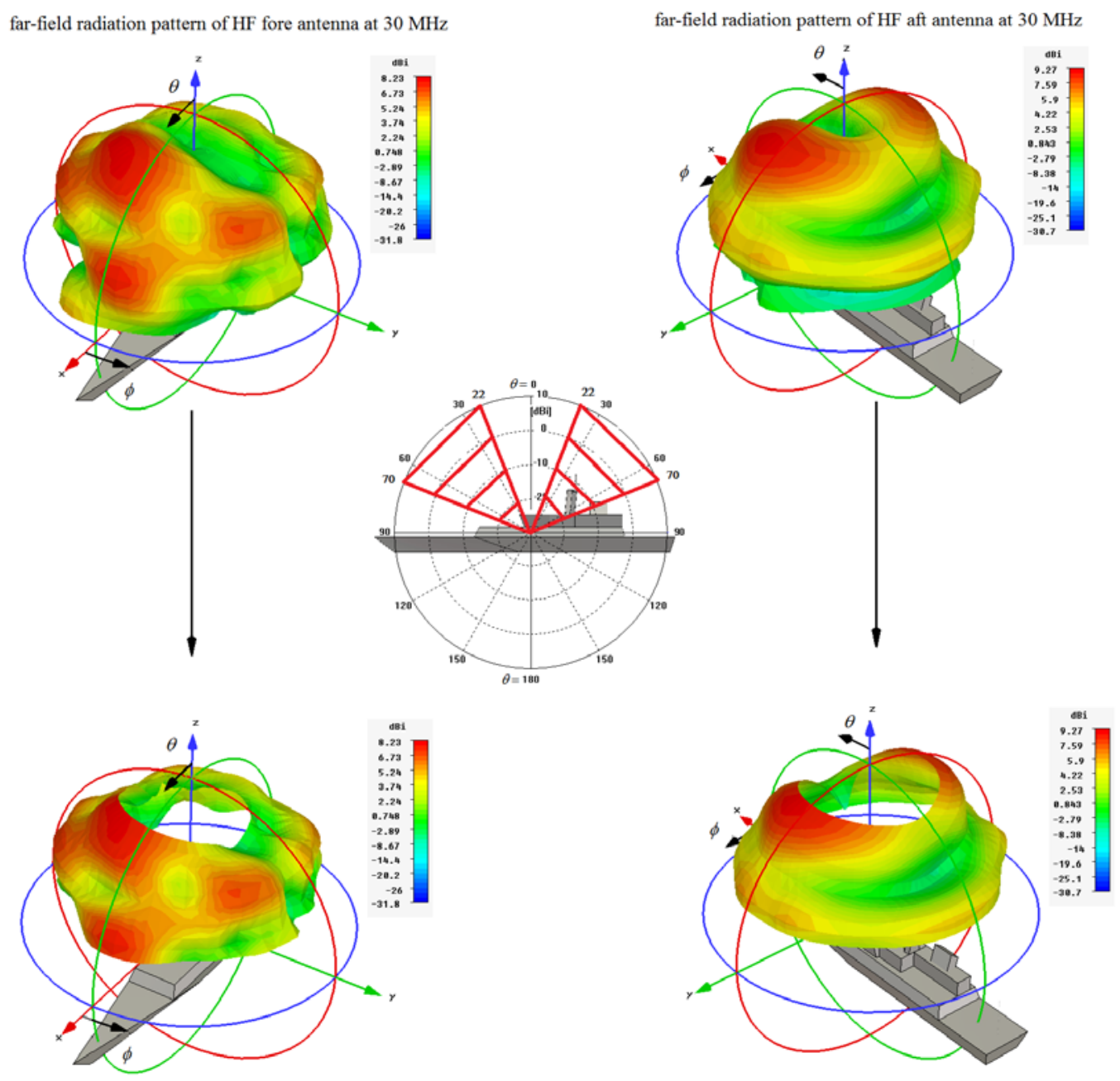

Figure 7.24 Far-field radiation patterns of $\mathrm{HF}$ fore and aft antennas at $30 \mathrm{MHz}$

Results of the radiation pattern analyses of ship antennas are gathered and given in Table 7.2. 
Table 7.2 Results of the radiation pattern analyses of ship antennas

\begin{tabular}{|c|c|c|c|}
\hline $\begin{array}{l}\text { Coordinates } \\
\text { of the } \\
\text { Antennas }\end{array}$ & Frequency & $\begin{array}{c}\text { Percentage of } \\
\text { ("Resultant Directivity" } \\
\text { Values } \geq 0 \mathrm{dBi} \text { ) }\end{array}$ & $\begin{array}{c}\text { Angular Ranges } \\
\text { of Interest } \\
\left(0^{\circ} \leq \phi \leq 360^{\circ}\right) \\
\end{array}$ \\
\hline $\begin{array}{c}\text { HF fore } \\
\text { antenna @ } \\
x=25.1 \mathrm{~m}\end{array}$ & $10 \mathrm{MHz}$ & $88 \%$ & $40^{\circ} \leq \theta \leq 90^{\circ}$ \\
\hline \& & $20 \mathrm{MHz}$ & $89 \%$ & $60^{\circ} \leq \theta \leq 90^{\circ}$ \\
\hline $\begin{array}{c}\text { HF aft } \\
\text { antenna @ } \\
x=1.5 \mathrm{~m} \\
y=1.2 \mathrm{~m}\end{array}$ & $30 \mathrm{MHz}$ & $99 \%$ & $22^{\circ} \leq \theta \leq 70^{\circ}$ \\
\hline
\end{tabular}

According to Table 7.2, percentages of the "resultant directivity" values of ship antennas for each of the angular ranges and for all frequencies of interest are greater than $50 \%$. Therefore both of the HF fore and aft antennas are considered to have "sufficient coverage" in the upper hemisphere. 


\section{CHAPTER 8}

\section{CONCLUSIONS AND FUTURE WORK}

Since the detailed conclusions/discussions about the analyses are already included in the previous chapters, the general conclusions drawn from this thesis work are summarized as follows:

- Optimal antenna placements yielding minimum average coupling between the antennas of two VHF radios on an aircraft platform and between the antennas of two HF radios on a ship platform are determined by genetic algorithm optimization and particle swarm optimization techniques.

- For both platforms, optimal antenna placements and resultant average coupling values determined by two different optimization techniques are found to be very close to each other. The results and performances of two global optimization techniques are given in detail and compared with each other in the related sections above

- For both platforms, each antenna placement optimization by genetic algorithm lasts for about one day and each antenna placement optimization by particle swarm optimization lasts for about two days. Both time spans are "real-time" values and are found to be tolerable.

- For each platform, far-field radiation pattern performances of the antennas at their optimal places are analyzed in terms of directivity and coverage. 
As a result, radiation performances (coverage) of the antennas for both platforms are found to be sufficient.

Further studies which can improve or extend the achievements of this thesis work are summarized as follows:

- For each platform, similar coupling optimizations and far-field performance analyses can be performed for different antenna lengths and for different frequency bands of operation.

- In this thesis work the, RF components like impedance matching units, pre-selector filters, etc. which are used for improving the performances of the antennas and protective components like radomes are not considered in the simulations. For more realistic results, these components can be added to the antenna models and considered in the simulations.

- For each platform, similar coupling optimizations can be performed with different genetic algorithm and particle swarm optimization parameters.

- In this thesis work, coupling between the antennas are minimized for the whole frequency band of operation for each platform. Instead of this approach, maximum value of the coupling or the coupling for at a particular frequency of interest can be minimized for each platform.

- Optimal antenna placements can further be improved by using local optimization techniques like the Quasi Newton, the Powell and the NelderMead Simplex optimization techniques. 


\section{REFERENCES}

[1] Koper E. M., Wood W. D. and Schneider S. W., “Aircraft Antenna Coupling Minimization Using Genetic Algorithms and Approximations”, IEEE Transactions on Aerospace and Electronic Systems, Vol. 40, No. 2, pp. 742-751, April 2004

[2] Koper E. M., Wood W. D. and Schneider S. W., “Aircraft Antenna Coupling Minimization Using Genetic Algorithms and Approximations”, IEEE Transactions on Aerospace and Electronic Systems, Vol. 40, No. 2, pp. 742-751, April 2004

[3] Aydemir, M. E., Günel, T. and Üstüner, F., "Genetic approach to the minimization of the coupling between aircraft antennas”, International Journal of Electronics and Communication, (AEÜ), Vol. 60, pp. 299-305, 2006

[4] Suh, T. I., Lee, S. S. and Kim, H. T., Mutual Coupling Reduction of Antennas on a Complex Superstructure, Journal of Electromagnetic Waves and Applications, Vol. 18, No. 7, pp. 983-991, 2004

[5] Barney, M. J., Knapil, J. M. and Haupt, R. L., "Determining an optimal antenna placement using a genetic algorithm”, Antennas and Propagation Society International Symposium, APSURSI '09, pp. 1-4, 2009

[6] Sivanandam, S. N. and Deepa, S. N., Introduction to Genetic Algorithms, Springer-Verlag, Berlin, Heidelberg, 2008 
[7] Michiels, W., Aarts, E. and Korst, J., Theoretical Aspects of Local Search, Springer-Verlag, Berlin, Heidelberg, 2007

[8] “CST Microwave Studio, Optimizer-Specials”, CST Studio Suite Help 2009, 2009

[9] Nelder, J. A. and Mead, R., “A Simplex Method for Function Minimization”, Computer Journal, Vol. 7, pp. 308-313, 1965

[10] Blasone, R. S., Madsen, H. and Rosbjerg, D., "Parameter estimation in distributed hydrological modeling: comparison of global and local optimization techniques”, Nordic Hydrology, Vol. 38, No. 4-5, pp. 451476, IWA Publishing, 2007

[11] Haupt, R. L. and Haupt, S. E., Practical Genetic Algorithms, $2^{\text {nd }}$ edition, Wiley \& Sons, New Jersey, 2004

[12] Holland, J. H., Adaptation in Natural and Artificial Systems, University of Michigan Press, Ann Arbor, 1975

[13] Goldberg, D. E., “Computer-aided gas pipeline operation using genetic algorithms and rule learning”, PhD Thesis, University of Michigan, Ann Arbor, 1989

[14] “Using the Genetic Algorithm”, Mathworks - MATLAB Help [online], http://www.mathworks.com/access/helpdesk/help/toolbox/gads/f6010dfi3. html, date of last access June 26, 2010 
[15] Weise, T., Global Optimization Algorithms, Theory and Application, Version: 2009-06-26 [online], http://www.it-weise.de/, date of last access July 4, 2010

[16] McKay, M.D., Beckman, R.J. and Conover, W.J., “A Comparison of Three Methods for Selecting Values of Input Variables in the Analysis of Output from a Computer Code", Technometrics (American Statistical Association), Vol. 21, No. 2, pp. 239-245, May, 1979

[17] Rahmat-Samii, Y. and Michielssen, E., Electromagnetic Optimization by Genetic Algorithm, Wiley \& Sons, New York, 1999

[18] Kennedy, J. and Eberhart, R., "Particle Swarm Optimization”, Proceedings of IEEE International Conference on Neural Networks, IV, pp. 1942-1948, 1995

[19] Eberhart, R. C., and Kennedy, J., “A new optimizer using particle swarm theory”, Proceedings of the Sixth International Symposium on Micro Machine and Human Science, pp. 39-43, 1995

[20] Poli, R., Kennedy, J. and Blackwell, T., Particle swarm optimization-An overview, Springer Science + Business Media, LLC, 2007

[21] Qinghai, B., “Analysis of Particle Swarm Optimization Algorithm”, Computer and Information Science, Vol. 3, No.1, pp. 180-184 February, 2010 
[22] Shi, Y. and Eberhart, R. C., “A Modified Particle Swarm Optimizer”, Proceedings of the IEEE International Conference on Evolutionary Computation, pp. 69-73, May 4-9, 1998

[23] Das, S., Abraham, A. and Konar A., "Particle Swarm Optimization and Differential Evolution Algorithms: Technical Analysis, Applications and Hybridization Perspectives”, Studies in Computational Intelligence (SCI) 116, pp. 1-38, Springer-Verlag Berlin Heidelberg, 2008

[24] Xu, S. and Rahmat-Samii, Y., "Boundary Conditions in Particle Swarm Optimization Revisited”, IEEE Transactions on Antennas and Propagation, Vol. 55, No. 3, March 2007

[25] CST - Corporate [online], www.cst.com, date of last access September 26, 2010

[26] “CST MWS - Workflow \& Solver Overview”, CST Studio Suite Help 2009, 2009

[27] Weiland, T., “A discretization method for the solution of Maxwell's equations for sixcomponent fields”, International Journal of Electronics and Communication, (AEÜ), Vol. 31, pp. 116-120, 1977.

[28] “Advanced Topics Tutorial, Performance Improvements”, CST Studio Suite ${ }^{\mathrm{TM}}$ Help 2006, 2006

[29] “Getting Started Tutorial, Choose the Right Port”, CST Studio Suite 2006, pp.119-120, 2005 
[30] Davidson, A. S. and Thiele, A. G., "A Hybrid Method of Moments-GTD Technique for Computing Electromagnetic Coupling Between Two Monopole Antennas on a Large Cylindrical Surface”, IEEE Transactions on Electromagnetic Compatibility, Vol. EMC-26, No. 2, pp. 90-96, May 1984 